\title{
High-order accurate entropy stable adaptive moving mesh finite difference schemes for special relativistic (magneto)hydrodynamics
}

\author{
Junming Duan, Huazhong Tang* \\ Center for Applied Physics and Technology, HEDPS and LMAM, School of Mathematical Sciences, Peking \\ University, Beijing 100871, P.R. China
}

\begin{abstract}
This paper develops high-order accurate entropy stable (ES) adaptive moving mesh finite difference schemes for the two- and three-dimensional special relativistic hydrodynamic (RHD) and magnetohydrodynamic (RMHD) equations, which is the high-order accurate extension of [J.M. Duan and H.Z. Tang, Entropy stable adaptive moving mesh schemes for 2D and 3D special relativistic hydrodynamics, J. Comput. Phys., 426(2021), 109949]. The key point is the derivation of the higher-order accurate entropy conservative (EC) and ES finite difference schemes in the curvilinear coordinates by carefully dealing with the discretization of the temporal and spatial metrics and the Jacobian of the coordinate transformation and constructing the high-order EC and ES fluxes with the discrete metrics. The spatial derivatives in the source terms of the symmetrizable RMHD equations and the geometric conservation laws are discretized by using the linear combinations of the corresponding second-order case to obtain high-order accuracy. Based on the proposed high-order accurate EC schemes and the high-order accurate dissipation terms built on the WENO reconstruction, the high-order accurate ES schemes are obtained for the RHD and RMHD equations in the curvilinear coordinates. The mesh iteration redistribution or adaptive moving mesh strategy is built on the minimization of the mesh adaption functional. Several numerical tests are conducted to validate the shock-capturing ability and high efficiency of our high-order accurate ES adaptive moving mesh methods on the parallel computer system with the MPI communication. The numerical results show that the high-order accurate ES adaptive moving mesh schemes outperform both their counterparts on the uniform mesh and the second-order ES adaptive moving mesh schemes. Keywords: High-order accuracy, entropy stable scheme, adaptive moving mesh, relativistic hydrodynamics (RHD), relativistic magnetohydrodynamics (RMHD)
\end{abstract}

\footnotetext{
${ }^{*}$ Corresponding author. Fax: +86-10-62751801.

Email addresses: duanjm@pku.edu.cn (Junming Duan), hztang@math.pku.edu.cn (Huazhong Tang)
} 


\section{Introduction}

This paper is concerned with the high-order accurate numerical schemes for the special relativistic hydrodynamic (RHD) and magnetohydrodynamic (RMHD) equations, which consider the relativistic description for the dynamics of the fluid (gas) at nearly the speed of light when the astrophysical phenomena are investigated from stellar to galactic scales, e.g. the core collapse supernovae, the coalescing neutron stars, the active galactic nuclei, the formation of black holes, the gamma-ray bursts, and the superluminal jets etc. In the covariant form, the four-dimensional space-time RMHD equations can be written as follows [1]

$$
\partial_{\alpha}\left(\rho u^{\alpha}\right)=0, \partial_{\alpha}\left(T^{\alpha \beta}\right)=0, \partial_{\alpha}\left(\Psi^{\alpha \beta}\right)=0
$$

where the Einstein summation convention has been used, $\rho$ and $u^{\alpha}$ denote the rest-mass density and the four-velocity vector, respectively, $\partial_{\alpha}$ denotes the covariant derivative operator with respect to the four-dimensional space-time coordinates $\left(t, x^{1}, x^{2}, x^{3}\right)$, the Greek indices $\alpha, \beta$ run from 0 to 3. In (1.1), the tensor $\Psi^{\alpha \beta}$ can be expressed by $u^{\alpha}$ and four-dimensional magnetic field $b^{\alpha}$ as

$$
\Psi^{\alpha \beta}=u^{\alpha} b^{\beta}-u^{\beta} b^{\alpha},
$$

and the energy-momentum tensor $\mathrm{T}^{\alpha \beta}$ can be decomposed into the fluid part $\mathrm{T}_{f}^{\alpha \beta}$ and the electromagnetic part $\mathrm{T}_{m}^{\alpha \beta}$, defined by

$$
\begin{aligned}
& \mathrm{T}_{f}^{\alpha \beta}=\rho h u^{\alpha} u^{\beta}+p g^{\alpha \beta}, \\
& \mathrm{T}_{m}^{\alpha \beta}=|b|^{2}\left(u^{\alpha} u^{\beta}+g^{\alpha \beta} / 2\right)-b^{\alpha} b^{\beta},
\end{aligned}
$$

where $p$ and $h=1+e+p / \rho$ are respectively the pressure and specific enthalpy, with $e$ the specific internal energy. Throughout this paper, the metric tensor $g^{\alpha \beta}$ is taken as the Minkowski tensor, i.e. $g^{\alpha \beta}= \pm \operatorname{diag}\{-1,1,1,1\}$, and units in which the speed of light is equal to one will be used. The relations between the four-vectors $u^{\alpha}$ and $b^{\alpha}$ and the spatial components of the velocity $\boldsymbol{v}=\left(v_{1}, v_{2}, v_{3}\right)$ and the laboratory magnetic field $\boldsymbol{B}=\left(B_{1}, B_{2}, B_{3}\right)$ are

$$
\begin{aligned}
u^{\alpha} & =W(1, \boldsymbol{v}), \\
b^{\alpha} & =W\left(\boldsymbol{v} \cdot \boldsymbol{B}, \frac{\boldsymbol{B}}{W^{2}}+\boldsymbol{v}(\boldsymbol{v} \cdot \boldsymbol{B})\right),
\end{aligned}
$$

where $W=1 / \sqrt{1-|\boldsymbol{v}|^{2}}$ is the Lorentz factor. It is easy to verify the following relations

$$
u^{\alpha} u_{\alpha}=-1, \quad u^{\alpha} b_{\alpha}=0, \quad|b|^{2} \equiv b^{\alpha} b_{\alpha}=\frac{|\boldsymbol{B}|^{2}}{W^{2}}+(\boldsymbol{v} \cdot \boldsymbol{B})^{2} .
$$

To close the system (1.1)-(1.4), this paper considers the equation of state (EOS) for the perfect gas

$$
p=(\Gamma-1) \rho e
$$


with the adiabatic index $\Gamma \in(1,2]$. The RHD case can be obtained by setting $\boldsymbol{B} \equiv \mathbf{0}$.

Numerical simulation is a powerful way to help us better understand the physical mechanisms in the RHDs and RMHDs. For the computational purpose, the system (1.1)-(1.7) is rewritten in a lab frame as follows

$$
\frac{\partial \boldsymbol{U}}{\partial t}+\sum_{k=1}^{d} \frac{\partial \boldsymbol{F}_{k}(\boldsymbol{U})}{\partial x_{k}}=0,
$$

with the divergence-free constraint on the magnetic field

$$
\sum_{k=1}^{d} \frac{\partial B_{k}}{\partial x_{k}}=0
$$

where $\boldsymbol{U}$ and $\boldsymbol{F}_{k}$ are respectively the conservative variable vector and the flux vector in the $x_{k^{-}}$ direction, and defined by

$$
\begin{aligned}
& \boldsymbol{U}=(D, \boldsymbol{m}, E, \boldsymbol{B})^{\mathrm{T}} \\
& \boldsymbol{F}_{k}=\left(D v_{k}, \boldsymbol{m} v_{k}-B_{k}\left(\boldsymbol{B} / W^{2}+(\boldsymbol{v} \cdot \boldsymbol{B}) \boldsymbol{v}\right)+p_{\mathrm{tot}} \boldsymbol{e}_{k}, m_{k}, v_{k} \boldsymbol{B}-B_{k} \boldsymbol{v}\right)^{\mathrm{T}}
\end{aligned}
$$

with the mass density $D=\rho W$, the momentum density $\boldsymbol{m}=\left(\rho h W^{2}+|\boldsymbol{B}|^{2}\right) \boldsymbol{v}-(\boldsymbol{v} \cdot \boldsymbol{B}) \boldsymbol{B}$, and the energy density $E=D h W-p_{\text {tot }}+|\boldsymbol{B}|^{2}$. Here $\boldsymbol{e}_{k}$ denotes the $k$-th row of the $d \times d$ unit matrix, and $p_{\text {tot }}$ denotes the total pressure containing the gas pressure $p$ and the magnetic pressure $p_{m}=\frac{1}{2}|b|^{2}$. Due to no explicit expression for the primitive variables $(\rho, \boldsymbol{v}, p, \boldsymbol{B})^{\mathrm{T}}$ and the flux $\boldsymbol{F}_{k}$ in terms of $\boldsymbol{U}$, a nonlinear algebraic equation, see e.g. [37], has to be solved in order to recover the values of the primitive variables and the flux from the given $\boldsymbol{U}$. It is obvious that the nonlinearity of (1.8)-(1.10) becomes much stronger than the non-relativistic case due to the relativistic effect, thus its analytical treatment is very challenging. The first numerical work may date back to the artificial viscosity method for the RHD equations in the Lagrangian coordinates [44, 45] and the Eulerian coordinates [61]. Since the early 1990s, the modern shock-capturing methods were extended to the RHD and RMHD equations, such as the Roe-type scheme [2, 22], the Harten-Lax-van Leer (HLL) method $[15,16,55]$, the Harten-Lax-van Leer-Contact (HLLC) method [39, 46, 47], the Harten-Laxvan Leer-Discontinuities (HLLD) method [50], the essentially non-oscillatory (ENO) and weighted ENO (WENO) methods [17, 15, 16], the piecewise parabolic methods [41, 49], the Runge-Kutta discontinuous Galerkin (DG) methods with WENO limiter [75, 76], the direct Eulerian generalized Riemann problem schemes $[71,72,70,66]$, the gas kinetics schemes $[12,13]$, the two-stage fourthorder time discretization [73], the adaptive moving mesh methods [31, 32], and so on. Recently, the properties of the admissible state set and the physical-constraints-preserving (both the rest-mass density and the kinetic pressure of the numerical solutions are positive and the magnitude of the fluid velocity is less than the speed of light) numerical schemes were well studied for the RHD and 
RMHD equations, see [39, 40, 65, 67, 68, 69, 63]. The readers are also referred to the early review articles $[25,42,43]$ for more references.

For the RHD and RMHD equations, the entropy condition is an important property which should be respected according to the second law of thermodynamics. On the other hand, it is well known that the weak solution of the quasi-linear hyperbolic conservation laws nay not be unique so that the entropy condition is needed to single out the unique physical relevant solution among all the weak solutions. Thus it is of great significance to seek the entropy stable (ES) schemes (satisfying some discrete or semi-discrete entropy conditions) for the quasi-linear system of hyperbolic conservation laws. For the scalar conservation laws, the fully-discrete conservative monotone schemes were nonlinearly stable and satisfied the entropy conditions, thus they could converge to the entropy solution $[30,14]$. A class of the so-called E-schemes satisfying the semidiscrete entropy conditions for any convex entropy was studied in [51, 52], but they were restricted to the first-order accuracy. Generally, it is difficult to show that the high-order schemes of the scalar conservation laws and the schemes for the system of hyperbolic conservation laws satisfy the entropy inequality for any convex entropy function. In [6], a second-order accurate scheme is shown to satisfy all the entropy conditions, which evolves not only the cell averages but also the solution values at half nodes. Many researchers are trying to study the high-order accurate ES schemes, which satisfy the entropy inequality for a given entropy pair. The two-point entropy conservative (EC) flux and corresponding second-order EC schemes (satisfying the semi-discrete entropy identity) were proposed in [56, 57], and their higher-order extension was studied in [38]. It is known that the EC schemes may become oscillatory near the shock waves so that some additional dissipation terms have to be added to obtain the ES schemes. Combining the EC flux with the "sign" property of the ENO reconstruction, the arbitrary high-order ES schemes were constructed by using high-order dissipation terms [24]. The ES schemes were then extended to the finite difference schemes based on summation-by-parts (SBP) operators [23]. Some ES schemes were also studied in the DG framework, such as the space-time DG formulation [34, 33], the DG spectral element methods [26, 8], and the nodal DG schemes on the simplex meshes [10]. More ES DG methods can be found in the review articles [11,27].

Recently, the high-order accurate ES finite difference schemes for the RHD equations were firstly studied in [19], in which the dissipation terms built on the fifth-order WENO reconstruction and the switch function in [5] was of the fifth-order accuracy and the "sign" property simultaneously. Later, the TeCNO scheme [24] was extended to the RHD equations [4], where the dissipation terms were based on the ENO reconstruction. For the ideal RMHDs, the high-order accurate ES finite difference schemes were proposed in [64] and the ES DG schemes were studied in [20] by using the 
symmetrizable RMHD equations and the suitable discretization of the source terms.

In view of the fact that the solutions of the RHD equations often exhibit localized structures, e.g. containing sharp transitions or discontinuities in relatively localized regions, the second-order accurate ES adaptive moving mesh schemes for the RHD equations are proposed in [21] to improve the efficiency and quality of numerical simulation. This paper is devoted to extend such ES adaptive moving mesh schemes as the high-order (greater than second-order) accurate schemes for the RHD and RMHD equations. The key point is the derivation of the higher-order accurate EC and ES finite difference schemes in the curvilinear coordinates. For such purpose, one should carefully deal with the discretization of the temporal and spatial metrics and the Jacobian introduced by the coordinate transformation and construct the high-order EC and ES fluxes with the discrete metrics. We prove that the suitable linear combinations of the two-point EC flux in the curvilinear coordinates give the high-order EC fluxes, which can be regarded as a refinement of the arbitrarily high-order accurate EC fluxes in the Cartesian coordinates in [38]. The spatial derivatives in the source terms of the symmetrizable RMHD equations and the geometric conservation laws are discretized by using the linear combinations of the corresponding second-order case to obtain high-order accuracy. Based on the proposed high-order accurate EC schemes and the high-order accurate dissipation terms built on the WENO reconstruction, the high-order accurate ES schemes are obtained for the RHD and RMHD equations in the curvilinear coordinates. Several two- and three-dimensional numerical tests are conducted to validate the shock-capturing ability and high efficiency of our high-order accurate ES adaptive moving mesh methods on the parallel computer system with the MPI communication. The numerical results show that the high-order accurate ES adaptive moving mesh schemes outperform both their counterparts on the uniform mesh and the second-order ES adaptive moving mesh schemes [21].

The paper is organized as follows. Section 2 gives the symmetrizable RMHD equations in the curvilinear coordinates and corresponding entropy conditions. It involves the special case of the RHD equations, i.e. (1.8)-(1.10) with $\boldsymbol{B} \equiv \mathbf{0}$. Section 3 presents the high-order accurate EC finite difference schemes in the curvilinear coordinates, while Section 4 gives the high-order accurate ES finite difference schemes by adding suitable dissipation terms based on the WENO reconstruction. The adaptive moving mesh strategy is introduced in Section 5. Several numerical tests are conducted in Section 6 to validate the high-order accuracy, the shock-capturing ability and the efficiency of the proposed schemes. Section 7 concludes the work with further remarks. 


\section{Entropy conditions for symmetrizable RMHD equations}

This section introduces some basic notations and the entropy conditions for the symmetrizable RMHD equations.

Definition 2.1. A strictly convex scalar function $\eta(\boldsymbol{U})$ is called an entropy function of the system (1.8) if there exists associated entropy fluxes $q_{k}(\boldsymbol{U})$ such that

$$
q_{k}^{\prime}(\boldsymbol{U})=\boldsymbol{V}^{\mathrm{T}} \boldsymbol{F}_{k}^{\prime}(\boldsymbol{U}), \quad k=1,2, \cdots, d,
$$

where $\boldsymbol{V}=\eta^{\prime}(\boldsymbol{U})^{\mathrm{T}}$ is called the entropy variables, and $\left(\eta, q_{k}\right)$ is an entropy pair.

For the smooth solutions of (1.8)-(1.10), multiplying (1.8) by $\boldsymbol{V}^{\mathrm{T}}$ gives the entropy identity

$$
\frac{\partial \eta(\boldsymbol{U})}{\partial t}+\sum_{k=1}^{d} \frac{\partial q_{k}(\boldsymbol{U})}{\partial x_{k}}=0
$$

However, if the solutions contain discontinuities, then the above identity does not hold and the weak solutions should be considered.

Definition 2.2. A weak solution $\boldsymbol{U}$ of (1.8)-(1.10) is called an entropy solution if for all entropy functions $\eta$, the inequality

$$
\frac{\partial \eta(\boldsymbol{U})}{\partial t}+\sum_{k=1}^{d} \frac{\partial q_{k}(\boldsymbol{U})}{\partial x_{k}} \leqslant 0
$$

holds in the sense of distributions.

For the system (1.8)-(1.10) with zero magnetic field $(\boldsymbol{B} \equiv \mathbf{0})$, the entropy pair can be defined by the thermodynamic entropy $[19,54]$ as follows

$$
\eta(\boldsymbol{U})=-\frac{\rho W s}{\Gamma-1}, \quad q_{k}(\boldsymbol{U})=\eta v_{k},
$$

where $s=\ln \left(p / \rho^{\Gamma}\right)$ is the thermodynamic entropy, $\eta$ is a convex function of $\boldsymbol{U}$ and $\left(\eta, q_{k}\right)$ satisfies the consistent condition (2.1). However, when $\boldsymbol{B} \not \equiv \mathbf{0}$, the function pair in (2.3) does not satisfy (2.1), and it can be verified that in general the system (1.8)-(1.10) cannot be symmetrized [20, 64]. Motivated by the symmetrization of the non-relativistic magnetohydrodynamics [28, 53], some source terms can be added to get a symmetrizable RMHD system as follows [64]

$$
\frac{\partial \boldsymbol{U}}{\partial t}+\sum_{k=1}^{d} \frac{\partial \boldsymbol{F}_{k}}{\partial x_{k}}=-\Phi^{\prime}(\boldsymbol{V})^{\mathrm{T}} \sum_{k=1}^{d} \frac{\partial B_{k}}{\partial x_{k}}, \boldsymbol{V}:=\eta^{\prime}(\boldsymbol{U})^{\mathrm{T}},
$$

where $\Phi(\boldsymbol{V})$ is a homogeneous function of degree one, i.e. $\Phi=\Phi^{\prime}(\boldsymbol{V}) \boldsymbol{V}$, with

$$
\Phi=\frac{\rho W(\boldsymbol{v} \cdot \boldsymbol{B})}{p}, \quad \Phi^{\prime}(\boldsymbol{V})=\left(0, \boldsymbol{B} / W^{2}+\boldsymbol{v}(\boldsymbol{v} \cdot \boldsymbol{B}), \boldsymbol{v} \cdot \boldsymbol{B}, \boldsymbol{v}\right)
$$


that is to say, the function pair in (2.3) can symmetrize the modified RMHD system (2.4) so that it is an entropy pair of (2.4). The entropy variable $\boldsymbol{V}$ can be explicit expressed as

$$
\boldsymbol{V}=\eta^{\prime}(\boldsymbol{U})^{\mathrm{T}}=\left(\frac{\Gamma-s}{\Gamma-1}+\frac{\rho}{p}, \frac{\rho W \boldsymbol{v}^{\mathrm{T}}}{p},-\frac{\rho W}{p}, \frac{\rho\left(\boldsymbol{B}+W^{2} \boldsymbol{v}(\boldsymbol{v} \cdot \boldsymbol{B})\right)}{p W}\right)^{\mathrm{T}} .
$$

For the smooth solutions, taking the dot product of $\boldsymbol{V}$ with (2.4) yields the entropy identity

$$
\frac{\partial \eta(\boldsymbol{U})}{\partial t}+\sum_{k=1}^{d} \frac{\partial q_{k}(\boldsymbol{U})}{\partial x_{k}}=0
$$

while for the discontinuous solutions, it is replaced with the entropy inequality

$$
\frac{\partial \eta(\boldsymbol{U})}{\partial t}+\sum_{k=1}^{d} \frac{\partial q_{k}(\boldsymbol{U})}{\partial x_{k}} \leqslant 0
$$

which holds in the sense of distributions. One can further define the entropy potential $\phi$ and entropy flux potential $\psi_{k}$ from the given $\left(\eta(\boldsymbol{U}), q_{k}(\boldsymbol{U})\right)$ and $\Phi(\boldsymbol{V})$ by

$$
\begin{aligned}
\phi & :=\boldsymbol{V}^{\mathrm{T}} \boldsymbol{U}-\eta(\boldsymbol{U})=\rho W+\frac{\rho W|b|^{2}}{2 p} \\
\psi_{k} & :=\boldsymbol{V}^{\mathrm{T}} \boldsymbol{F}_{k}(\boldsymbol{U})+\Phi(\boldsymbol{V}) B_{k}-q_{k}(\boldsymbol{U})=\rho v_{k} W+\frac{\rho v_{k} W|b|^{2}}{2 p},
\end{aligned}
$$

which are important in obtaining the sufficient condition for the two-point EC flux.

Similar to [21], let us derive the curvilinear coordinate form of the symmetrizable RMHD equations (2.4) and corresponding entropy conditions. Let $\Omega_{p}$ be the physical domain with coordinates $\boldsymbol{x}=\left(x_{1}, \cdots, x_{d}\right)$, in which (2.4) is specified, and $\Omega_{c}$ be the computational domain with coordinates $\boldsymbol{\xi}=\left(\xi_{1}, \cdots, \xi_{d}\right)$ that is artificially chosen for the sake of the mesh redistribution or movement. Our adaptive moving meshes for $\Omega_{p}$ can be generated as the images of a reference mesh in $\Omega_{c}$ by a time dependent, differentiable, one-to-one coordinate mapping $\boldsymbol{x}=\boldsymbol{x}(\tau, \boldsymbol{\xi})$, which can be written as

$$
t=\tau, \quad \boldsymbol{x}=\boldsymbol{x}(\tau, \boldsymbol{\xi}), \quad \boldsymbol{\xi}=\left(\xi_{1}, \cdots, \xi_{d}\right) \in \Omega_{c} .
$$

Under this transformation, the system $(2.4)$ in the coordinates $(\tau, \boldsymbol{\xi})$ reads

$$
\frac{\partial \mathcal{U}}{\partial \tau}+\sum_{k=1}^{d} \frac{\partial \mathcal{F}_{k}}{\partial \xi_{k}}=-\Phi^{\prime}(\boldsymbol{V})^{\mathrm{T}} \sum_{k=1}^{d} \frac{\partial \mathcal{B}_{k}}{\partial \xi_{k}}
$$

with

$$
\mathcal{U}=J \boldsymbol{U}, \mathcal{F}_{k}=\left(J \frac{\partial \xi_{k}}{\partial t} \boldsymbol{U}\right)+\sum_{j=1}^{d}\left(J \frac{\partial \xi_{k}}{\partial x_{j}} \boldsymbol{F}_{j}\right), \mathcal{B}_{k}=\sum_{j=1}^{d}\left(J \frac{\partial \xi_{k}}{\partial x_{j}} B_{j}\right)
$$


where $J$ denotes the determinant of the Jacobian matrix and its 3D version is explicitly given by

$$
J=\operatorname{det}\left(\frac{\partial(t, \boldsymbol{x})}{\partial(\tau, \boldsymbol{\xi})}\right)=\left|\begin{array}{cccc}
1 & 0 & 0 & 0 \\
\frac{\partial x_{1}}{\partial \tau} & \frac{\partial x_{1}}{\partial \xi_{1}} & \frac{\partial x_{1}}{\partial \xi_{2}} & \frac{\partial x_{1}}{\partial \xi_{3}} \\
\frac{\partial x_{2}}{\partial \tau} & \frac{\partial x_{2}}{\partial \xi_{1}} & \frac{\partial x_{2}}{\partial \xi_{2}} & \frac{\partial x_{2}}{\partial \xi_{3}} \\
\frac{\partial x_{3}}{\partial \tau} & \frac{\partial x_{3}}{\partial \xi_{1}} & \frac{\partial x_{3}}{\partial \xi_{2}} & \frac{\partial x_{3}}{\partial \xi_{3}}
\end{array}\right|
$$

The metrics should satisfy the following geometric conservation laws (GCLs) consisting of the volume conservation law (VCL) and the surface conservation laws (SCLs)

$$
\begin{array}{ll}
\text { VCL: } & \frac{\partial J}{\partial \tau}+\sum_{k=1}^{d} \frac{\partial}{\partial \xi_{k}}\left(J \frac{\partial \xi_{k}}{\partial t}\right)=0 \\
\text { SCLs: } & \sum_{k=1}^{d} \frac{\partial}{\partial \xi_{k}}\left(J \frac{\partial \xi_{k}}{\partial x_{j}}\right)=0, j=1, \cdots, d .
\end{array}
$$

The former indicates that the volumetric increment of a moving cell must be equal to the sum of the changes along the surfaces that enclose the volume, while the latter indicates that the cell volumes must be closed by its surfaces [74]. Those GCLs imply that free-stream solution is preserved by (2.8), in other words, a physical constant state is an exact solution of (2.8). Finally, by using the GCLs (2.9), see [21], the entropy identity for $(2.8)$ in the coordinates $(\tau, \boldsymbol{\xi})$ is

$$
\frac{\partial(J \eta)}{\partial \tau}+\sum_{k=1}^{d} \frac{\partial \mathfrak{q}_{k}}{\partial \xi_{k}}=0
$$

with

$$
\mathfrak{q}_{k}=\left(J \frac{\partial \xi_{k}}{\partial t} \eta\right)+\sum_{j=1}^{d}\left(J \frac{\partial \xi_{k}}{\partial x_{j}} q_{j}\right),
$$

but when the solutions are not smooth, it is replaced with the entropy inequality

$$
\frac{\partial(J \eta)}{\partial \tau}+\sum_{k=1}^{d} \frac{\partial \mathfrak{q}_{k}}{\partial \xi_{k}} \leqslant 0,
$$

which holds in the sense of distribution.

Remark 2.1. The continuous GCLs (2.9) are crucial in deriving the governing equations (2.8), the entropy identity (2.10) and the entropy inequality (2.11). Corresponding discrete GCLs will be important in proving the EC property of our schemes, see Section 3.

Remark 2.2. For the RHD case, the entropy variable $\boldsymbol{V}$, the entropy potential $\phi$, and the entropy flux potential $\psi_{k}$ can be obtained by setting $\boldsymbol{B} \equiv \mathbf{0}$. 


\section{High-order accurate EC schemes}

This section presents the 3D high-order accurate EC finite difference schemes for the RMHD system (2.8), which gives corresponding schemes for the RHD equations by setting $\boldsymbol{B} \equiv \mathbf{0}$. The $1 \mathrm{D}$ and 2D schemes in the curvilinear coordinates are given in Appendix A and Appendix B, respectively. For simplicity, only $3 \mathrm{D}$ adaptive moving mesh schemes for the RMHD equations in curvilinear coordinates (2.8) on structured meshes are presented hereafter.

\subsection{Two-point EC flux}

To develop the high-order accurate EC schemes, one of the main ingredient is the so-called two-point EC flux.

Definition 3.1. For the RMHD system (2.8), a numerical flux $\widetilde{\mathcal{F}}_{k}\left(\boldsymbol{U}_{l}, \boldsymbol{U}_{r},\left(J \frac{\partial \xi_{k}}{\partial \zeta}\right)_{l},\left(J \frac{\partial \xi_{k}}{\partial \zeta}\right)_{r}\right)$ is called two-point EC flux, $\zeta=t, x_{1}, x_{2}, x_{3}$, if it is consistent with $\mathcal{F}_{k}$ and satisfies

$$
\begin{aligned}
\left(\boldsymbol{V}\left(\boldsymbol{U}_{r}\right)-\boldsymbol{V}\left(\boldsymbol{U}_{l}\right)\right)^{\mathrm{T}} \widetilde{\mathcal{F}}_{k}= & \frac{1}{2}\left(\left(J \frac{\partial \xi_{k}}{\partial t}\right)_{l}+\left(J \frac{\partial \xi_{k}}{\partial t}\right)_{r}\right)\left(\phi\left(\boldsymbol{U}_{r}\right)-\phi\left(\boldsymbol{U}_{l}\right)\right) \\
& +\sum_{j=1}^{3} \frac{1}{2}\left(\left(J \frac{\partial \xi_{k}}{\partial x_{j}}\right)_{l}+\left(J \frac{\partial \xi_{k}}{\partial x_{j}}\right)_{r}\right)\left(\psi_{j}\left(\boldsymbol{U}_{r}\right)-\psi_{j}\left(\boldsymbol{U}_{l}\right)\right) \\
& -\sum_{j=1}^{3} \frac{1}{4}\left(\left(J \frac{\partial \xi_{k}}{\partial x_{j}}\right)_{l}+\left(J \frac{\partial \xi_{k}}{\partial x_{j}}\right)_{r}\right)\left(B_{j}\left(\boldsymbol{U}_{l}\right)+B_{j}\left(\boldsymbol{U}_{r}\right)\right)\left(\Phi\left(\boldsymbol{U}_{r}\right)-\Phi\left(\boldsymbol{U}_{l}\right)\right)
\end{aligned}
$$

where $\Phi$ and $\phi, \psi_{j}$ are defined in (2.5) and (2.6), respectively.

Remark 3.1. If $\boldsymbol{B} \equiv \mathbf{0}$, then (3.1) reduces to the RHD case [21], while, if $(t, \boldsymbol{x}) \equiv(\tau, \boldsymbol{\xi})$, then (3.1) reduces to the Cartesian coordinate case [64].

What follows is to find such a two-point EC flux satisfying (3.1). Similar to [21], the EC flux can be chosen as follows

$$
\widetilde{\mathcal{F}}_{k}\left(\boldsymbol{U}_{l}, \boldsymbol{U}_{r},\left(J \frac{\partial \xi_{k}}{\partial \zeta}\right)_{l},\left(J \frac{\partial \xi_{k}}{\partial \zeta}\right)_{r}\right)=\stackrel{\circ}{\boldsymbol{U}}(l ; r)+\sum_{j=1}^{3} \stackrel{\circ}{\boldsymbol{F}}_{j}(l ; r)
$$

where $\zeta=t, x_{1}, x_{2}, x_{3}$, and

$$
\stackrel{\circ}{\boldsymbol{U}}(l ; r)=\frac{1}{2}\left(\left(J \frac{\partial \xi_{k}}{\partial t}\right)_{l}+\left(J \frac{\partial \xi_{k}}{\partial t}\right)_{r}\right) \widetilde{\boldsymbol{U}}, \stackrel{\circ}{\boldsymbol{F}}_{j}(l ; r)=\frac{1}{2}\left(\left(J \frac{\partial \xi_{k}}{\partial x_{j}}\right)_{l}+\left(J \frac{\partial \xi_{k}}{\partial x_{j}}\right)_{r}\right) \widetilde{\boldsymbol{F}}_{j},
$$

with $\widetilde{\boldsymbol{U}}$ and $\widetilde{\boldsymbol{F}}_{j}$ satisfying

$$
\begin{aligned}
& \left(\boldsymbol{V}_{r}-\boldsymbol{V}_{l}\right)^{\mathrm{T}} \widetilde{\boldsymbol{U}}=\phi_{r}-\phi_{l}, \\
& \left(\boldsymbol{V}_{r}-\boldsymbol{V}_{l}\right)^{\mathrm{T}} \widetilde{\boldsymbol{F}}_{j}=\left[\left(\psi_{j}\right)_{r}-\left(\psi_{j}\right)_{l}\right]-\frac{1}{2}\left[\left(B_{j}\right)_{l}+\left(B_{j}\right)_{r}\right]\left(\Phi_{r}-\Phi_{l}\right) .
\end{aligned}
$$


For the RMHD system (2.8), $\widetilde{\boldsymbol{F}}_{j}^{\mathrm{RMHD}}$ in [20] is used, while $\widetilde{\boldsymbol{U}}^{\mathrm{RMHD}}$ can be given by following the derivation of $\widetilde{\boldsymbol{F}}_{j}^{\mathrm{RMHD}}$ and reads

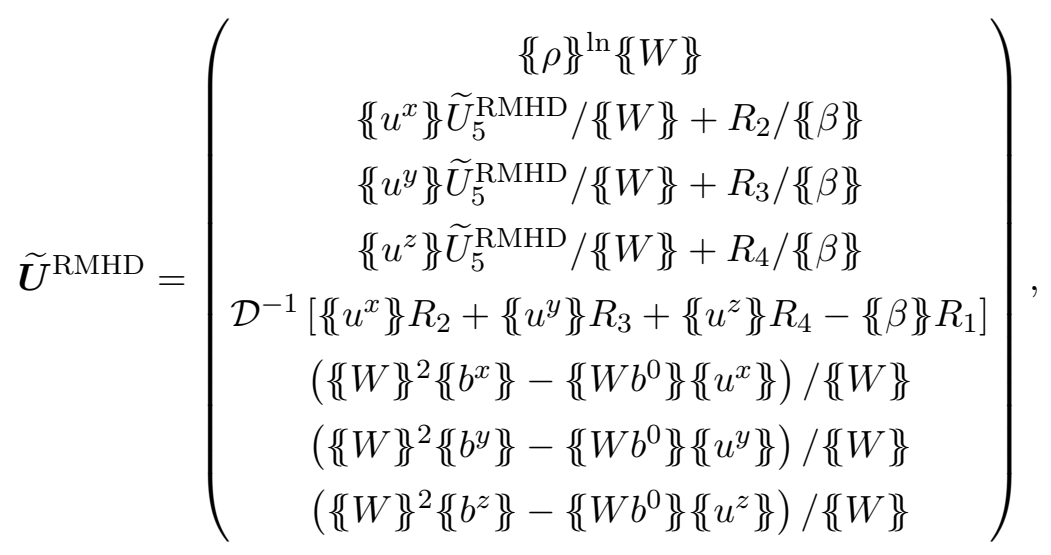

where

$$
\begin{aligned}
& \mathcal{D}=\left\{\{ \beta \} \left(\left\{\{W\}^{2}-\sum_{k=1}^{3}\left\{\left\{u^{k}\right\}^{2}\right) /\{\{W\}, \beta=\rho / p,\right.\right.\right. \\
& R_{1}=-\alpha_{0} \widetilde{U}_{1}^{\mathrm{RMHD}}-\frac{1}{2}\left\{\left\{W\left(b^{0}\right)^{2}\right\}\right\}+\sum_{k=1}^{2}\left[\frac{1}{2}\{\{W\}\}\left\{\left(b^{k}\right)^{2}\right\}-\left\{\left\{b^{k}\right\} \widetilde{U}_{k+5}^{\mathrm{RMHD}}\right],\right. \\
& R_{2}=\left(\alpha_{1}\left\{\left\{u^{x}\right\}\right\}-\left\{\{\beta\}\left\{\left\{W b^{0}\right\}\right\}\left\{\left\{b^{x}\right\}\right) /\{[W\},\right.\right. \\
& R_{3}=\left(\alpha _ { 1 } \left\{\left\{u^{y}\right\}-\left\{\{\beta\}\left\{\left\{W b^{0}\right\}\right\}\left\{\left\{b^{y}\right\}\right) /\{[W\},\right.\right.\right. \\
& R_{4}=\left(\alpha _ { 1 } \left\{\left\{u^{z}\right\}-\left\{\{\beta\}\left\{\left\{W b^{0}\right\}\left\{\left\{b^{z}\right\}\right\}\right) /\{\{W\},\right.\right.\right. \\
& \left.\alpha_{0}=1+1 /(\Gamma-1) /\{\beta\}\right\}^{\ln }, \\
& \alpha_{1}=\left\{\{\rho\}+\frac{1}{2}\{\{\beta\}\} \sum_{k=1}^{3}\left\{\left(b^{k}\right)^{2}\right\}\right\}+\frac{1}{2}\{\{\beta\}\}\left\{W\left(b^{0}\right)^{2}\right\} /\{\{W\},
\end{aligned}
$$

here $\left\{\{a\}^{\ln }=\llbracket a \rrbracket / \llbracket \ln a \rrbracket\right.$ is the logarithmic mean, see [35], and $\widetilde{\boldsymbol{U}}_{n}^{\mathrm{RMHD}}$ denotes the $n$th component of $\widetilde{\boldsymbol{U}}^{\text {RMHD }}$. For the RHD equations, a two-point EC flux in curvilinear coordinates can be found in $[21]$.

\subsection{Discretization of RMHD system and VCL}

Assume that the 3D computational domain $\Omega_{c}$ is chosen as a cuboid for convenience, e.g. $\left[a_{1}, b_{1}\right] \times\left[a_{2}, b_{2}\right] \times\left[a_{3}, b_{3}\right]$, and divided into a fixed orthogonal mesh $\left\{\left(\xi_{1, i_{1}}, \xi_{2, i_{2}}, \xi_{3, i_{3}}\right):\right.$

$\left.a_{k}=\xi_{k, 0}<\xi_{k, 1}<\cdots<\xi_{k, i_{k}}<\cdots<\xi_{k, N_{k}-1}=b_{k}, k=1,2,3\right\}$ with the constant step-size $\Delta \xi_{k}=\xi_{k, i_{k}+1}-\xi_{k, i_{k}}$. For the sake of brevity, the index $\boldsymbol{i}=\left(i_{1}, i_{2}, i_{3}\right)$ is used to denote the point $\left(\xi_{1, i_{1}}, \xi_{2, i_{2}}, \xi_{3, i_{3}}\right)$, and the subscript $\{\boldsymbol{i}, k, n\}$ denotes the index $\boldsymbol{i}$ increases $n$ in the $i_{k}$-direction, e.g., $\left\{\boldsymbol{i}, 3, \frac{1}{2}\right\}$ denotes $\left(i_{1}, i_{2}, i_{3}+\frac{1}{2}\right)$. 
Based on the above notations, consider the following $2 p$ th-order $(p \geq 1)$ semi-discrete conservative finite difference schemes for the RMHD system (2.8) and the VCL (2.9)

$$
\begin{aligned}
\frac{\mathrm{d}}{\mathrm{d} t} \mathcal{U}_{\boldsymbol{i}} & =-\sum_{k=1}^{3} \frac{1}{\Delta \xi_{k}}\left(\left(\widetilde{\mathcal{F}_{k}}\right)_{\boldsymbol{i}, k,+\frac{1}{2}}^{2 p \text { th }}-\left(\widetilde{\mathcal{F}_{k}}\right)_{\boldsymbol{i}, k,-\frac{1}{2}}^{2 p \mathrm{th}}\right)-\Phi^{\prime}\left(\boldsymbol{V}_{\boldsymbol{i}}\right)^{\mathrm{T}} \sum_{k=1}^{3} \frac{1}{\Delta \xi_{k}}\left(\left(\widetilde{\mathcal{B}_{k}}\right)_{\boldsymbol{i}, k,+\frac{1}{2}}^{2 p \text { th }}-\left(\widetilde{\mathcal{B}_{k}}\right)_{\boldsymbol{i}, k,-\frac{1}{2}}^{2 p \text { th }}\right), \\
\frac{\mathrm{d}}{\mathrm{d} t} J_{\boldsymbol{i}} & \left.=-\sum_{k=1}^{3} \frac{1}{\Delta \xi_{k}}\left(\widetilde{J \frac{\partial \xi_{k}}{\partial t}}\right)_{\boldsymbol{i}, k,+\frac{1}{2}}^{2 p \mathrm{th}}-\left(\widetilde{J \frac{\partial \xi_{k}}{\partial t}}\right)_{i, k,-\frac{1}{2}}^{2 p \text { th }}\right),
\end{aligned}
$$

where $J_{\boldsymbol{i}}(t)$ and $\mathcal{U}_{\boldsymbol{i}}(t)$ approximate the point values of $J(t, \boldsymbol{\xi})$ and $\mathcal{U}(t, \boldsymbol{\xi})$ at $\boldsymbol{i}$, respectively, and $\left(\widetilde{\mathcal{F}_{k}}\right)_{\boldsymbol{i}, k, \pm \frac{1}{2}}^{2 p \text { th }},\left(\widetilde{\mathcal{B}_{k}}\right)_{i, k, \pm \frac{1}{2}}^{2 p \text { th }},\left(\widetilde{J \frac{\partial \xi_{k}}{\partial t}}\right)_{i, k, \pm \frac{1}{2}}^{2 p \text { th }}$ are the numerical fluxes used in the approximations of the flux derivative, source terms, and spatial derivatives in the VCL, respectively.

The high-order $(p>1)$ accurate EC schemes (3.4) for the system (2.8) are mainly built on the following parts.

1. For the given entropy pair, the two-point EC flux $\widetilde{\mathcal{F}_{k}}$ is first derived from (3.1), and then the high-order EC flux $\left(\widetilde{\mathcal{F}}_{k}\right)_{i, k, \pm \frac{1}{2}}^{2 p \text { th }}$ is gotten by some linear combination of the two-point EC flux $\widetilde{\mathcal{F}}_{k}$ in (3.2), such that the approximation of the flux derivative $\frac{\partial \mathcal{F}_{k}}{\partial \xi_{k}}$ is $2 p$ th-order accurate. It is considered as an extension of the high-order accurate EC schemes in the Cartesian coordinates [38] to the curvilinear coordinates.

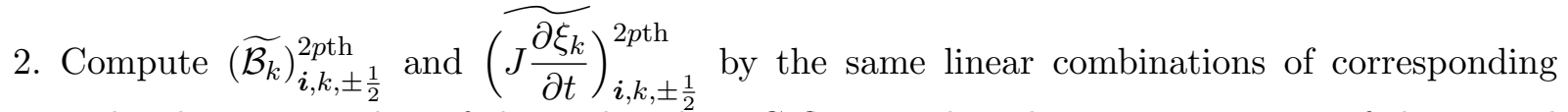
2nd-order case as that of the $2 p$ th-order EC flux, so that the approximations of the spatial derivatives in source terms and the VCL are also $2 p$ th-order accurate. The discretization of the latter degenerates to the $2 p$ th-order accurate central difference.

3. The metrics $\left(\widetilde{J \frac{\partial \xi_{k}}{\partial x_{j}}}\right)_{i}$ used in the above two parts are discretized by the $2 p$ th-order central difference based on the conservative metrics method (CMM) [60] such that the SCLs hold in the discrete level.

4. The schemes (3.4)-(3.5) can be proved to be $2 p$ th-order accurate and EC by combing the above three parts, which mimics the derivation of the continuous entropy identity (2.10) in the curvilinear coordinates.

The first two parts are given in Proposition 3.1, the third is addressed in Section 3.3, and the last one is summarized in Theorem 3.2.

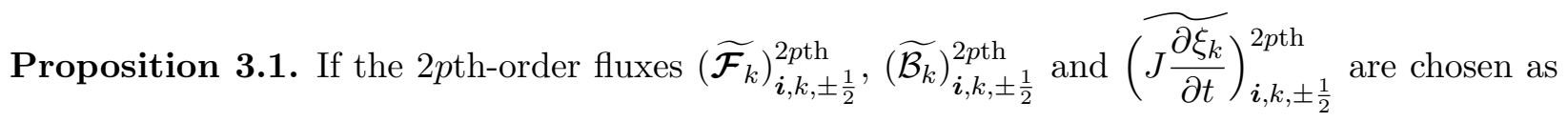


follows

$$
\begin{aligned}
& \left(\widetilde{\mathcal{F}_{k}}\right)_{i, k,+\frac{1}{2}}^{2 p \text { th }}=\sum_{n=1}^{p} \alpha_{p, n} \sum_{s=0}^{n-1} \widetilde{\mathcal{F}_{k}}\left(\boldsymbol{U}_{\boldsymbol{i}, k,-s}, \boldsymbol{U}_{\boldsymbol{i}, k,-s+n},\left(J \frac{\partial \xi_{k}}{\partial \zeta}\right)_{\boldsymbol{i}, k,-s},\left(J \frac{\partial \xi_{k}}{\partial \zeta}\right)_{\boldsymbol{i}, k,-s+n}\right), \\
& \left(\widetilde{\mathcal{B}_{k}}\right)_{\boldsymbol{i}, k,+\frac{1}{2}}^{2 p \text { th }}=\sum_{n=1}^{p} \alpha_{p, n} \sum_{s=0}^{n-1} \widetilde{\mathcal{B}_{k}}\left(B_{\boldsymbol{i}, k,-s}, B_{\boldsymbol{i}, k,-s+n},\left(J \frac{\partial \xi_{k}}{\partial x_{j}}\right)_{\boldsymbol{i}, k,-s},\left(J \frac{\partial \xi_{k}}{\partial x_{j}}\right)_{\boldsymbol{i}, k,-s+n}\right), \\
& \left(\widetilde{J \frac{\partial \xi_{k}}{\partial \zeta}}\right)_{\boldsymbol{i}, k,+\frac{1}{2}}^{2 p \text { th }}=\sum_{n=1}^{p} \alpha_{p, n} \sum_{s=0}^{n-1}\left(\widetilde{\partial \frac{\partial \xi_{k}}{\partial \zeta}}\right)\left(\left(J \frac{\partial \xi_{k}}{\partial \zeta}\right)_{\boldsymbol{i}, k,-s},\left(J \frac{\partial \xi_{k}}{\partial \zeta}\right)_{\boldsymbol{i}, k,-s+n}\right),
\end{aligned}
$$

where $\zeta=t, x_{1}, x_{2}, x_{3}, \widetilde{\mathcal{B}_{k}}$ and $\left(\widetilde{J \frac{\partial \xi_{k}}{\partial \zeta}}\right)$ are corresponding 2 nd-order case as follows

$$
\begin{aligned}
& \widetilde{\mathcal{B}_{k}}\left(\left(B_{j}\right)_{l},\left(B_{j}\right)_{r},\left(J \frac{\partial \xi_{k}}{\partial x_{j}}\right)_{l},\left(J \frac{\partial \xi_{k}}{\partial x_{j}}\right)_{r}\right)=\sum_{j=1}^{3} \frac{1}{4}\left(\left(J \frac{\partial \xi_{k}}{\partial x_{j}}\right)_{l}+\left(J \frac{\partial \xi_{k}}{\partial x_{j}}\right)_{r}\right)\left(\left(B_{j}\right)_{l}+\left(B_{j}\right)_{r}\right), \\
& \widetilde{\left(J \frac{\partial \xi_{k}}{\partial \zeta}\right)}\left(\left(J \frac{\partial \xi_{k}}{\partial \zeta}\right)_{l},\left(J \frac{\partial \xi_{k}}{\partial \zeta}\right)_{r}\right)=\frac{1}{2}\left(\left(J \frac{\partial \xi_{k}}{\partial \zeta}\right)_{l}+\left(J \frac{\partial \xi_{k}}{\partial \zeta}\right)_{r}\right)
\end{aligned}
$$

and the coefficients in the linear combinations satisfy the constraints [38]

$$
\sum_{n=1}^{p} n \alpha_{p, n}=1, \sum_{n=1}^{p} n^{2 s-1} \alpha_{p, n}=0, s=2, \cdots, p,
$$

then the approximation of the flux derivative $\frac{\partial \mathcal{F}_{k}}{\partial \xi_{k}}$ is $2 p$ th-order accurate, i.e.

$$
\frac{1}{\Delta \xi_{k}}\left(\left(\widetilde{\mathcal{F}}_{k}\right)_{i, k,+\frac{1}{2}}^{2 p \text { th }}-\left(\widetilde{\mathcal{F}}_{k}\right)_{i, k,-\frac{1}{2}}^{2 p \text { th }}\right)=\left.\frac{\partial \mathcal{F}_{k}}{\partial \xi_{k}}\right|_{\boldsymbol{i}}+\mathcal{O}\left(\Delta \xi_{k}^{2 p}\right), k=1,2,3
$$

Similarly, the approximations of the source terms and the spatial derivatives in the VCL are also $2 p$ th-order accurate.

To prove such proposition, let us first consider the following Lemma.

Lemma 3.1. If the smooth two-parameter scalar function $\widetilde{f}\left(u\left(\zeta_{l}\right), u\left(\zeta_{r}\right)\right)$ and vector-value function $\widetilde{\boldsymbol{F}}\left(\boldsymbol{U}\left(\zeta_{l}\right), \boldsymbol{U}\left(\zeta_{r}\right)\right)$ satisfy

$$
\begin{array}{cl}
\text { Consistency } & \widetilde{f}(u, u)=f(u), \widetilde{\boldsymbol{F}}(\boldsymbol{U}, \boldsymbol{U})=\boldsymbol{F}(\boldsymbol{U}), \\
\text { Symmetry } & \widetilde{f}\left(u\left(\zeta_{l}\right), u\left(\zeta_{r}\right)\right)=\widetilde{f}\left(u\left(\zeta_{r}\right), u\left(\zeta_{l}\right)\right), \widetilde{\boldsymbol{F}}\left(\boldsymbol{U}\left(\zeta_{l}\right), \boldsymbol{U}\left(\zeta_{r}\right)\right)=\widetilde{\boldsymbol{F}}\left(\boldsymbol{U}\left(\zeta_{r}\right), \boldsymbol{U}\left(\zeta_{l}\right)\right),
\end{array}
$$

then the following identities hold

$$
\left.2 \frac{\partial}{\partial \zeta_{r}} \widetilde{f}\left(u\left(\zeta_{l}\right), u\left(\zeta_{r}\right)\right)\right|_{\zeta_{r}=\zeta_{l}}=\left.\frac{\partial}{\partial \zeta} f(u(\zeta))\right|_{\zeta=\zeta_{l}},
$$




$$
\begin{aligned}
& \left.2 \frac{\partial}{\partial \zeta_{r}} \widetilde{\boldsymbol{F}}\left(\boldsymbol{U}\left(\zeta_{l}\right), \boldsymbol{U}\left(\zeta_{r}\right)\right)\right|_{\zeta_{r}=\zeta_{l}}=\left.\frac{\partial}{\partial \zeta} \boldsymbol{F}(\boldsymbol{U}(\zeta))\right|_{\zeta=\zeta_{l}}, \\
& \left.2 \frac{\partial}{\partial \zeta_{r}}\left[\widetilde{f}\left(u\left(\zeta_{l}\right), u\left(\zeta_{r}\right)\right) \widetilde{\boldsymbol{F}}\left(\boldsymbol{U}\left(\zeta_{l}\right), \boldsymbol{U}\left(\zeta_{r}\right)\right)\right]\right|_{\zeta_{r}=\zeta_{l}}=\left.\frac{\partial}{\partial \zeta}[f(u(\zeta)) \boldsymbol{F}(\boldsymbol{U}(\zeta))]\right|_{\zeta=\zeta_{l}} .
\end{aligned}
$$

Proof. The first identity is a special case of the second, which comes from [9]. Utilizing the symmetry and the consistency of $\widetilde{\boldsymbol{F}}$ gives

$$
\begin{aligned}
\left.2 \frac{\partial \widetilde{\boldsymbol{F}}\left(\boldsymbol{U}_{l}, \boldsymbol{U}_{r}\right)}{\partial \boldsymbol{U}_{r}}\right|_{\boldsymbol{U}_{r}=\boldsymbol{U}_{l}} & =\left.\left(\frac{\partial \widetilde{\boldsymbol{F}}\left(\boldsymbol{U}_{l}, \boldsymbol{U}_{r}\right)}{\partial \boldsymbol{U}_{l}}+\frac{\partial \widetilde{\boldsymbol{F}}\left(\boldsymbol{U}_{l}, \boldsymbol{U}_{r}\right)}{\partial \boldsymbol{U}_{r}}\right)\right|_{\boldsymbol{U}_{r}=\boldsymbol{U}_{l}} \\
& =\left.\frac{\partial \widetilde{\boldsymbol{F}}(\boldsymbol{U}, \boldsymbol{U})}{\partial \boldsymbol{U}}\right|_{\boldsymbol{U}=\boldsymbol{U}_{l}}=\left.\frac{\partial \boldsymbol{F}(\boldsymbol{U})}{\partial \boldsymbol{U}}\right|_{\boldsymbol{U}=\boldsymbol{U}_{l}}
\end{aligned}
$$

Letting $\boldsymbol{U}_{l}=\boldsymbol{U}\left(\zeta_{l}\right), \boldsymbol{U}_{r}=\boldsymbol{U}\left(\zeta_{r}\right)$ and using the chain rule gives

$$
\left.2 \frac{\partial}{\partial \zeta_{r}} \widetilde{\boldsymbol{F}}\left(\boldsymbol{U}\left(\zeta_{l}\right), \boldsymbol{U}\left(\zeta_{r}\right)\right)\right|_{\zeta_{r}=\zeta_{l}}=\left.\frac{\partial}{\partial \zeta} \boldsymbol{F}(\boldsymbol{U}(\zeta))\right|_{\zeta=\zeta_{l}} .
$$

The third identity can be obtained as follows

$$
\begin{aligned}
& \left.2 \frac{\partial}{\partial \zeta_{r}}\left[\widetilde{f}\left(u\left(\zeta_{l}\right), u\left(\zeta_{r}\right)\right) \widetilde{\boldsymbol{F}}\left(\boldsymbol{U}\left(\zeta_{l}\right), \boldsymbol{U}\left(\zeta_{r}\right)\right)\right]\right|_{\zeta_{r}=\zeta_{l}} \\
= & \left.2 \frac{\partial}{\partial \zeta_{r}} \widetilde{f}\left(u\left(\zeta_{l}\right), u\left(\zeta_{r}\right)\right)\right|_{\zeta_{r}=\zeta_{l}} \boldsymbol{F}\left(\boldsymbol{U}\left(\zeta_{l}\right)\right)+\left.2 f\left(u\left(\zeta_{l}\right)\right) \frac{\partial}{\partial \zeta_{r}} \widetilde{\boldsymbol{F}}\left(\boldsymbol{U}\left(\zeta_{l}\right), \boldsymbol{U}\left(\zeta_{r}\right)\right)\right|_{\zeta_{r}=\zeta_{l}} \\
= & \left.\frac{\partial}{\partial \zeta} f(u(\zeta))\right|_{\zeta=\zeta_{l}} \boldsymbol{F}\left(\boldsymbol{U}\left(\zeta_{l}\right)\right)+\left.f\left(u\left(\zeta_{l}\right)\right) \frac{\partial}{\partial \zeta} \boldsymbol{F}(\boldsymbol{U}(\zeta))\right|_{\zeta=\zeta_{l}} \\
= & \left.\frac{\partial}{\partial \zeta}[f(u(\zeta)) \boldsymbol{F}(\boldsymbol{U}(\zeta))]\right|_{\zeta=\zeta_{l}},
\end{aligned}
$$

where the first equality uses the product rule.

Based on the above Lemma, it is ready to prove Proposition 3.1.

Proof. It suffices to consider the $i_{k}$-direction and to assume the other two independent variables to be fixed and omitted in the following expressions by using “...". If taking $\zeta_{l}=\hat{\xi}_{k}, \zeta_{r}=\tilde{\xi}_{k}$, and

$$
\begin{gathered}
\tilde{f}=\frac{1}{2}\left(\left(J \frac{\partial \xi_{k}}{\partial x_{j}}\right)\left(\cdots, \hat{\xi}_{k}, \cdots\right)+\left(J \frac{\partial \xi_{k}}{\partial x_{j}}\right)\left(\cdots, \tilde{\xi}_{k}, \cdots\right)\right), \\
\widetilde{\boldsymbol{F}}=\widetilde{\boldsymbol{F}}_{j}\left(\boldsymbol{U}\left(\cdots, \hat{\xi}_{k}, \cdots\right), \boldsymbol{U}\left(\cdots, \tilde{\xi}_{k}, \cdots\right)\right),
\end{gathered}
$$

in Proposition 3.1, then one has

$$
\begin{aligned}
& \left.\frac{\partial}{\partial \tilde{\xi}_{k}}\left[\frac{1}{2}\left(\left(J \frac{\partial \xi_{k}}{\partial x_{j}}\right)\left(\cdots, \hat{\xi}_{k}, \cdots\right)+\left(J \frac{\partial \xi_{k}}{\partial x_{j}}\right)\left(\cdots, \tilde{\xi}_{k}, \cdots\right)\right) \widetilde{\boldsymbol{F}}_{j}\left(\boldsymbol{U}\left(\cdots, \hat{\xi}_{k}, \cdots\right), \boldsymbol{U}\left(\cdots, \tilde{\xi}_{k}, \cdots\right)\right)\right]\right|_{\tilde{\xi}_{k}=\hat{\xi}_{k}} \\
= & \left.\frac{1}{2} \frac{\partial}{\partial \xi_{k}}\left[\left(J \frac{\partial \xi_{k}}{\partial x_{j}}\right)\left(\cdots, \xi_{k}, \cdots\right) \boldsymbol{F}_{j}\left(\boldsymbol{U}\left(\ldots, \xi_{k}, \ldots\right)\right)\right]\right|_{\xi_{k}=\hat{\xi}_{k}} .
\end{aligned}
$$


If utilizing (3.14), then one can expand $\stackrel{\circ}{\boldsymbol{F}}_{j}(\boldsymbol{i} ; \boldsymbol{i}, k, \pm n)$ defined in (3.3) at $\xi_{k, i_{k}}$ by using Taylor series as follows

$$
\begin{aligned}
\stackrel{\circ}{\boldsymbol{F}}_{j}(\boldsymbol{i} ; \boldsymbol{i}, k, \pm n)= & {\left[\left(J \frac{\partial \xi_{k}}{\partial x_{j}}\right) \boldsymbol{F}_{j}\right]_{i} \pm \frac{n \Delta \xi_{k}}{2} \frac{\partial}{\partial \xi_{k}}\left[\left(J \frac{\partial \xi_{k}}{\partial x_{j}}\right) \boldsymbol{F}_{j}\right]_{\boldsymbol{i}} } \\
& +\sum_{s=2}^{2 p} \frac{\left( \pm n \Delta \xi_{k}\right)^{s}}{s !} \partial_{\xi_{k}}^{s} \stackrel{\circ}{\boldsymbol{F}}_{j}(\boldsymbol{i} ; \boldsymbol{i})+\mathcal{O}\left(\Delta \xi_{k}^{2 p+1}\right)
\end{aligned}
$$

so that their difference becomes

$$
\begin{aligned}
\stackrel{\circ}{\boldsymbol{F}}_{j}(\boldsymbol{i} ; \boldsymbol{i}, k,+n)-\stackrel{\circ}{\boldsymbol{F}}_{j}(\boldsymbol{i} ; \boldsymbol{i}, k,-n)= & n \Delta \xi_{k} \frac{\partial}{\partial \xi_{k}}\left[\left(J \frac{\partial \xi_{k}}{\partial x_{j}}\right) \boldsymbol{F}_{j}\right]_{\boldsymbol{i}} \\
& +2 \sum_{s=2}^{p} \frac{\left(n \Delta \xi_{k}\right)^{2 s-1}}{(2 s-1) !} \partial_{\xi_{k}}^{2 s-1} \stackrel{\circ}{\boldsymbol{F}}_{j}(\boldsymbol{i} ; \boldsymbol{i})+\mathcal{O}\left(\Delta \xi_{k}^{2 p+1}\right) .
\end{aligned}
$$

Similarly, it can be verified that

$$
\begin{aligned}
\stackrel{\circ}{\boldsymbol{U}}(\boldsymbol{i} ; \boldsymbol{i}, k,+n)-\stackrel{\circ}{\boldsymbol{U}}(\boldsymbol{i} ; \boldsymbol{i}, k,-n)= & n \Delta \xi_{k} \frac{\partial}{\partial \xi_{k}}\left[\left(J \frac{\partial \xi_{k}}{\partial t}\right) \boldsymbol{U}\right]_{\boldsymbol{i}} \\
& +2 \sum_{s=2}^{p} \frac{\left(n \Delta \xi_{k}\right)^{2 s-1}}{(2 s-1) !} \partial_{\xi_{k}}^{2 s-1} \stackrel{\boldsymbol{U}}{ }(\boldsymbol{i} ; \boldsymbol{i})+\mathcal{O}\left(\Delta \xi_{k}^{2 p+1}\right) .
\end{aligned}
$$

Based on those, one gets

$$
\begin{aligned}
& \frac{1}{\Delta \xi_{k}}\left(\left(\widetilde{\mathcal{F}}_{k}\right)_{i, k,+\frac{1}{2}}^{2 p \text { th }}-\left(\widetilde{\mathcal{F}}_{k}\right)_{i, k,-\frac{1}{2}}^{2 p \text { th }}\right) \\
& =\sum_{n=1}^{p} \alpha_{p, n}\left(\stackrel{\circ}{\boldsymbol{U}}(\boldsymbol{i} ; \boldsymbol{i}, k,+n)+\sum_{j=1}^{3} \stackrel{\circ}{\boldsymbol{F}}_{j}(\boldsymbol{i} ; \boldsymbol{i}, k,+n)-\stackrel{\circ}{\boldsymbol{U}}(\boldsymbol{i} ; \boldsymbol{i}, k,-n)-\sum_{j=1}^{3} \stackrel{\circ}{\boldsymbol{F}}_{j}(\boldsymbol{i} ; \boldsymbol{i}, k,-n)\right) \\
& =\left.\left(\sum_{n=1}^{p} n \alpha_{p, n}\right) \frac{\partial \mathcal{F}_{k}}{\partial \xi_{k}}\right|_{i}+\frac{2 \Delta \xi_{k}^{2 s-2}}{(2 s-1) !} \sum_{s=2}^{p}\left(\sum_{n=1}^{p} n^{2 s-1} \alpha_{p, n}\right) \frac{\partial^{2 s-1}\left(\stackrel{\circ}{\boldsymbol{U}}(\boldsymbol{i} ; \boldsymbol{i})+\sum_{j=1}^{3} \stackrel{\circ}{\boldsymbol{F}}_{j}(\boldsymbol{i} ; \boldsymbol{i})\right)}{\partial \xi_{k}^{2 s-1}}+\mathcal{O}\left(\Delta \xi_{k}^{2 p}\right) \\
& =\left.\frac{\partial \mathcal{F}_{k}}{\partial \xi_{k}}\right|_{i}+\mathcal{O}\left(\Delta \xi_{k}^{2 p}\right), k=1,2,3
\end{aligned}
$$

where the last equality uses the constraints (3.11). Similarly it can be proved that the approximations of the source terms and the spatial derivatives in the VCL are also $2 p$ th-order accurate.

\subsection{Discrete GCLs}

This section introduces some appropriate discretizations of the spatial metrics $\left(J \frac{\partial \xi_{k}}{\partial x_{j}}\right)_{i}$ and the temporal metrics $\left(J \frac{\partial \xi_{k}}{\partial t}\right)_{i}$ in order to get the discrete SCLs

$$
\sum_{k=1}^{3} \frac{1}{\Delta \xi_{k}}\left(\left(\widetilde{J \frac{\partial \xi_{k}}{\partial x_{j}}}\right)_{i, k,+\frac{1}{2}}^{2 p \text { th }}-\left(\widetilde{J \frac{\partial \xi_{k}}{\partial x_{j}}}\right)_{i, k,-\frac{1}{2}}^{2 p \text { th }}\right)=0, j=1,2,3 .
$$


and the discrete VCL.

For the smooth transformation (2.7), the following identities hold

$$
\begin{aligned}
& J \frac{\partial \xi_{1}}{\partial x_{1}}=\frac{\partial x_{2}}{\partial \xi_{2}} \frac{\partial x_{3}}{\partial \xi_{3}}-\frac{\partial x_{2}}{\partial \xi_{3}} \frac{\partial x_{3}}{\partial \xi_{2}}, J \frac{\partial \xi_{1}}{\partial x_{2}}=\frac{\partial x_{3}}{\partial \xi_{2}} \frac{\partial x_{1}}{\partial \xi_{3}}-\frac{\partial x_{3}}{\partial \xi_{3}} \frac{\partial x_{1}}{\partial \xi_{2}}, J \frac{\partial \xi_{1}}{\partial x_{3}}=\frac{\partial x_{1}}{\partial \xi_{2}} \frac{\partial x_{2}}{\partial \xi_{3}}-\frac{\partial x_{1}}{\partial \xi_{3}} \frac{\partial x_{2}}{\partial \xi_{2}} \\
& J \frac{\partial \xi_{2}}{\partial x_{1}}=\frac{\partial x_{2}}{\partial \xi_{3}} \frac{\partial x_{3}}{\partial \xi_{1}}-\frac{\partial x_{2}}{\partial \xi_{1}} \frac{\partial x_{3}}{\partial \xi_{3}}, J \frac{\partial \xi_{2}}{\partial x_{2}}=\frac{\partial x_{3}}{\partial \xi_{3}} \frac{\partial x_{1}}{\partial \xi_{1}}-\frac{\partial x_{3}}{\partial \xi_{1}} \frac{\partial x_{1}}{\partial \xi_{3}}, J \frac{\partial \xi_{2}}{\partial x_{3}}=\frac{\partial x_{1}}{\partial \xi_{3}} \frac{\partial x_{2}}{\partial \xi_{1}}-\frac{\partial x_{1}}{\partial \xi_{1}} \frac{\partial x_{2}}{\partial \xi_{3}} \\
& J \frac{\partial \xi_{3}}{\partial x_{1}}=\frac{\partial x_{2}}{\partial \xi_{1}} \frac{\partial x_{3}}{\partial \xi_{2}}-\frac{\partial x_{2}}{\partial \xi_{2}} \frac{\partial x_{3}}{\partial \xi_{1}}, J \frac{\partial \xi_{3}}{\partial x_{2}}=\frac{\partial x_{3}}{\partial \xi_{1}} \frac{\partial x_{1}}{\partial \xi_{2}}-\frac{\partial x_{3}}{\partial \xi_{2}} \frac{\partial x_{1}}{\partial \xi_{1}}, J \frac{\partial \xi_{3}}{\partial x_{3}}=\frac{\partial x_{1}}{\partial \xi_{1}} \frac{\partial x_{2}}{\partial \xi_{2}}-\frac{\partial x_{1}}{\partial \xi_{2}} \frac{\partial x_{2}}{\partial \xi_{1}}
\end{aligned}
$$

which can be reformulated into the divergence form

$$
\begin{aligned}
J \frac{\partial \xi_{1}}{\partial x_{1}} & =\frac{\partial}{\partial \xi_{3}}\left(\frac{\partial x_{2}}{\partial \xi_{2}} x_{3}\right)-\frac{\partial}{\partial \xi_{2}}\left(\frac{\partial x_{2}}{\partial \xi_{3}} x_{3}\right), J \frac{\partial \xi_{1}}{\partial x_{2}}=\frac{\partial}{\partial \xi_{3}}\left(\frac{\partial x_{3}}{\partial \xi_{2}} x_{1}\right)-\frac{\partial}{\partial \xi_{2}}\left(\frac{\partial x_{3}}{\partial \xi_{3}} x_{1}\right), \\
J \frac{\partial \xi_{1}}{\partial x_{3}} & =\frac{\partial}{\partial \xi_{3}}\left(\frac{\partial x_{1}}{\partial \xi_{2}} x_{2}\right)-\frac{\partial}{\partial \xi_{2}}\left(\frac{\partial x_{1}}{\partial \xi_{3}} x_{2}\right), \\
J \frac{\partial \xi_{2}}{\partial x_{1}} & =\frac{\partial}{\partial \xi_{1}}\left(\frac{\partial x_{2}}{\partial \xi_{3}} x_{3}\right)-\frac{\partial}{\partial \xi_{3}}\left(\frac{\partial x_{2}}{\partial \xi_{1}} x_{3}\right), J \frac{\partial \xi_{2}}{\partial x_{2}}=\frac{\partial}{\partial \xi_{1}}\left(\frac{\partial x_{3}}{\partial \xi_{3}} x_{1}\right)-\frac{\partial}{\partial \xi_{3}}\left(\frac{\partial x_{3}}{\partial \xi_{1}} x_{1}\right), \\
J \frac{\partial \xi_{2}}{\partial x_{3}} & =\frac{\partial}{\partial \xi_{1}}\left(\frac{\partial x_{1}}{\partial \xi_{3}} x_{2}\right)-\frac{\partial}{\partial \xi_{3}}\left(\frac{\partial x_{1}}{\partial \xi_{1}} x_{2}\right), \\
J \frac{\partial \xi_{3}}{\partial x_{1}} & =\frac{\partial}{\partial \xi_{2}}\left(\frac{\partial x_{2}}{\partial \xi_{1}} x_{3}\right)-\frac{\partial}{\partial \xi_{1}}\left(\frac{\partial x_{2}}{\partial \xi_{2}} x_{3}\right), J \frac{\partial \xi_{3}}{\partial x_{2}}=\frac{\partial}{\partial \xi_{2}}\left(\frac{\partial x_{3}}{\partial \xi_{1}} x_{1}\right)-\frac{\partial}{\partial \xi_{1}}\left(\frac{\partial x_{3}}{\partial \xi_{2}} x_{1}\right), \\
J \frac{\partial \xi_{3}}{\partial x_{3}} & =\frac{\partial}{\partial \xi_{2}}\left(\frac{\partial x_{1}}{\partial \xi_{1}} x_{2}\right)-\frac{\partial}{\partial \xi_{1}}\left(\frac{\partial x_{1}}{\partial \xi_{2}} x_{2}\right) .
\end{aligned}
$$

Those are useful to compute the discrete metrics and to obtain the discrete SCLs by the CMM [60]. Using the same discretizations for the first-order spatial derivatives in (3.16) gives

$$
\begin{aligned}
& \left(J \frac{\partial \xi_{1}}{\partial x_{1}}\right)_{i}=\frac{1}{\Delta \xi_{2} \Delta \xi_{3}}\left(\delta_{3}\left[\delta_{2}\left[x_{2}\right] x_{3}\right]-\delta_{2}\left[\delta_{3}\left[x_{2}\right] x_{3}\right]\right),\left(J \frac{\partial \xi_{1}}{\partial x_{2}}\right)_{i}=\frac{1}{\Delta \xi_{2} \Delta \xi_{3}}\left(\delta_{3}\left[\delta_{2}\left[x_{3}\right] x_{1}\right]-\delta_{2}\left[\delta_{3}\left[x_{3}\right] x_{1}\right]\right), \\
& \left(J \frac{\partial \xi_{1}}{\partial x_{3}}\right)_{i}=\frac{1}{\Delta \xi_{2} \Delta \xi_{3}}\left(\delta_{3}\left[\delta_{2}\left[x_{1}\right] x_{2}\right]-\delta_{2}\left[\delta_{3}\left[x_{1}\right] x_{2}\right]\right), \\
& \left(J \frac{\partial \xi_{2}}{\partial x_{1}}\right)_{i}=\frac{1}{\Delta \xi_{3} \Delta \xi_{1}}\left(\delta_{1}\left[\delta_{3}\left[x_{2}\right] x_{3}\right]-\delta_{3}\left[\delta_{1}\left[x_{2}\right] x_{3}\right]\right),\left(J \frac{\partial \xi_{2}}{\partial x_{2}}\right)_{i}=\frac{1}{\Delta \xi_{3} \Delta \xi_{1}}\left(\delta_{1}\left[\delta_{3}\left[x_{3}\right] x_{1}\right]-\delta_{3}\left[\delta_{1}\left[x_{3}\right] x_{1}\right]\right), \\
& \left(J \frac{\partial \xi_{2}}{\partial x_{3}}\right)_{i}=\frac{1}{\Delta \xi_{3} \Delta \xi_{1}}\left(\delta_{1}\left[\delta_{3}\left[x_{1}\right] x_{2}\right]-\delta_{3}\left[\delta_{1}\left[x_{1}\right] x_{2}\right]\right), \\
& \left(J \frac{\partial \xi_{3}}{\partial x_{1}}\right)_{i}=\frac{1}{\Delta \xi_{1} \Delta \xi_{2}}\left(\delta_{2}\left[\delta_{1}\left[x_{2}\right] x_{3}\right]-\delta_{1}\left[\delta_{2}\left[x_{2}\right] x_{3}\right]\right),\left(J \frac{\partial \xi_{3}}{\partial x_{2}}\right)_{i}=\frac{1}{\Delta \xi_{1} \Delta \xi_{2}}\left(\delta_{2}\left[\delta_{1}\left[x_{3}\right] x_{1}\right]-\delta_{1}\left[\delta_{2}\left[x_{3}\right] x_{1}\right]\right), \\
& \left(J \frac{\partial \xi_{3}}{\partial x_{3}}\right)_{i}=\frac{1}{\Delta \xi_{1} \Delta \xi_{2}}\left(\delta_{2}\left[\delta_{1}\left[x_{1}\right] x_{2}\right]-\delta_{1}\left[\delta_{2}\left[x_{1}\right] x_{2}\right]\right),
\end{aligned}
$$

where

$$
\delta_{k}\left[a_{\boldsymbol{i}}\right]=\frac{1}{2} \sum_{n=1}^{p} \alpha_{p, n}\left(a_{\boldsymbol{i}, k,+n}-a_{\boldsymbol{i}, k,-n}\right)
$$


is the $2 p$ th-order central difference operator in the $i_{k}$-direction. Combing the above discretizations with the fluxes (3.8), one can verify that the discrete SCLs (3.15) are satisfied. For example, for $j=1$, one has

$$
\begin{aligned}
& \sum_{k=1}^{3} \frac{1}{\Delta \xi_{k}}\left(\left(\widetilde{J \frac{\partial \xi_{k}}{\partial x_{1}}}\right)_{i, k,+\frac{1}{2}}^{2 p \text { th }}-\left(\widetilde{J \frac{\partial \xi_{k}}{\partial x_{1}}}\right)_{i, k,-\frac{1}{2}}^{2 p \text { th }}\right)=\sum_{k=1}^{3} \frac{1}{\Delta \xi_{k}} \delta_{k}\left[\left(J \frac{\partial \xi_{k}}{\partial x_{1}}\right)\right] \\
= & \frac{1}{\Delta \xi_{1}} \delta_{1}\left[\left(J \frac{\partial \xi_{1}}{\partial x_{1}}\right)\right]+\frac{1}{\Delta \xi_{2}} \delta_{2}\left[\left(J \frac{\partial \xi_{2}}{\partial x_{1}}\right)\right]+\frac{1}{\Delta \xi_{3}} \delta_{3}\left[\left(J \frac{\partial \xi_{3}}{\partial x_{1}}\right)\right] \\
= & \frac{1}{\Delta \xi_{1} \Delta \xi_{2} \Delta \xi_{3}}\left(\delta_{1} \delta_{3}\left[\delta_{2}\left[x_{2}\right] x_{3}\right]-\delta_{1} \delta_{2}\left[\delta_{3}\left[x_{2}\right] x_{3}\right]+\delta_{2} \delta_{1}\left[\delta_{3}\left[x_{2}\right] x_{3}\right]\right. \\
& \left.-\delta_{2} \delta_{3}\left[\delta_{1}\left[x_{2}\right] x_{3}\right]+\delta_{3} \delta_{2}\left[\delta_{1}\left[x_{2}\right] x_{3}\right]-\delta_{3} \delta_{1}\left[\delta_{2}\left[x_{2}\right] x_{3}\right]\right)=0,
\end{aligned}
$$

since $\delta_{j}$ and $\delta_{k}$ are commutative, i.e. $\delta_{j} \delta_{k}=\delta_{k} \delta_{j}$.

The temporal metrics $\left(J \partial_{t} \xi_{k}\right)$ satisfy

$$
J \frac{\partial \xi_{k}}{\partial t}=-\sum_{j=1}^{3} \frac{\partial x_{j}}{\partial t}\left(J \frac{\partial \xi_{k}}{\partial x_{j}}\right), k=1,2,3,
$$

so that one has the following approximation

$$
\left(J \frac{\partial \xi_{k}}{\partial t}\right)_{i}=-\sum_{j=1}^{3}\left(\dot{x}_{j}\right)_{i}\left(J \frac{\partial \xi_{k}}{\partial x_{j}}\right)_{i},
$$

where $\left(\dot{x}_{j}\right)_{i}, j=1,2,3$ are the grid velocities at $\boldsymbol{i}$, which will be provided by some given expressions or solving the mesh equations in Section 5. Since the quantities $\left(J \frac{\partial \xi_{k}}{\partial x_{j}}\right)_{i}$ have been obtained in (3.17), the implementation of (3.18) is simple and cheap. Combining (3.18) with (3.5) and (3.8) gives the semi-discrete VCL. Moreover, it can be verified the following free-stream preserving property.

Proposition 3.2. If the semi-discrete schemes (3.4)-(3.5) are integrated in time with the explicit SSP RK scheme from $t=t^{n}$ to $t^{n+1}=t^{n}+\Delta t^{n}$, with the time step size $\Delta t^{n}$, then the resulting fully-discrete schemes preserve the free-stream states.

Proof. The forward Euler time discretization is considered here, since the explicit SSP RK schemes are a convex combination of the forward Euler time discretizations. Assuming that $\boldsymbol{U}_{\boldsymbol{i}}^{n}=\boldsymbol{U}_{0}$ is a physical constant state, rewrite the update of the metric Jacobian $J_{\boldsymbol{i}}$ and the solution $\boldsymbol{U}_{\boldsymbol{i}}$ as follows

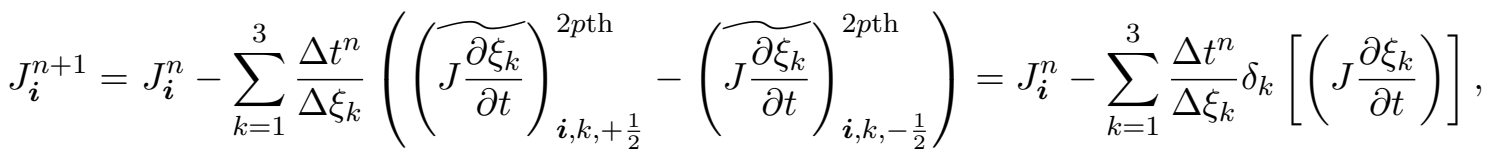

$$
\begin{aligned}
& (J \boldsymbol{U})_{\boldsymbol{i}}^{n+1}=(J \boldsymbol{U})_{i}^{n}-\sum_{k=1}^{3} \frac{\Delta t^{n}}{\Delta \xi_{k}}\left(\left(\widetilde{\mathcal{F}}_{k}\right)_{\boldsymbol{i}, k,+\frac{1}{2}}^{2 p t h}-\left(\widetilde{\mathcal{F}}_{k}\right)_{i, k,-\frac{1}{2}}^{2 p \text { th }}\right)
\end{aligned}
$$




$$
\begin{aligned}
= & J_{\boldsymbol{i}}^{n} \boldsymbol{U}_{0}-\sum_{k=1}^{3} \frac{\Delta t^{n}}{\Delta \xi_{k}} \sum_{n=1}^{p} \alpha_{p, n}[ \\
& +\frac{1}{2}\left(\left(J \frac{\partial \xi_{k}}{\partial t}\right)_{\boldsymbol{i}}+\left(J \frac{\partial \xi_{k}}{\partial t}\right)_{\boldsymbol{i}, k,+n}\right) \boldsymbol{U}_{0}+\sum_{j=1}^{3} \frac{1}{2}\left(\left(J \frac{\partial \xi_{k}}{\partial x_{j}}\right)_{\boldsymbol{i}}+\left(J \frac{\partial \xi_{k}}{\partial x_{j}}\right)_{\boldsymbol{i}, k,+n}\right) \boldsymbol{F}_{j}\left(\boldsymbol{U}_{0}\right) \\
& \left.-\frac{1}{2}\left(\left(J \frac{\partial \xi_{k}}{\partial t}\right)_{\boldsymbol{i}, k,-n}+\left(J \frac{\partial \xi_{k}}{\partial t}\right)_{i}\right) \boldsymbol{U}_{0}-\sum_{j=1}^{3} \frac{1}{2}\left(\left(J \frac{\partial \xi_{k}}{\partial x_{j}}\right)_{\boldsymbol{i}, k,-n}+\left(J \frac{\partial \xi_{k}}{\partial x_{j}}\right)_{\boldsymbol{i}}\right) \boldsymbol{F}_{j}\left(\boldsymbol{U}_{0}\right)\right] \\
= & \left(J_{\boldsymbol{i}}^{n}-\sum_{k=1}^{3} \frac{\Delta t^{n}}{\Delta \xi_{k}} \delta_{k}\left[\left(J \frac{\partial \xi_{k}}{\partial t}\right)\right]\right)_{\boldsymbol{U}_{0}}-\sum_{j=1}^{3}\left(\sum_{k=1}^{3} \frac{\Delta t^{n}}{\Delta \xi_{k}} \delta_{k}\left[\left(J \frac{\partial \xi_{k}}{\partial x_{j}}\right)\right]\right) \boldsymbol{F}_{j}\left(\boldsymbol{U}_{0}\right) \\
= & J_{\boldsymbol{i}}^{n+1} \boldsymbol{U}_{0},
\end{aligned}
$$

where the discrete GCLs have been used in the last equality. Thus $\boldsymbol{U}_{\boldsymbol{i}}^{n+1}=(J \boldsymbol{U})_{\boldsymbol{i}}^{n+1} / J_{\boldsymbol{i}}^{n+1}=\boldsymbol{U}_{0}$. The proof is completed.

\subsection{Proof of high-order accuracy and EC property}

This section is devoted to present the high-order accurate EC schemes based on the previous results.

Theorem 3.2. The semi-discrete schemes (3.4)-(3.5) with the fluxes (3.6)-(3.8) are $2 p$ th-order accurate and EC in the sense that

$$
\frac{\mathrm{d}}{\mathrm{d} t} J_{\boldsymbol{i}} \eta\left(\boldsymbol{U}_{\boldsymbol{i}}(t)\right)+\sum_{k=1}^{3} \frac{1}{\Delta \xi_{k}}\left(\left(\widetilde{\mathfrak{q}_{k}}\right)_{\boldsymbol{i}, k,+\frac{1}{2}}^{2 p \text { th }}-\left(\widetilde{\mathfrak{q}_{k}}\right)_{i, k,-\frac{1}{2}}^{2 p \text { th }}\right)=0
$$

with the consistent numerical entropy fluxes

$$
\left(\widetilde{\mathfrak{q}_{k}}\right)_{\boldsymbol{i}, k,+\frac{1}{2}}^{2 p \text { th }}=\sum_{n=1}^{p} \alpha_{p, n} \sum_{s=0}^{n-1} \widetilde{\mathfrak{q}_{k}}\left(\boldsymbol{U}_{\boldsymbol{i}, k,-s}, \boldsymbol{U}_{\boldsymbol{i}, k,-s+n},\left(J \frac{\partial \xi_{k}}{\partial \zeta}\right)_{\boldsymbol{i}, k,-s},\left(J \frac{\partial \xi_{k}}{\partial \zeta}\right)_{\boldsymbol{i}, k,-s+n}\right)
$$

where

$$
\begin{aligned}
& \widetilde{\mathfrak{q}_{k}}\left(\boldsymbol{U}_{l}, \boldsymbol{U}_{r},\left(J \frac{\partial \xi_{k}}{\partial \zeta}\right)_{l},\left(J \frac{\partial \xi_{k}}{\partial \zeta}\right)_{r}\right) \\
= & \frac{1}{2}\left(\boldsymbol{V}\left(\boldsymbol{U}_{l}\right)+\boldsymbol{V}\left(\boldsymbol{U}_{r}\right)\right)^{\mathrm{T}} \widetilde{\mathcal{F}_{k}}\left(\boldsymbol{U}_{l}, \boldsymbol{U}_{r},\left(J \frac{\partial \xi_{k}}{\partial \zeta}\right)_{l},\left(J \frac{\partial \xi_{k}}{\partial \zeta}\right)_{r}\right) \\
& -\frac{1}{4}\left(\left(J \frac{\partial \xi_{k}}{\partial t}\right)_{l}+\left(J \frac{\partial \xi_{k}}{\partial t}\right)_{r}\right)\left(\phi\left(\boldsymbol{U}_{l}\right)+\phi\left(\boldsymbol{U}_{r}\right)\right) \\
& -\sum_{j=1}^{3} \frac{1}{4}\left(\left(J \frac{\partial \xi_{k}}{\partial x_{j}}\right)_{l}+\left(J \frac{\partial \xi_{k}}{\partial x_{j}}\right)_{r}\right)\left(\psi_{j}\left(\boldsymbol{U}_{l}\right)+\psi_{j}\left(\boldsymbol{U}_{r}\right)\right) \\
& +\sum_{j=1}^{3} \frac{1}{8}\left(\left(J \frac{\partial \xi_{k}}{\partial x_{j}}\right)_{l}+\left(J \frac{\partial \xi_{k}}{\partial x_{j}}\right)_{r}\right)\left(\left(B_{j}\right)_{l}+\left(B_{j}\right)_{r}\right)\left(\Phi\left(\boldsymbol{U}_{l}\right)+\Phi\left(\boldsymbol{U}_{r}\right)\right) .
\end{aligned}
$$


Proof. From Proposition 3.1 and the discretizations of the metrics $\left(J \frac{\partial \xi_{k}}{\partial \zeta}\right)_{i}$ in (3.17) and (3.18), $\zeta=t, x_{1}, x_{2}, x_{3}$, it is obvious that the semi-discrete schemes (3.4)-(3.5) are $2 p$ th-order accurate in space.

Taking the dot product of (3.4) with $\boldsymbol{V}_{\boldsymbol{i}}$ and using the chain rule and the semi-discrete VCL (3.5) gives

$$
\begin{aligned}
\frac{\mathrm{d}}{\mathrm{d} t}\left(J_{\boldsymbol{i}} \eta_{\boldsymbol{i}}\right)= & -\sum_{k=1}^{3} \frac{1}{\Delta \xi_{k}}\left\{\boldsymbol{V}_{\boldsymbol{i}}^{\mathrm{T}}\left(\left(\widetilde{\mathcal{F}_{k}}\right)_{i, k,+\frac{1}{2}}^{2 p \text { th }}-\left(\widetilde{\mathcal{F}_{k}}\right)_{\boldsymbol{i}, k,-\frac{1}{2}}^{2 p \mathrm{th}}\right)-\phi_{\boldsymbol{i}}\left(\left(\widetilde{J \frac{\partial \xi_{k}}{\partial t}}\right)_{\boldsymbol{i}, k,+\frac{1}{2}}^{2 p \text { th }}-\left(\widetilde{J \frac{\partial \xi_{k}}{\partial t}}\right)_{i, k,-\frac{1}{2}}^{2 p \mathrm{th}}\right)\right. \\
& \left.+\Phi_{\boldsymbol{i}}\left(\left(\widetilde{\mathcal{B}_{k}}\right)_{\boldsymbol{i}, k,+\frac{1}{2}}^{2 p \mathrm{th}}-\left(\widetilde{\mathcal{B}_{k}}\right)_{\boldsymbol{i}, k,-\frac{1}{2}}^{2 p \mathrm{th}}\right)\right\} .
\end{aligned}
$$

Further utilizing the discrete SCLs (3.15) can get

$$
\begin{aligned}
\frac{\mathrm{d}}{\mathrm{d} t}\left(J_{\boldsymbol{i}} \eta_{\boldsymbol{i}}\right)= & -\sum_{k=1}^{3} \frac{1}{\Delta \xi_{k}}\left\{\boldsymbol{V}_{\boldsymbol{i}}^{\mathrm{T}}\left(\left(\widetilde{\mathcal{F}_{k}}\right)_{i, k,+\frac{1}{2}}^{2 p \text { th }}-\left(\widetilde{\mathcal{F}_{k}}\right)_{\boldsymbol{i}, k,-\frac{1}{2}}^{2 p \text { th }}\right)-\phi_{\boldsymbol{i}}\left(\left(\widetilde{J \frac{\partial \xi_{k}}{\partial t}}\right)_{\boldsymbol{i}, k,+\frac{1}{2}}^{2 p \text { th }}-\left(\widetilde{J \frac{\partial \xi_{k}}{\partial t}}\right)_{\boldsymbol{i}, k,-\frac{1}{2}}^{2 p \text { th }}\right)\right. \\
& \left.\left.\left.-\sum_{j=1}^{3}\left(\psi_{j}\right)_{\boldsymbol{i}}\left(\widetilde{\partial \frac{\partial \xi_{k}}{\partial x_{j}}}\right)_{\boldsymbol{i}, k,+\frac{1}{2}}^{2 p \text { th }}-\left(\widetilde{\partial \xi_{k}}\right)_{i, k,-\frac{1}{2}}^{2 p \text { th }}\right)+\Phi_{\boldsymbol{i}}\left(\widetilde{\left(\widetilde{\mathcal{B}}_{k}\right.}\right)_{\boldsymbol{i}, k,+\frac{1}{2}}^{2 p \text { th }}-\left(\widetilde{\mathcal{B}_{k}}\right)_{\boldsymbol{i}, k,-\frac{1}{2}}^{2 p \text { th }}\right)\right\} \\
= & -\sum_{k=1}^{3} \sum_{n=1}^{p} \frac{\alpha_{p, n}}{\Delta \xi_{k}}\left(I_{1}-I_{2}-I_{3}+I_{4}\right),
\end{aligned}
$$

where

$$
\begin{aligned}
I_{1}= & \boldsymbol{V}_{\boldsymbol{i}}^{\mathrm{T}}\left[\widetilde{\mathcal{F}}_{k}\left(\boldsymbol{U}_{\boldsymbol{i}}, \boldsymbol{U}_{\boldsymbol{i}, k,+n},\left(J \frac{\partial \xi_{k}}{\partial \zeta}\right)_{\boldsymbol{i}},\left(J \frac{\partial \xi_{k}}{\partial \zeta}\right)_{\boldsymbol{i}, k,+n}\right)-\widetilde{\mathcal{F}}_{k}\left(\boldsymbol{U}_{\boldsymbol{i}}, \boldsymbol{U}_{\boldsymbol{i}, k,-n},\left(J \frac{\partial \xi_{k}}{\partial \zeta}\right)_{\boldsymbol{i}},\left(J \frac{\partial \xi_{k}}{\partial \zeta}\right)_{\boldsymbol{i}, k,-n}\right)\right] \\
I_{2}= & \phi_{\boldsymbol{i}}\left[\frac{1}{2}\left(\left(J \frac{\partial \xi_{k}}{\partial t}\right)_{\boldsymbol{i}}+\left(J \frac{\partial \xi_{k}}{\partial t}\right)_{\boldsymbol{i}, k,+n}\right)\right] \\
I_{3}= & \left.\sum_{j=1}^{3}\left(\psi_{j}\right)_{\boldsymbol{i}}\left[\frac{1}{2}\left(\left(J \frac{\partial \xi_{k}}{\partial t}\right)_{\boldsymbol{i}}+\left(J \frac{\partial \xi_{k}}{\partial t}\right)_{\boldsymbol{i}, k,-n}\right)_{\boldsymbol{i}}+\left(J \frac{\partial \xi_{k}}{\partial x_{j}}\right)_{\boldsymbol{i}, k,+n}\right)-\frac{1}{2}\left(\left(J \frac{\partial \xi_{k}}{\partial x_{j}}\right)_{\boldsymbol{i}}+\left(J \frac{\partial \xi_{k}}{\partial x_{j}}\right)_{\boldsymbol{i}, k,-n}\right)\right] \\
I_{4}= & \sum_{j=1}^{3} \Phi_{\boldsymbol{i}}\left[\frac{1}{4}\left(\left(J \frac{\partial \xi_{k}}{\partial x_{j}}\right)_{\boldsymbol{i}}+\left(J \frac{\partial \xi_{k}}{\partial x_{j}}\right)_{\boldsymbol{i}, k,+n}\right)\left(\left(B_{j}\right)_{\boldsymbol{i}}+\left(B_{j}\right)_{\boldsymbol{i}, k,+n}\right)\right. \\
& \left.-\frac{1}{4}\left(\left(J \frac{\partial \xi_{k}}{\partial x_{j}}\right)_{\boldsymbol{i}}+\left(J \frac{\partial \xi_{k}}{\partial x_{j}}\right)_{\boldsymbol{i}, k,-n}\right)\left(\left(B_{j}\right)_{\boldsymbol{i}}+\left(B_{j}\right)_{\boldsymbol{i}, k,-n}\right)\right]
\end{aligned}
$$

If splitting $\boldsymbol{V}_{\boldsymbol{i}}$ as $\frac{1}{2}\left(\boldsymbol{V}_{\boldsymbol{i}}+\boldsymbol{V}_{\boldsymbol{i}, k,+n}\right)-\frac{1}{2}\left(\boldsymbol{V}_{\boldsymbol{i}, k,+n}-\boldsymbol{V}_{\boldsymbol{i}}\right)$ or $\frac{1}{2}\left(\boldsymbol{V}_{\boldsymbol{i}, k,-n}+\boldsymbol{V}_{\boldsymbol{i}}\right)+\frac{1}{2}\left(\boldsymbol{V}_{\boldsymbol{i}}-\boldsymbol{V}_{\boldsymbol{i}, k,-n}\right)$, then $I_{1}$ goes to

$$
I_{1}=+\frac{1}{2}\left(\boldsymbol{V}_{\boldsymbol{i}}+\boldsymbol{V}_{\boldsymbol{i}, k,+n}\right)^{\mathrm{T}} \widetilde{\mathcal{F}}_{k}\left(\boldsymbol{U}_{\boldsymbol{i}}, \boldsymbol{U}_{\boldsymbol{i}, k,+n},\left(J \frac{\partial \xi_{k}}{\partial \zeta}\right)_{\boldsymbol{i}},\left(J \frac{\partial \xi_{k}}{\partial \zeta}\right)_{\boldsymbol{i}, k,+n}\right)
$$




$$
\begin{aligned}
& -\frac{1}{2}\left(\boldsymbol{V}_{\boldsymbol{i}, k,+n}-\boldsymbol{V}_{\boldsymbol{i}}\right)^{\mathrm{T}} \widetilde{\mathcal{F}}_{k}\left(\boldsymbol{U}_{\boldsymbol{i}}, \boldsymbol{U}_{\boldsymbol{i}, k,+n},\left(J \frac{\partial \xi_{k}}{\partial \zeta}\right)_{\boldsymbol{i}},\left(J \frac{\partial \xi_{k}}{\partial \zeta}\right)_{\boldsymbol{i}, k,+n}\right) \\
& -\frac{1}{2}\left(\boldsymbol{V}_{\boldsymbol{i}}+\boldsymbol{V}_{\boldsymbol{i}, k,-n}\right)^{\mathrm{T}} \widetilde{\mathcal{F}}_{k}\left(\boldsymbol{U}_{\boldsymbol{i}}, \boldsymbol{U}_{\boldsymbol{i}, k,-n},\left(J \frac{\partial \xi_{k}}{\partial \zeta}\right)_{\boldsymbol{i}},\left(J \frac{\partial \xi_{k}}{\partial \zeta}\right)_{\boldsymbol{i}, k,-n}\right) \\
& -\frac{1}{2}\left(\boldsymbol{V}_{\boldsymbol{i}}-\boldsymbol{V}_{\boldsymbol{i}, k,-n}\right)^{\mathrm{T}} \widetilde{\mathcal{F}}_{k}\left(\boldsymbol{U}_{\boldsymbol{i}}, \boldsymbol{U}_{\boldsymbol{i}, k,-n},\left(J \frac{\partial \xi_{k}}{\partial \zeta}\right)_{\boldsymbol{i}},\left(J \frac{\partial \xi_{k}}{\partial \zeta}\right)_{\boldsymbol{i}, k,-n}\right) .
\end{aligned}
$$

Similarly, treating $\phi_{\boldsymbol{i}},\left(\psi_{j}\right)_{\boldsymbol{i}}$ and $\Phi_{\boldsymbol{i}}$ gives

$$
\begin{aligned}
& I_{2}=+\frac{1}{4}\left(\phi_{\boldsymbol{i}}+\phi_{\boldsymbol{i}, k,+n}\right)\left(\left(J \frac{\partial \xi_{k}}{\partial t}\right)_{\boldsymbol{i}}+\left(J \frac{\partial \xi_{k}}{\partial t}\right)_{\boldsymbol{i}, k,+n}\right)-\frac{1}{4}\left(\phi_{\boldsymbol{i}, k,+n}-\phi_{\boldsymbol{i}}\right)\left(\left(J \frac{\partial \xi_{k}}{\partial t}\right)_{\boldsymbol{i}}+\left(J \frac{\partial \xi_{k}}{\partial t}\right)_{\boldsymbol{i}, k,+n}\right) \\
& -\frac{1}{4}\left(\phi_{\boldsymbol{i}}+\phi_{\boldsymbol{i}, k,-n}\right)\left(\left(J \frac{\partial \xi_{k}}{\partial t}\right)_{\boldsymbol{i}}+\left(J \frac{\partial \xi_{k}}{\partial t}\right)_{\boldsymbol{i}, k,-n}\right)-\frac{1}{4}\left(\phi_{\boldsymbol{i}}-\phi_{\boldsymbol{i}, k,-n}\right)\left(\left(J \frac{\partial \xi_{k}}{\partial t}\right)_{\boldsymbol{i}}+\left(J \frac{\partial \xi_{k}}{\partial t}\right)_{\boldsymbol{i}, k,-n}\right) \text {, } \\
& I_{3}=+\sum_{j=1}^{3}\left[\frac{1}{4}\left(\left(\psi_{j}\right)_{i}+\left(\psi_{j}\right)_{i, k,+n}\right)\left(\left(J \frac{\partial \xi_{k}}{\partial x_{j}}\right)_{i}+\left(J \frac{\partial \xi_{k}}{\partial x_{j}}\right)_{i, k,+n}\right)\right. \\
& -\frac{1}{4}\left(\left(\psi_{j}\right)_{i, k,+n}-\left(\psi_{j}\right)_{i}\right)\left(\left(J \frac{\partial \xi_{k}}{\partial x_{j}}\right)_{i}+\left(J \frac{\partial \xi_{k}}{\partial x_{j}}\right)_{i, k,+n}\right) \\
& -\frac{1}{4}\left(\left(\psi_{j}\right)_{i}+\left(\psi_{j}\right)_{i, k,-n}\right)\left(\left(J \frac{\partial \xi_{k}}{\partial x_{j}}\right)_{i}+\left(J \frac{\partial \xi_{k}}{\partial x_{j}}\right)_{i, k,-n}\right) \\
& \left.-\frac{1}{4}\left(\left(\psi_{j}\right)_{i}-\left(\psi_{j}\right)_{i, k,-n}\right)\left(\left(J \frac{\partial \xi_{k}}{\partial x_{j}}\right)_{i}+\left(J \frac{\partial \xi_{k}}{\partial x_{j}}\right)_{i, k,-n}\right)\right] \\
& I_{4}=+\sum_{j=1}^{3}\left[\frac{1}{8}\left(\Phi_{\boldsymbol{i}}+\Phi_{\boldsymbol{i}, k,+n}\right)\left(\left(J \frac{\partial \xi_{k}}{\partial x_{j}}\right)_{\boldsymbol{i}}+\left(J \frac{\partial \xi_{k}}{\partial x_{j}}\right)_{\boldsymbol{i}, k,+n}\right)\left(\left(B_{j}\right)_{\boldsymbol{i}}+\left(B_{j}\right)_{\boldsymbol{i}, k,+n}\right)\right. \\
& -\frac{1}{8}\left(\Phi_{i, k,+n}-\Phi_{\boldsymbol{i}}\right)\left(\left(J \frac{\partial \xi_{k}}{\partial x_{j}}\right)_{i}+\left(J \frac{\partial \xi_{k}}{\partial x_{j}}\right)_{i, k,+n}\right)\left(\left(B_{j}\right)_{i}+\left(B_{j}\right)_{i, k,+n}\right) \\
& -\frac{1}{8}\left(\Phi_{\boldsymbol{i}}+\Phi_{\boldsymbol{i}, k,-n}\right)\left(\left(J \frac{\partial \xi_{k}}{\partial x_{j}}\right)_{\boldsymbol{i}}+\left(J \frac{\partial \xi_{k}}{\partial x_{j}}\right)_{\boldsymbol{i}, k,-n}\right)\left(\left(B_{j}\right)_{\boldsymbol{i}}+\left(B_{j}\right)_{\boldsymbol{i}, k,-n}\right) \\
& \left.-\frac{1}{8}\left(\Phi_{\boldsymbol{i}}-\Phi_{\boldsymbol{i}, k,-n}\right)\left(\left(J \frac{\partial \xi_{k}}{\partial x_{j}}\right)_{\boldsymbol{i}}+\left(J \frac{\partial \xi_{k}}{\partial x_{j}}\right)_{\boldsymbol{i}, k,-n}\right)\left(\left(B_{j}\right)_{\boldsymbol{i}}+\left(B_{j}\right)_{\boldsymbol{i}, k,-n}\right)\right] .
\end{aligned}
$$

Substituting the sufficient condition (3.1) into (3.23) yields

$$
\begin{aligned}
I_{1}= & +\frac{1}{2}\left(\boldsymbol{V}_{\boldsymbol{i}}+\boldsymbol{V}_{\boldsymbol{i}, k,+n}\right)^{\mathrm{T}} \widetilde{\mathcal{F}}_{k}\left(\boldsymbol{U}_{\boldsymbol{i}}, \boldsymbol{U}_{\boldsymbol{i}, k,+n},\left(J \frac{\partial \xi_{k}}{\partial \zeta}\right)_{\boldsymbol{i}},\left(J \frac{\partial \xi_{k}}{\partial \zeta}\right)_{\boldsymbol{i}, k,+n}\right) \\
& -\frac{1}{2}\left(\boldsymbol{V}_{\boldsymbol{i}}+\boldsymbol{V}_{\boldsymbol{i}, k,-n}\right)^{\mathrm{T}} \widetilde{\mathcal{F}}_{k}\left(\boldsymbol{U}_{\boldsymbol{i}}, \boldsymbol{U}_{\boldsymbol{i}, k,-n},\left(J \frac{\partial \xi_{k}}{\partial \zeta}\right)_{\boldsymbol{i}},\left(J \frac{\partial \xi_{k}}{\partial \zeta}\right)_{\boldsymbol{i}, k,-n}\right) \\
& -\frac{1}{4}\left[\left(\phi_{\boldsymbol{i}, k,+n}-\phi_{\boldsymbol{i}}\right)\left(\left(J \frac{\partial \xi_{k}}{\partial t}\right)_{\boldsymbol{i}}+\left(J \frac{\partial \xi_{k}}{\partial t}\right)_{\boldsymbol{i}, k,+n}\right)\right.
\end{aligned}
$$




$$
\begin{aligned}
& \left.+\sum_{j=1}^{3}\left(\left(\psi_{j}\right)_{\boldsymbol{i}, k,+n}-\left(\psi_{j}\right)_{\boldsymbol{i}}\right)\left(\left(J \frac{\partial \xi_{k}}{\partial x_{j}}\right)_{\boldsymbol{i}}+\left(J \frac{\partial \xi_{k}}{\partial x_{j}}\right)_{\boldsymbol{i}, k,+n}\right)\right] \\
& +\frac{1}{8}\left(\Phi_{\boldsymbol{i}, k,+n}-\Phi_{\boldsymbol{i}}\right)\left(\left(J \frac{\partial \xi_{k}}{\partial x_{j}}\right)_{\boldsymbol{i}}+\left(J \frac{\partial \xi_{k}}{\partial x_{j}}\right)_{\boldsymbol{i}, k,+n}\right)\left(\left(B_{j}\right)_{\boldsymbol{i}}+\left(B_{j}\right)_{\boldsymbol{i}, k,+n}\right) \\
& -\frac{1}{4}\left[\left(\phi_{\boldsymbol{i}}-\phi_{\boldsymbol{i}, k,-n}\right)\left(\left(J \frac{\partial \xi_{k}}{\partial t}\right)_{\boldsymbol{i}}+\left(J \frac{\partial \xi_{k}}{\partial t}\right)_{\boldsymbol{i}, k,-n}\right)\right. \\
& \left.+\sum_{j=1}^{3}\left(\left(\psi_{j}\right)_{\boldsymbol{i}}-\left(\psi_{j}\right)_{\boldsymbol{i}, k,-n}\right)\left(\left(J \frac{\partial \xi_{k}}{\partial x_{j}}\right)_{\boldsymbol{i}}+\left(J \frac{\partial \xi_{k}}{\partial x_{j}}\right)_{\boldsymbol{i}, k,-n}\right)\right] \\
& +\frac{1}{8}\left(\Phi_{\boldsymbol{i}}-\Phi_{\boldsymbol{i}, k,-n}\right)\left(\left(J \frac{\partial \xi_{k}}{\partial x_{j}}\right)_{\boldsymbol{i}}+\left(J \frac{\partial \xi_{k}}{\partial x_{j}}\right)_{\boldsymbol{i}, k,-n}\right)\left(\left(B_{j}\right)_{\boldsymbol{i}}+\left(B_{j}\right)_{\boldsymbol{i}, k,-n}\right) .
\end{aligned}
$$

Combining (3.24)-(3.27) with (3.21) gives

$$
\begin{aligned}
& I_{1}-I_{2}-I_{3}+I_{4}=+\frac{1}{2}\left(\boldsymbol{V}_{\boldsymbol{i}}+\boldsymbol{V}_{\boldsymbol{i}, k,+n}\right)^{\mathrm{T}} \widetilde{\mathcal{F}}_{k}\left(\boldsymbol{U}_{\boldsymbol{i}}, \boldsymbol{U}_{\boldsymbol{i}, k,+n},\left(J \frac{\partial \xi_{k}}{\partial \zeta}\right)_{\boldsymbol{i}},\left(J \frac{\partial \xi_{k}}{\partial \zeta}\right)_{\boldsymbol{i}, k,+n}\right) \\
& -\frac{1}{2}\left(\boldsymbol{V}_{\boldsymbol{i}, k,-n}+\boldsymbol{V}_{\boldsymbol{i}}\right)^{\mathrm{T}} \widetilde{\mathcal{F}_{k}}\left(\boldsymbol{U}_{\boldsymbol{i}}, \boldsymbol{U}_{\boldsymbol{i}, k,-n},\left(J \frac{\partial \xi_{k}}{\partial \zeta}\right)_{\boldsymbol{i}},\left(J \frac{\partial \xi_{k}}{\partial \zeta}\right)_{\boldsymbol{i}, k,-n}\right) \\
& -\frac{1}{4}\left(\phi_{i}+\phi_{i, k,+n}\right)\left(\left(J \frac{\partial \xi_{k}}{\partial t}\right)_{i}+\left(J \frac{\partial \xi_{k}}{\partial t}\right)_{i, k,+n}\right) \\
& +\frac{1}{4}\left(\phi_{\boldsymbol{i}}+\phi_{\boldsymbol{i}, k,-n}\right)\left(\left(J \frac{\partial \xi_{k}}{\partial t}\right)_{\boldsymbol{i}}+\left(J \frac{\partial \xi_{k}}{\partial t}\right)_{\boldsymbol{i}, k,-n}\right) \\
& -\sum_{j=1}^{3}\left[\frac{1}{4}\left(\left(\psi_{j}\right)_{i}+\left(\psi_{j}\right)_{i, k,+n}\right)\left(\left(J \frac{\partial \xi_{k}}{\partial x_{j}}\right)_{\boldsymbol{i}}+\left(J \frac{\partial \xi_{k}}{\partial x_{j}}\right)_{\boldsymbol{i}, k,+n}\right)\right. \\
& \left.-\frac{1}{4}\left(\left(\psi_{j}\right)_{i}+\left(\psi_{j}\right)_{i, k,-n}\right)\left(\left(J \frac{\partial \xi_{k}}{\partial x_{j}}\right)_{i}+\left(J \frac{\partial \xi_{k}}{\partial x_{j}}\right)_{i, k,-n}\right)\right] \\
& +\sum_{j=1}^{3}\left[\frac{1}{8}\left(\Phi_{\boldsymbol{i}}+\Phi_{\boldsymbol{i}, k,+n}\right)\left(\left(J \frac{\partial \xi_{k}}{\partial x_{j}}\right)_{\boldsymbol{i}}+\left(J \frac{\partial \xi_{k}}{\partial x_{j}}\right)_{\boldsymbol{i}, k,+n}\right)\left(\left(B_{j}\right)_{\boldsymbol{i}}+\left(B_{j}\right)_{\boldsymbol{i}, k,+n}\right)\right. \\
& \left.-\frac{1}{8}\left(\Phi_{\boldsymbol{i}}+\Phi_{\boldsymbol{i}, k,-n}\right)\left(\left(J \frac{\partial \xi_{k}}{\partial x_{j}}\right)_{\boldsymbol{i}}+\left(J \frac{\partial \xi_{k}}{\partial x_{j}}\right)_{\boldsymbol{i}, k,-n}\right)\left(\left(B_{j}\right)_{\boldsymbol{i}}+\left(B_{j}\right)_{\boldsymbol{i}, k,-n}\right)\right] \\
& =\widetilde{\mathfrak{q}_{k}}\left(\boldsymbol{U}_{\boldsymbol{i}}, \boldsymbol{U}_{\boldsymbol{i}, k,+n},\left(J \frac{\partial \xi_{k}}{\partial \zeta}\right)_{\boldsymbol{i}},\left(J \frac{\partial \xi_{k}}{\partial \zeta}\right)_{\boldsymbol{i}, k,+n}\right)-\widetilde{\mathfrak{q}_{k}}\left(\boldsymbol{U}_{\boldsymbol{i}}, \boldsymbol{U}_{\boldsymbol{i}, k,-n},\left(J \frac{\partial \xi_{k}}{\partial \zeta}\right)_{\boldsymbol{i}},\left(J \frac{\partial \xi_{k}}{\partial \zeta}\right)_{\boldsymbol{i}, k,-n}\right),
\end{aligned}
$$

thus (3.22) becomes the numerical entropy identity (3.19). Moreover, it is easy to check the consistency of the numerical entropy flux $\left(\widetilde{\mathfrak{q}_{k}}\right)_{i, k, \pm \frac{1}{2}}^{2 p t h}$ with $\mathfrak{q}_{k}$. The proof is completed. 


\section{High-order accurate ES schemes}

It is known that for the quasi-linear hyperbolic conservation laws, the entropy identity is available only if the solution is smooth. For the discontinuous solutions, one should consider the entropy inequality. Meanwhile, the EC schemes may produce serious nonphysical oscillations near the discontinuities. Those motivate us to construct the high-order accurate ES schemes (satisfying the entropy inequality for the given entropy pair). It can be achieved by adding suitable high-order dissipation to the EC flux (3.6) to obtain the $w$ th-order $(w=2 p-1 \geq 3)$ accurate ES flux

$$
\left(\widehat{\mathcal{F}}_{k}\right)_{i, k,+\frac{1}{2}}^{w \text { th }}=\left(\widetilde{\mathcal{F}_{k}}\right)_{i, k,+\frac{1}{2}}^{2 p \text { th }}-\frac{1}{2} \boldsymbol{D}_{\boldsymbol{i}, k,+\frac{1}{2}} \boldsymbol{Y}_{\boldsymbol{i}, k,+\frac{1}{2}}\langle\langle\widetilde{\boldsymbol{V}}\rangle\rangle_{\boldsymbol{i}, k,+\frac{1}{2}}^{\text {WENO }}
$$

where the matrix $\boldsymbol{D}_{\boldsymbol{i}, k,+\frac{1}{2}}$ is obtained by evaluating $\boldsymbol{D}:=\widehat{\lambda} \boldsymbol{T}^{-1} \boldsymbol{R}(\boldsymbol{T} \boldsymbol{U})$ at $\boldsymbol{i}, k,+\frac{1}{2}$, and $\boldsymbol{T}$ is the "rotational" matrix, which is defined by $\boldsymbol{T}=\operatorname{diag}\left\{1, \boldsymbol{T}_{0}, 1\right\}$ and $\boldsymbol{T}=\operatorname{diag}\left\{1, \boldsymbol{T}_{0}, 1, \boldsymbol{T}_{0}\right\}$ in the RHD and RMHD case, respectively, with

$$
\begin{aligned}
& \boldsymbol{T}_{0}=\left[\begin{array}{ccc}
\cos \varphi \cos \theta & \cos \varphi \sin \theta & \sin \varphi \\
-\sin \theta & \cos \theta & 0 \\
-\sin \varphi \cos \theta & -\sin \varphi \sin \theta & \cos \varphi
\end{array}\right] \\
& \theta=\arctan \left(\left(J \frac{\partial \xi_{k}}{\partial x_{2}}\right) /\left(J \frac{\partial \xi_{k}}{\partial x_{1}}\right)\right) \\
& \varphi=\arctan \left(\left(J \frac{\partial \xi_{k}}{\partial x_{3}}\right) / \sqrt{\left(J \frac{\partial \xi_{k}}{\partial x_{1}}\right)^{2}+\left(J \frac{\partial \xi_{k}}{\partial x_{2}}\right)^{2}}\right) .
\end{aligned}
$$

Here $\hat{\lambda}$ is taken as the spectral radius

$$
\widehat{\lambda}:=\max _{m}\left\{\left|J \frac{\partial \xi_{k}}{\partial t}+L_{k} \lambda_{m}(\boldsymbol{T} \boldsymbol{U})\right|\right\}
$$

with $L_{k}=\sqrt{\sum_{j=1}^{3}\left(J \frac{\partial \xi_{k}}{\partial x_{j}}\right)^{2}}$, and $\boldsymbol{R}$ is a set of scaled eigenvectors such that

$$
\frac{\partial \boldsymbol{U}}{\partial \boldsymbol{V}}=\boldsymbol{R} \boldsymbol{R}^{\mathrm{T}}, \frac{\partial \boldsymbol{F}_{1}}{\partial \boldsymbol{U}}=\boldsymbol{R} \boldsymbol{\Lambda} \boldsymbol{R}^{-1}, \boldsymbol{\Lambda}=\operatorname{diag}\left\{\lambda_{1}, \ldots, \lambda_{m}\right\},
$$

where $\lambda_{1}, \cdots, \lambda_{m}$ are the eigenvalues and $m$ is the equation number (e.g. $m=5$ and 8 for the RHD and RMHD cases respectively when $d=3$ ). The detailed computation of the eigenvalues and eigenvectors has been given in $[21,20]$. To obtain high-order accuracy, the high-order WENO reconstruction is performed in the scaled entropy variables. More specifically, the $w$ th-order $(w=$ $2 p-1)$ WENO reconstruction [36] is performed on $\left\{\widetilde{\boldsymbol{V}}=\boldsymbol{R}_{\boldsymbol{i}, k,+\frac{1}{2}}^{\mathrm{T}}(\boldsymbol{T} \boldsymbol{U}) \boldsymbol{T}_{\boldsymbol{i}, k,+\frac{1}{2}} \boldsymbol{V}\right\}$ in the $i_{k}$-direction to obtain the left and right limit values denoted by $\widetilde{\boldsymbol{V}}_{\boldsymbol{i}, k,+\frac{1}{2}}^{\mathrm{WENO},-}$ and $\widetilde{\boldsymbol{V}}_{\boldsymbol{i}, k,+\frac{1}{2}}^{\mathrm{WENO},+}$, and then define

$$
\langle\langle\widetilde{\boldsymbol{V}}\rangle\rangle_{\boldsymbol{i}, k,+\frac{1}{2}}^{\mathrm{WENO}}=\widetilde{\boldsymbol{V}}_{\boldsymbol{i}, k,+\frac{1}{2}}^{\mathrm{WENO},+}-\widetilde{\boldsymbol{V}}_{\boldsymbol{i}, k,+\frac{1}{2}}^{\mathrm{WENO},-} .
$$


In (4.1), the diagonal matrix $\boldsymbol{Y}_{\boldsymbol{i}, k,+\frac{1}{2}}$ is used to enforce the "sign" property, see [5], with the diagonal component given by

$$
\left(\boldsymbol{Y}_{\boldsymbol{i}, k,+\frac{1}{2}}\right)_{l, l}= \begin{cases}1, & \left.\operatorname{sign}\left(\left\langle\widetilde{\boldsymbol{V}}_{l}\right\rangle\right\rangle_{\boldsymbol{i}, k,+\frac{1}{2}}\right)=\operatorname{sign}\left(\llbracket \tilde{\boldsymbol{V}}_{l} \rrbracket_{\boldsymbol{i}, k,+\frac{1}{2}}\right), \\ 0, & \text { otherwise }\end{cases}
$$

where $\llbracket a \rrbracket_{i, k,+\frac{1}{2}}=a_{i, k,+1}-a_{\boldsymbol{i}}$.

Theorem 4.1. By replacing the $2 p$ th-order EC flux with wth-order ES flux (4.1), the following schemes

$$
\begin{aligned}
\frac{\mathrm{d}}{\mathrm{d} t} \mathcal{U}_{\boldsymbol{i}} & =-\sum_{k=1}^{3} \frac{1}{\Delta \xi_{k}}\left(\left(\widehat{\mathcal{F}_{k}}\right)_{\boldsymbol{i}, k,+\frac{1}{2}}^{w \mathrm{th}}-\left(\widehat{\mathcal{F}_{k}}\right)_{\boldsymbol{i}, k,-\frac{1}{2}}^{w \mathrm{th}}\right)-\Phi^{\prime}\left(\boldsymbol{V}_{\boldsymbol{i}}\right)^{\mathrm{T}} \sum_{k=1}^{3} \frac{1}{\Delta \xi_{k}}\left(\left(\widetilde{\mathcal{B}_{k}}\right)_{\boldsymbol{i}, k,+\frac{1}{2}}^{2 p \text { th }}-\left(\widetilde{\mathcal{B}_{k}}\right)_{\boldsymbol{i}, k,-\frac{1}{2}}^{2 p \text { th }}\right), \\
\frac{\mathrm{d}}{\mathrm{d} t} J_{\boldsymbol{i}} & \left.=-\sum_{k=1}^{3} \frac{1}{\Delta \xi_{k}}\left(\widetilde{J \frac{\partial \xi_{k}}{\partial t}}\right)_{\boldsymbol{i}, k,+\frac{1}{2}}^{2 p \mathrm{th}}-\left(\widetilde{J \frac{\partial \xi_{k}}{\partial t}}\right)_{i, k,-\frac{1}{2}}^{2 p \mathrm{th}}\right),
\end{aligned}
$$

are ES. Specially, they satisfy the entropy inequality

$$
\frac{\mathrm{d}}{\mathrm{d} t} J_{\boldsymbol{i}} \eta\left(\boldsymbol{U}_{\boldsymbol{i}}(t)\right)+\sum_{k=1}^{3} \frac{1}{\Delta \xi_{k}}\left(\left(\widehat{\mathfrak{q}_{k}}\right)_{\boldsymbol{i}, k,+\frac{1}{2}}^{w \mathrm{th}}-\left(\widehat{\mathfrak{q}_{k}}\right)_{i, k,-\frac{1}{2}}^{w \mathrm{th}}\right) \leqslant 0,
$$

with the consistent numerical entropy fluxes

$$
\left(\widehat{\mathfrak{q}_{k}}\right)_{\boldsymbol{i}, k,+\frac{1}{2}}^{\text {th }}=\left(\widetilde{\mathfrak{q}_{k}}\right)_{i, k,+\frac{1}{2}}^{2 p \text { th }}-\frac{1}{2} \widehat{\lambda}_{i, k,+\frac{1}{2}}\{\tilde{\boldsymbol{V}}\}_{\boldsymbol{i}, k,+\frac{1}{2}}^{\mathrm{T}} \boldsymbol{Y}_{\boldsymbol{i}, k,+\frac{1}{2}}\langle\langle\widetilde{\boldsymbol{V}}\rangle\rangle_{\boldsymbol{i}, k,+\frac{1}{2}}^{\text {WENO }},
$$

where $\left\{\{a\}_{i, k,+\frac{1}{2}}=\frac{1}{2}\left(a_{\boldsymbol{i}, k,+1}+a_{\boldsymbol{i}}\right)\right.$.

Proof. Taking the dot product of $\boldsymbol{V}_{\boldsymbol{i}}$ and (4.2) gives

$$
\begin{aligned}
& \frac{\mathrm{d}}{\mathrm{d} t}\left(J_{\boldsymbol{i}} \eta_{\boldsymbol{i}}\right)=-\sum_{k=1}^{3} \frac{1}{\Delta \xi_{k}}\left(\left(\widetilde{\mathfrak{q}_{k}}\right)_{\boldsymbol{i}, k,+\frac{1}{2}}^{2 p \mathrm{th}}-\left(\widetilde{\mathfrak{q}_{k}}\right)_{\boldsymbol{i}, k,-\frac{1}{2}}^{2 p \mathrm{th}}\right) \\
& +\sum_{k=1}^{3} \frac{1}{2 \Delta \xi_{k}}\left(\widehat{\lambda}_{\boldsymbol{i}, k,+\frac{1}{2}} \boldsymbol{V}_{i}^{\mathrm{T}} \boldsymbol{T}_{\boldsymbol{i}, k,+\frac{1}{2}}^{-1} \boldsymbol{R}_{\boldsymbol{i}, k,+\frac{1}{2}}(\boldsymbol{T} \boldsymbol{U}) \boldsymbol{Y}_{\boldsymbol{i}, k,+\frac{1}{2}}\langle\langle\tilde{\boldsymbol{V}}\rangle\rangle_{\boldsymbol{i}, k,+\frac{1}{2}}^{\mathrm{WENO}}\right. \\
& \left.-\widehat{\lambda}_{\boldsymbol{i}, k,-\frac{1}{2}} \boldsymbol{V}_{i}^{\mathrm{T}} \boldsymbol{T}_{\boldsymbol{i}, k,-\frac{1}{2}}^{-1} \boldsymbol{R}_{\boldsymbol{i}, k,-\frac{1}{2}}(\boldsymbol{T} \boldsymbol{U}) \boldsymbol{Y}_{\boldsymbol{i}, k,-\frac{1}{2}}\langle\langle\tilde{\boldsymbol{V}}\rangle\rangle_{\boldsymbol{i}, k,-\frac{1}{2}}^{\mathrm{WENO}}\right) \\
& =-\sum_{k=1}^{3} \frac{1}{\Delta \xi_{k}}\left(\left(\widehat{\mathfrak{q}_{k}}\right)_{\boldsymbol{i}, k,+\frac{1}{2}}^{w \mathrm{th}}-\left(\widehat{\mathfrak{q}_{k}}\right)_{i, k,-\frac{1}{2}}^{w \mathrm{th}}\right) \\
& -\sum_{k=1}^{3} \frac{1}{4 \Delta \xi_{k}}\left(\widehat{\lambda}_{\boldsymbol{i}, k,+\frac{1}{2}} \llbracket \boldsymbol{V} \rrbracket_{\boldsymbol{i}, k,+\frac{1}{2}}^{\mathrm{T}} \boldsymbol{T}_{\boldsymbol{i}, k,+\frac{1}{2}}^{-1} \boldsymbol{R}_{\boldsymbol{i}, k,+\frac{1}{2}}(\boldsymbol{T} \boldsymbol{U}) \boldsymbol{Y}_{\boldsymbol{i}, k,+\frac{1}{2}}\langle\langle\tilde{\boldsymbol{V}}\rangle\rangle_{\boldsymbol{i}, k,+\frac{1}{2}}^{\mathrm{WENO}}\right. \\
& \left.+\widehat{\lambda}_{\boldsymbol{i}, k,-\frac{1}{2}} \llbracket \boldsymbol{V} \rrbracket_{\boldsymbol{i}, k,-\frac{1}{2}}^{\mathrm{T}} \boldsymbol{T}_{\boldsymbol{i}, k,-\frac{1}{2}}^{-1} \boldsymbol{R}_{\boldsymbol{i}, k,-\frac{1}{2}}(\boldsymbol{T} \boldsymbol{U}) \boldsymbol{Y}_{\boldsymbol{i}, k,-\frac{1}{2}}\langle\langle\tilde{\boldsymbol{V}}\rangle\rangle_{\boldsymbol{i}, k,-\frac{1}{2}}^{\mathrm{WENO}}\right)
\end{aligned}
$$




$$
\begin{aligned}
= & -\sum_{k=1}^{3} \frac{1}{\Delta \xi_{k}}\left(\left(\widehat{\mathfrak{q}_{k}}\right)_{i, k,+\frac{1}{2}}^{w \mathrm{th}}-\left(\widehat{\mathfrak{q}_{k}}\right)_{\boldsymbol{i}, k,-\frac{1}{2}}^{w \mathrm{th}}\right) \\
& -\sum_{k=1}^{3} \frac{1}{4 \Delta \xi_{k}}\left(\widehat{\lambda}_{\boldsymbol{i}, k,+\frac{1}{2}} \llbracket \widetilde{\boldsymbol{V}} \rrbracket_{\boldsymbol{i}, k,+\frac{1}{2}}^{\mathrm{T}} \boldsymbol{Y}_{\boldsymbol{i}, k,+\frac{1}{2}}\langle\langle\widetilde{\boldsymbol{V}}\rangle\rangle_{\boldsymbol{i}, k,+\frac{1}{2}}^{\mathrm{WENO}}+\widehat{\lambda}_{\boldsymbol{i}, k,-\frac{1}{2}} \llbracket \widetilde{\boldsymbol{V}} \rrbracket_{\boldsymbol{i}, k,-\frac{1}{2}}^{\mathrm{T}} \boldsymbol{Y}_{\boldsymbol{i}, k,-\frac{1}{2}}\langle\langle\widetilde{\boldsymbol{V}}\rangle\rangle_{\boldsymbol{i}, k,-\frac{1}{2}}^{\mathrm{WENO}}\right),
\end{aligned}
$$

where the 1st equality uses the entropy identity satisfied by the $2 p$ th-order EC scheme (3.19), the 2nd equality uses (4.4). From the definition of $\boldsymbol{Y}_{\boldsymbol{i}, k, \pm \frac{1}{2}}$, one can get

$$
\llbracket \widetilde{\boldsymbol{V}} \rrbracket_{\boldsymbol{i}, k, \pm \frac{1}{2}}^{\mathrm{T}} \boldsymbol{Y}_{\boldsymbol{i}, k, \pm \frac{1}{2}}\langle\langle\tilde{\boldsymbol{V}}\rangle\rangle_{\boldsymbol{i}, k, \pm \frac{1}{2}}^{\mathrm{WENO}} \geqslant 0
$$

therefore, it holds

$$
\frac{\mathrm{d}}{\mathrm{d} t} J_{\boldsymbol{i}} \eta\left(\boldsymbol{U}_{\boldsymbol{i}}(t)\right)+\sum_{k=1}^{3} \frac{1}{\Delta \xi_{k}}\left(\left(\widehat{\mathfrak{q}_{k}}\right)_{\boldsymbol{i}, k,+\frac{1}{2}}^{w \mathrm{th}}-\left(\widehat{\mathfrak{q}_{k}}\right)_{\boldsymbol{i}, k,-\frac{1}{2}}^{w \mathrm{th}}\right) \leqslant 0 .
$$

Remark 4.1. When the solution is a constant state, the dissipation terms vanish, so that the ES schemes preserve the free-stream state.

\section{Adaptive moving mesh strategy}

This section presents our adaptive moving mesh strategy at time $t=t^{n}$ for the completeness of the paper, but focuses on the mesh iteration redistribution with the solution obtained by the finite difference scheme. It is similar to that used in [21], where the mesh iteration redistribution depends on the solution obtained by the second-order accurate finite volume scheme. Unless otherwise stated, the dependence of the variables on $t$ will be omitted.

Consider the mesh adaption functional

$$
\widetilde{E}(\boldsymbol{x})=\frac{1}{2} \sum_{k=1}^{3} \int_{\Omega_{l}}\left(\nabla_{\boldsymbol{\xi}} x_{k}\right)^{\mathrm{T}} \boldsymbol{G}_{k}\left(\nabla_{\boldsymbol{\xi}} x_{k}\right) \mathrm{d} \boldsymbol{\xi},
$$

where $\boldsymbol{G}_{k}$ is the given symmetric positive definite matrix, depending on the solution $\boldsymbol{U}$. Solving the Euler-Lagrange equations of (5.1)

$$
\nabla_{\boldsymbol{\xi}} \cdot\left(\boldsymbol{G}_{k} \nabla_{\boldsymbol{\xi}} x_{k}\right)=0, \boldsymbol{\xi} \in \Omega_{c}, k=1,2,3
$$

will give directly a coordinate transformation $\boldsymbol{x}=\boldsymbol{x}(\boldsymbol{\xi})$ from the computational domain $\Omega_{c}$ to the physical domain $\Omega_{p}$. The concentration of the mesh points is controlled by $\boldsymbol{G}_{k}$, which in general depends on the solutions or their derivatives of the underlying governing equations and is one of the most important elements in the adaptive moving mesh method. Different problems may be equipped with different $\boldsymbol{G}_{k}$. For example, the Winslow variable diffusion method [62] is considering the simplest choice of $\boldsymbol{G}_{k}$ defined by

$$
\boldsymbol{G}_{k}=\omega \boldsymbol{I}_{3}
$$


where $\omega$ is a positive weight function, called the monitor function, and may be taken as

$$
\omega=\sqrt{1+\alpha\left|\nabla_{\boldsymbol{\xi}} \sigma\right| / \max \left|\nabla_{\boldsymbol{\xi}} \sigma\right|}
$$

here $\sigma$ is some physical variable and $\alpha$ is a positive parameter. There are several other choices of the monitor functions, see $[7,29,31,58,59]$.

Remark 5.1. The monitor function is computed from the solutions of the underlying physical equations (2.8), thus is not smooth in general. To get a smoother (adaptive) mesh, the following low pass filter

$$
\omega_{i_{1}, i_{2}, i_{3}} \leftarrow \sum_{j_{1}, j_{2}, j_{3}=0, \pm 1}\left(\frac{1}{2}\right)^{\left|j_{1}\right|+\left|j_{2}\right|+\left|j_{3}\right|+3} \omega_{i_{1}+j_{1}, i_{2}+j_{2}, i_{3}+j_{3}}
$$

is applied $3 \sim 5$ times in this work.

The mesh equations (5.2) are approximated by the central difference scheme on the computational mesh and then solved by using the Jacobi iteration method

$$
\sum_{k=1}^{3}\left[\left(\omega_{\boldsymbol{i}}+\omega_{i, k,+1}\right)\left(\boldsymbol{x}_{\boldsymbol{i}, k,+1}^{[\nu]}-\boldsymbol{x}_{\boldsymbol{i}}^{[\nu+1]}\right)-\left(\omega_{\boldsymbol{i}}+\omega_{i, k,-1}\right)\left(\boldsymbol{x}_{\boldsymbol{i}}^{[\nu+1]}-\boldsymbol{x}_{\boldsymbol{i}, k,-1}^{[\nu]}\right)\right]=0, \nu=0,1, \cdots, \mu,
$$

in parallel, where $\boldsymbol{x}_{\boldsymbol{i}}^{[0]}:=\boldsymbol{x}_{\boldsymbol{i}}^{n}$, and $\omega$ is computed by using the solution $\boldsymbol{U}$ at $t^{n}$. In our numerical tests, the total iteration number $\mu$ is taken as 10 , unless otherwise stated.

Once the mesh $\left\{\boldsymbol{x}_{\boldsymbol{i}}^{[\mu]}\right\}$ is obtained, the final adaptive mesh is given by

$$
\boldsymbol{x}_{\boldsymbol{i}}^{n+1}:=\boldsymbol{x}_{\boldsymbol{i}}^{n}+\Delta_{\tau}\left(\delta_{\tau} \boldsymbol{x}\right)_{i}^{n},\left(\delta_{\tau} \boldsymbol{x}\right)_{i}^{n}:=\boldsymbol{x}_{\boldsymbol{i}}^{[\mu]}-\boldsymbol{x}_{\boldsymbol{i}}^{n}
$$

where the parameter $\Delta_{\tau}$ is used to limit the mesh point movement

$$
\Delta_{\tau} \leqslant \begin{cases}-\frac{1}{2\left(\delta_{\tau} x_{k}\right)_{i}}\left[\left(x_{k}\right)_{i}^{n}-\left(x_{1}\right)_{i, k,-1}^{n}\right], & \left(\delta_{\tau} x_{k}\right)_{i}<0 \\ +\frac{1}{2\left(\delta_{\tau} x_{k}\right)_{i}}\left[\left(x_{k}\right)_{i, k,+1}^{n}-\left(x_{1}\right)_{\boldsymbol{i}}^{n}\right], & \left(\delta_{\tau} x_{k}\right)_{\boldsymbol{i}}>0 .\end{cases}
$$

Finally, the mesh velocity in (3.18) is defined by $\dot{\boldsymbol{x}}_{\boldsymbol{i}}^{n}:=\Delta_{\tau}\left(\delta_{\tau} \boldsymbol{x}\right)_{i}^{n} / \Delta t^{n}$, where the time step size $\Delta t^{n}$ is determined by $(6.1)$.

\section{Numerical results}

This section conducts several 2D and 3D numerical tests in the RHDs and RMHDs to validate the convergence orders of our sixth-order accurate EC schemes on moving meshes (denoted by MM-06), and the convergence orders and the shock-capturing ability of our fifth-order accurate ES schemes on moving meshes (denoted by MM-05). The numerical results are also compared to those 
obtained by the fifth-order accurate ES schemes on the static uniform mesh (denoted by UM-05) [19], and the second-order accurate ES adaptive moving mesh schemes (denoted by MM-02) [21]. Our schemes are implemented in parallel based on the data structure of the PLUTO code [48], and all simulations are performed with the CPU nodes of the High-performance Computing Platform of Peking University (Linux Red Hat environment, two Intel Xeon E5-2697A V4 (16 cores ×2) per node, and core frequency of $2.6 \mathrm{GHz}$ ). Unless otherwise stated, the adiabatic index $\Gamma$ is taken as $5 / 3$ and the time step size $\Delta t^{n}$ is determined by the following CFL condition

$$
\Delta t^{n}=\frac{\mathrm{CFL}}{\sum_{k=1}^{d} \max _{\boldsymbol{i}} \varrho_{k, \boldsymbol{i}}^{n} / \Delta \xi_{k}},
$$

where $\varrho_{k, i}^{n}$ is the spectral radius of $\partial \mathcal{F}_{k} / \partial \mathcal{U}+\Phi^{\prime}(\boldsymbol{V}) \partial \mathcal{B}_{k} / \partial \mathcal{U}$ evaluated at $\boldsymbol{i}$ and $t^{n}$, and the CFL number is taken as 0.4 and 0.3 for the $2 \mathrm{D}$ and $3 \mathrm{D}$ tests, respectively.

\section{1. $2 D$ tests}

Example 6.1 (2D RMHD isentropic vortex problem). It describes a $2 \mathrm{D}$ vortex moving with a constant speed $(-0.5,-0.5)$ and is solved to test the convergence orders and the change of the total entropy. Specifically, the physical domain $\Omega_{p}$ is taken as $[-R, R] \times[-R, R]$ with $R=5$ and periodic boundary conditions. The explicit analytical solutions at time $t$ and the spatial point $\left(x_{1}, x_{2}\right)$ given first in [18] are

$$
\begin{aligned}
\rho & =\left(1-\sigma \exp \left(1-r^{2}\right)\right)^{\frac{1}{\Gamma-1}}, p=\rho^{\Gamma}, \\
\boldsymbol{v} & =\frac{1}{4-2\left(\widetilde{v}_{1}+\widetilde{v}_{2}\right)}\left((2+\sqrt{2}) \widetilde{v}_{1}+(2-\sqrt{2}) \widetilde{v}_{2}-2,(2+\sqrt{2}) \widetilde{v}_{2}+(2-\sqrt{2}) \widetilde{v}_{1}-2,0\right), \\
\boldsymbol{B} & =\frac{1}{2}\left((\sqrt{2}+1) \widetilde{B}_{1}-(\sqrt{2}-1) \widetilde{B}_{2},(\sqrt{2}+1) \widetilde{B}_{2}-(\sqrt{2}-1) \widetilde{B}_{1}, 0\right),
\end{aligned}
$$

where

$$
\begin{aligned}
& \Gamma=5 / 3, \sigma=0.2, B_{0}=0.05, r=\sqrt{\widetilde{x}_{1}^{2}+\widetilde{x}_{2}^{2}}, \\
& \widetilde{x}_{k}=\widehat{x}_{k}+(\sqrt{2}-1)\left(\widehat{x}_{1}+\widehat{x}_{2}\right) / 2, k=1,2, \\
& \left(\widehat{x}_{1}, \widehat{x}_{2}\right)=\left(2 k_{1} R+x_{1}+t / 2-1,2 k_{2} R+x_{2}+t / 2-1\right),\left(\widehat{x}_{1}, \widehat{x}_{2}\right) \in[-R, R] \times[-R, R], k_{1}, k_{2} \in \mathbb{Z}, \\
& \left(\widetilde{v}_{1}, \widetilde{v}_{2}\right)=\left(-\widetilde{x}_{2}, \widetilde{x}_{1}\right) f, f=\sqrt{\frac{\kappa \exp \left(1-r^{2}\right)}{\kappa r^{2} \exp \left(1-r^{2}\right)+(\Gamma-1) \rho+\Gamma p}}, \kappa=2 \Gamma \sigma \rho+(\Gamma-1) B_{0}^{2}\left(2-r^{2}\right), \\
& \left(\widetilde{B}_{1}, \widetilde{B}_{2}\right)=B_{0} \exp \left(1-r^{2}\right)\left(-\widetilde{x}_{2}, \widetilde{x}_{1}\right) .
\end{aligned}
$$

The problem is solved with a series of $N \times N$ meshes until $t=4$. 
First, we test the sixth-order EC scheme on moving meshes (MM-06) with the following moving mesh strategy

$$
\begin{aligned}
& \left(x_{1}\right)_{i_{1}, i_{2}}=\stackrel{\circ}{x}_{1}+0.2 \cos (\pi t / 4) \sin \left(3 \pi \stackrel{\circ}{x}_{2} / R\right),\left(x_{2}\right)_{i_{1}, i_{2}}=\stackrel{\circ}{x}_{2}+0.2 \cos (\pi t / 4) \sin \left(3 \pi \stackrel{\circ}{x}_{1} / R\right), \\
& \stackrel{\circ}{x}_{1}=2 i_{1} R /(N-1), \stackrel{\circ}{x}_{2}=2 i_{2} R /(N-1), i_{1}, i_{2}=0,1, \cdots, N-1 .
\end{aligned}
$$

The time step size is chosen as $\Delta t^{n}=\mathrm{CFL} \Delta \xi_{1}^{2}$ to make the spatial error dominant. Figure 6.1 gives the 10 equally spaced contours of the rest-mass density and the moving meshes with $N=40$ at different times. One can see that the shape of the vortex is preserved well.

Next, the problem is resolved by using the fifth-order ES scheme with the adaptive moving mesh (MM-05) and the following monitor function

$$
\omega=\sqrt{1+20\left|\nabla_{\xi} \rho\right| / \max \left|\nabla_{\xi} \rho\right|+10\left|\Delta_{\xi} \rho\right| / \max \left|\Delta_{\xi} \rho\right|} .
$$

The time step size is chosen as $\Delta t^{n}=\mathrm{CFL} \Delta \xi_{1}^{5 / 3}$ to make the spatial error dominant. Figure 6.2 plots the adaptive meshes of $N=40$ at different times, which show that the concentration of the mesh points follows the propagation of the vortex well.

Figure 6.3 plots corresponding errors in the rest-mass density $\rho$ and convergence orders of MM-06 and MM-05. One can see that MM-06 and MM-05 can achieve sixth- and fifth-order accuracies respectively.

Finally, we examine the EC and ES property of our schemes. Figure 6.4 presents the evolution of the discrete total entropy $\sum_{i_{1}, i_{2}} J_{i_{1}, i_{2}} \eta\left(\boldsymbol{U}_{i_{1}, i_{2}}\right) / N^{2}$ with respect to time obtained by MM-06 and MM-05 with $N=160$. We can see that the total entropy of the EC scheme almost keeps unchanged, while the total entropy of the ES scheme decays as expected.

It should be noted that MM-05 with the moving mesh (6.2) and MM-06 with the adaptive moving mesh and the monitor (6.3) can also respectively get fifth-order and sixth-order. Their results are omitted here due to limited space.

Example 6.2 (RHD Riemann problem I). This example considers the 2D RHD Riemann problem with the initial data

$$
\left(\rho, v_{1}, v_{2}, p\right)= \begin{cases}(0.5,0.5,-0.5,5), & x_{1}>0.5, x_{2}>0.5 \\ (1,0.5,0.5,5), & x_{1}<0.5, x_{2}>0.5 \\ (3,-0.5,0.5,5), & x_{1}<0.5, x_{2}<0.5 \\ (1.5,-0.5,-0.5,5), & x_{1}>0.5, x_{2}<0.5\end{cases}
$$

It describes the interaction of four contact discontinuities (vortex sheets) with the same sign (the negative sign). 


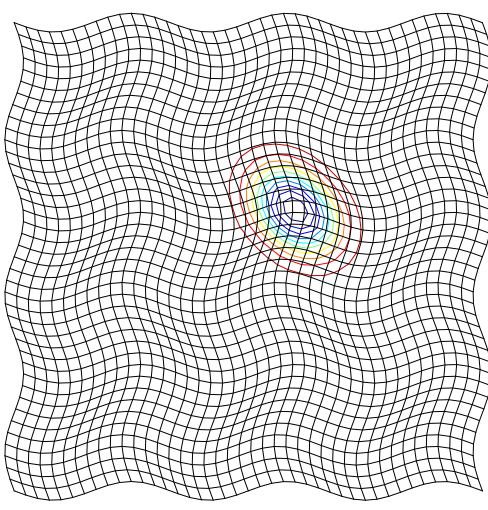

(a) $t=0$

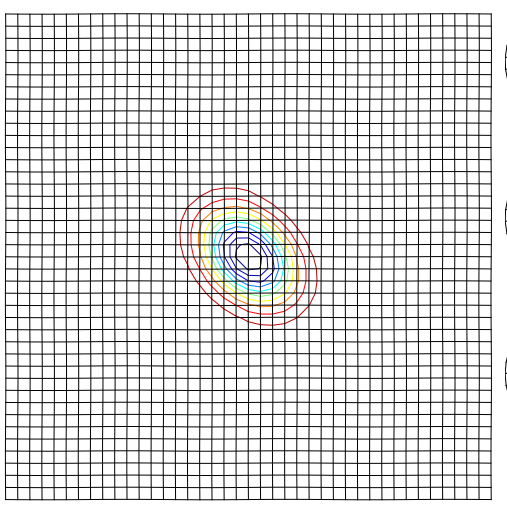

(b) $t=2$

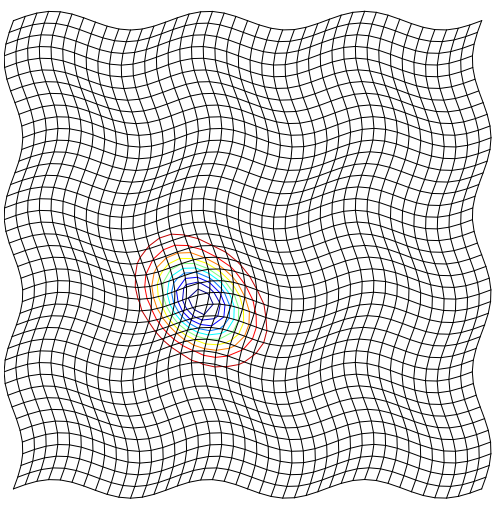

(c) $t=4$

Figure 6.1: Example 6.1: Adaptive meshes and rest-mass density contours at different times obtained by MM-06 with the moving mesh (6.2). $N=40$ and 10 equally spaced contour lines.

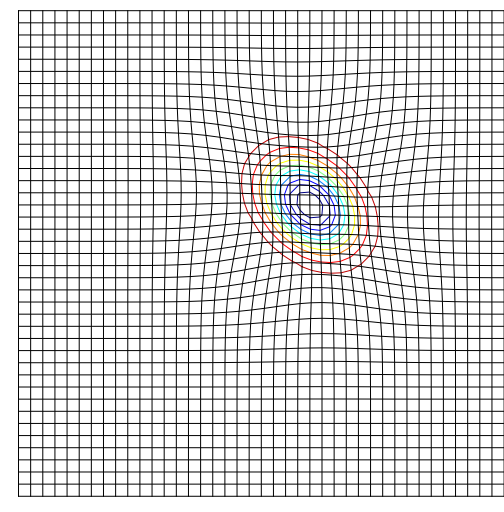

(a) $t=0$

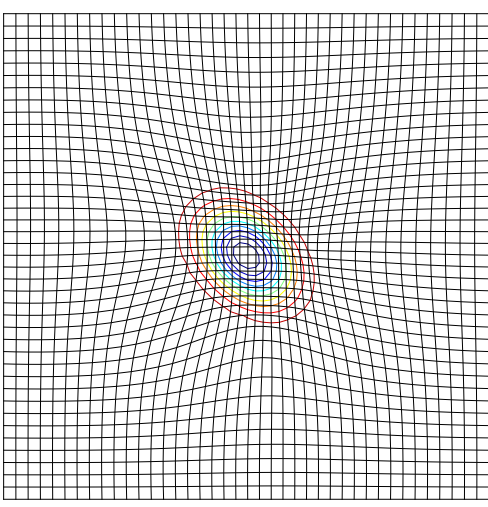

(b) $t=2$

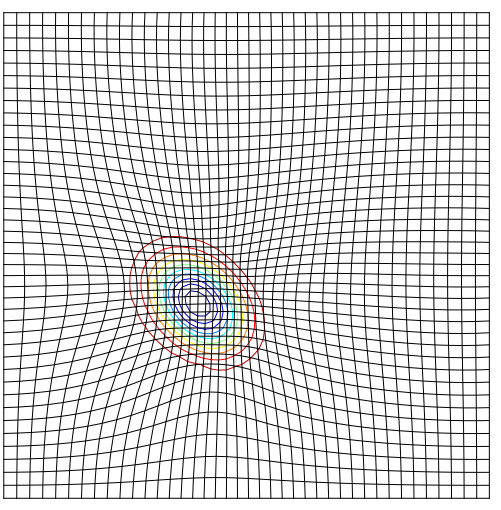

(c) $t=4$

Figure 6.2: Example 6.1: Adaptive meshes and rest-mass density contours at different times obtained by MM-05 with adaptive mesh velocity and the monitor (6.3). $N=40$ and 10 equally spaced contour lines.

The monitor function is chosen as (5.3) with $\alpha=1200$ and $\sigma=\ln \rho$. Figure 6.5 shows the adaptive mesh of MM-05, 40 equally spaced contour lines of $\ln \rho$, and the cut lines of $\ln \rho$ along $x_{2}=x_{1}$ at $t=0.4$ obtained by using our ES schemes with $N \times N$ meshes. As time increases, a spiral with the low rest-mass density around the point $(0.5,0.5)$ emerges, and the adaptive concentration of the mesh points follows the spiral formation well, see Figure 6.5(a), so that some important features are well-captured. Figure $6.5(\mathrm{f})$ shows the solution of MM-05 with $N=200$ is very close to that of UM-05 with $N=500$, and MM-05 does not cause spurious oscillations near $(0.86,0.86)$, see the small box in the upper right corner in Figure 6.5(f). The CPU times (see the parentheses in the captions of Figures 6.5(b) and 6.5(c)) clearly highlight the efficiency of the adaptive moving mesh scheme, since it takes only $17.8 \%$ CPU time of the latter. Figures 6.5(d) and 6.5(e) show that the fifth-order scheme MM-05 gives better results with comparable CPU time than the second-order 


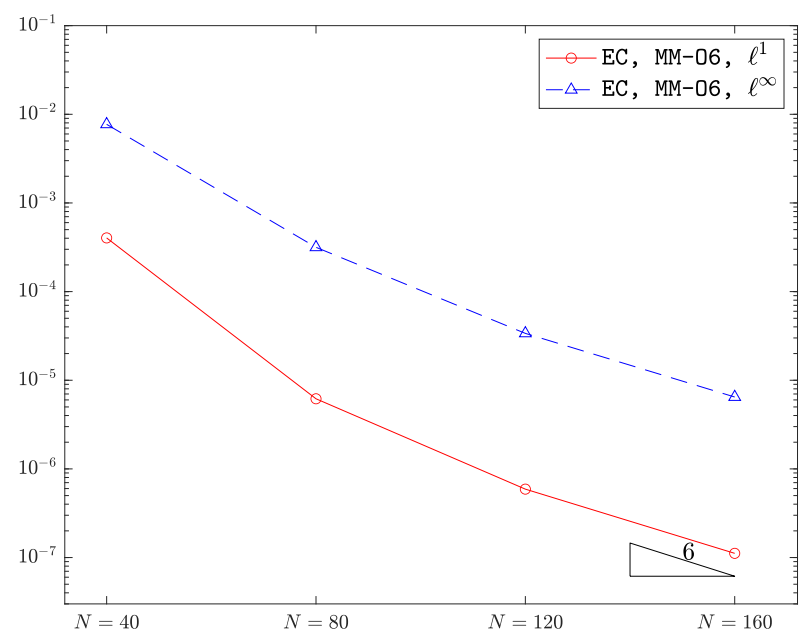

(a) EC, MM-06

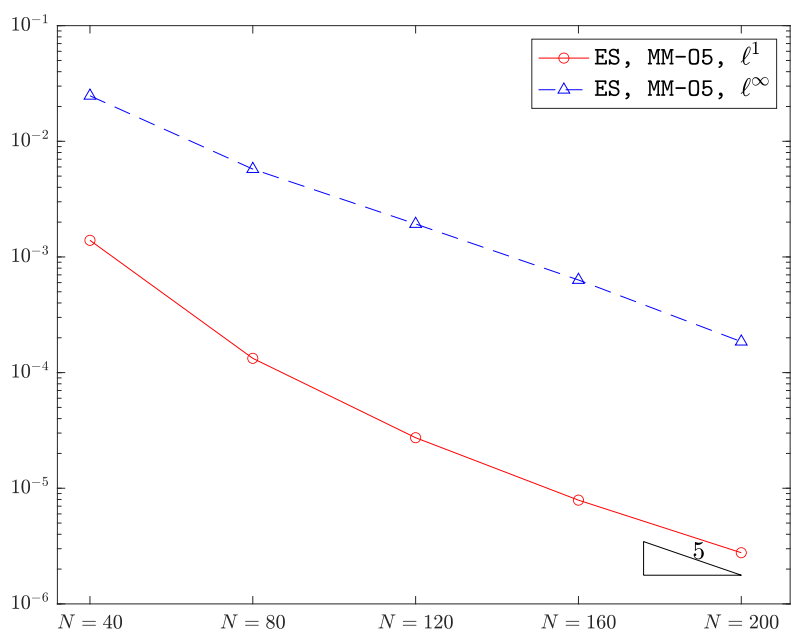

(b) ES, MM-05

Figure 6.3: Example 6.1: The errors and convergence orders in $\rho$ at $t=4$.

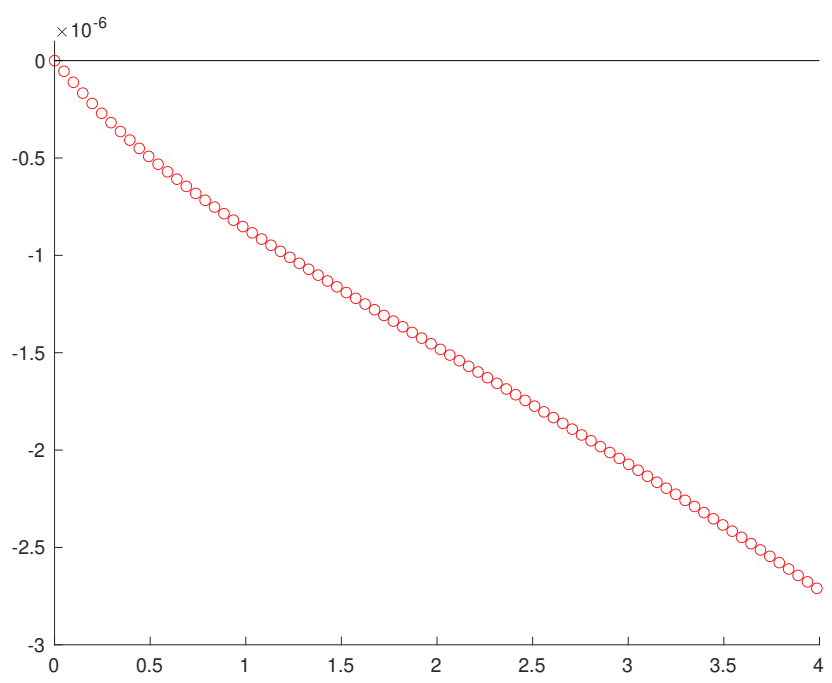

Figure 6.4: Example 6.1: The evolution of the discrete total entropy with $160 \times 160$ meshes. The line and symbols aare obtained by using the EC scheme MM-06, and the ES scheme MM-05, respectively.

scheme MM-02 [21], thus MM-05 outperforms MM-02.

Example 6.3 (RHD Riemann problem II). The initial data of this 2D RHD Riemann problem are

$$
\left(\rho, v_{1}, v_{2}, p\right)= \begin{cases}(1,0,0,1), & x_{1}>0.5, x_{2}>0.5, \\ (0.5771,-0.3529,0,0.4), & x_{1}<0.5, x_{2}>0.5, \\ (1,-0.3529,-0.3529,1), & x_{1}<0.5, x_{2}<0.5, \\ (0.5771,0,-0.3529,0.4), & x_{1}>0.5, x_{2}<0.5,\end{cases}
$$

which is about the interaction of four rarefaction waves. 


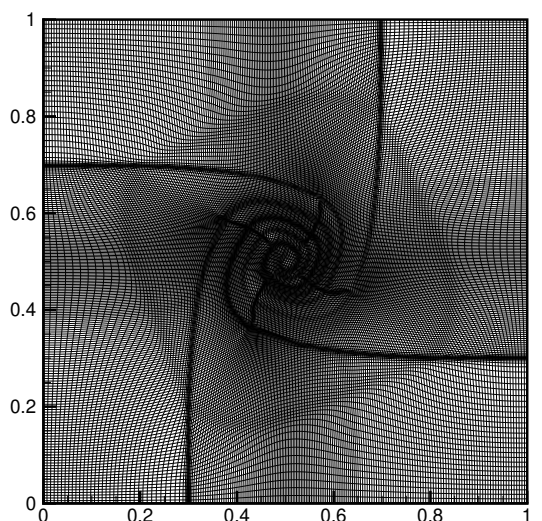

(a) MM-05 with $N=200$

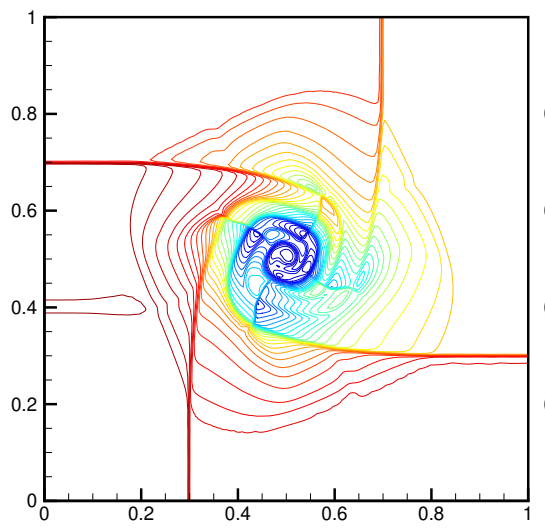

(d) MM-05 with $N=150(29 \mathrm{~s})$

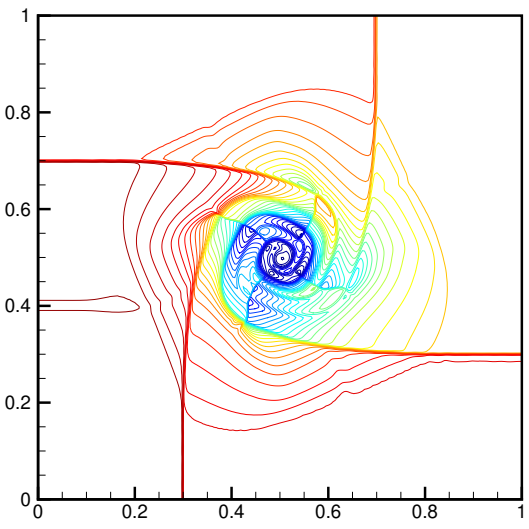

(b) MM-05 with $N=200(1 \mathrm{~m} 02 \mathrm{~s})$

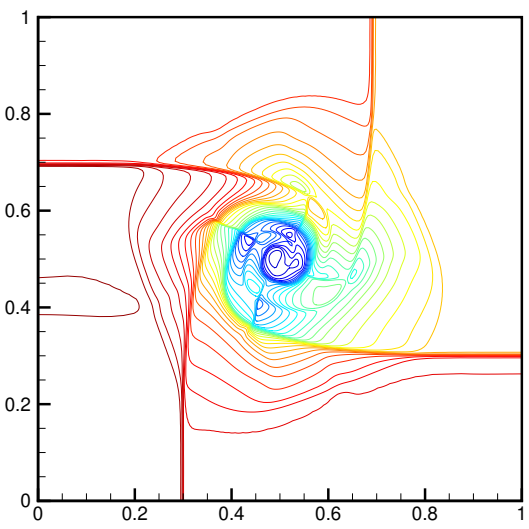

(e) MM-02 with $N=200$ (30s)

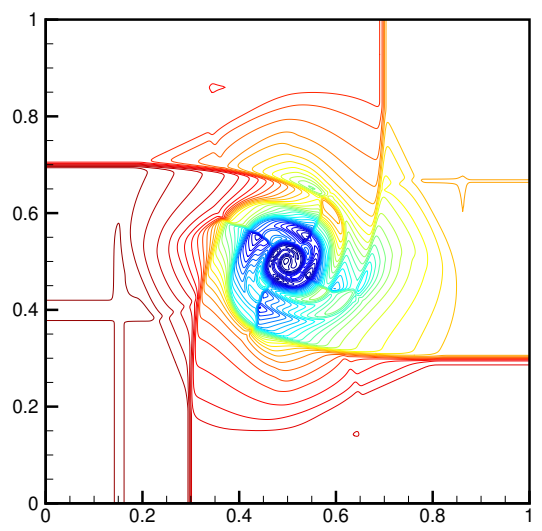

(c) UM-05 with $N=500(5 \mathrm{~m} 49 \mathrm{~s})$

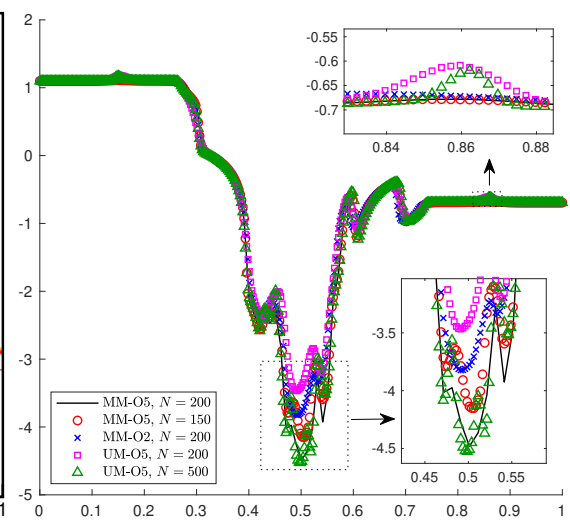

(f) $\ln \rho$ along $x_{2}=x_{1}$

Figure 6.5: Example 6.2. Adaptive mesh of MM-05 with $N=200,40$ equally spaced contour lines of $\ln \rho$, and cut lines of $\ln \rho$ along $x_{2}=x_{1}$ obtained by using ES schemes. CPU times are listed in parentheses.

The monitor function is the same as that in the last example. Figure 6.6 presents the adaptive mesh of MM-05, the contours of the density logarithms $\ln \rho$ with 40 equally spaced lines, and $\ln \rho$ along $x_{2}=x_{1}$ at $t=0.4$. The results show that those four initial discontinuities first evolve as four rarefaction waves and then interact each other and form two (almost parallel) curved shock waves perpendicular to the line $x_{2}=x_{1}$ as time increases. It is seen that the adaptive moving mesh schemes capture the rarefaction waves and the shock waves well. Figure 6.6(f) compares the results of MM-05 with $N=200$ to UM-05 with $N=500$, which are very close to each other, but the former takes about $30.6 \% \mathrm{CPU}$ time. One can also find from Figure $6.6(\mathrm{f})$ that MM-05 with $N=150$ gives better results than MM-02 with $N=200$ when using comparable CPU time.

Example 6.4 (RHD Riemann problem III). The initial data of the third 2D RHD Riemann 


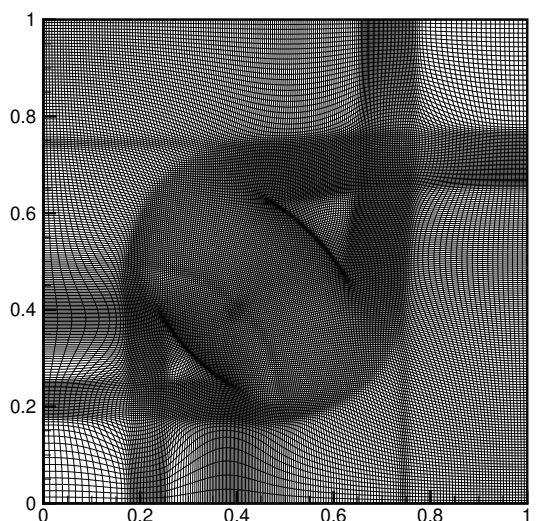

(a) MM-05 with $N=200$

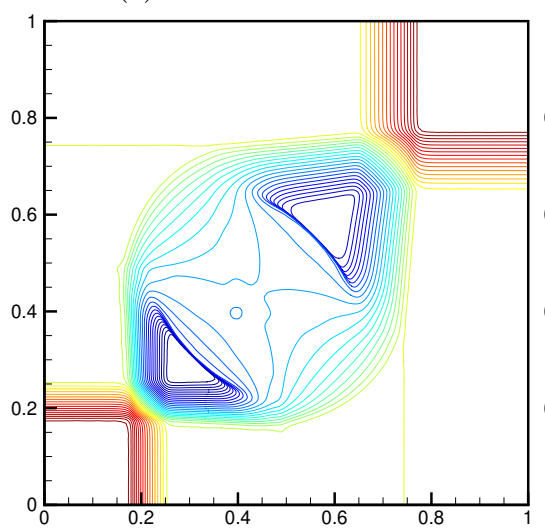

(d) MM-05 with $N=150(35 \mathrm{~s})$

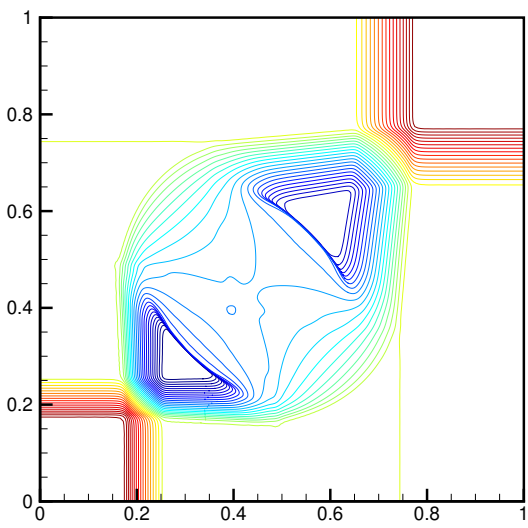

(b) MM-05 with $N=200(1 \mathrm{~m} 25 \mathrm{~s})$

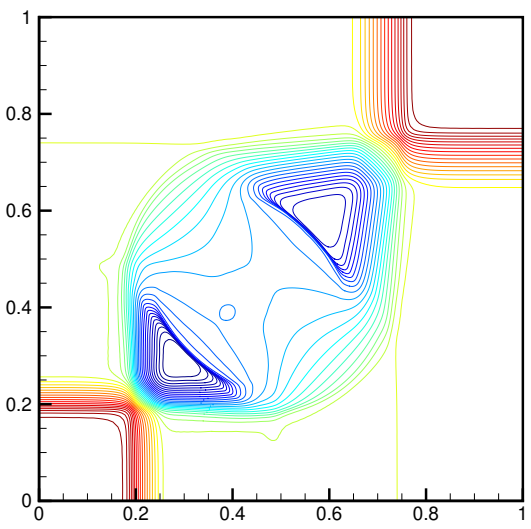

(e) MM-02 with $N=200(38 \mathrm{~s})$

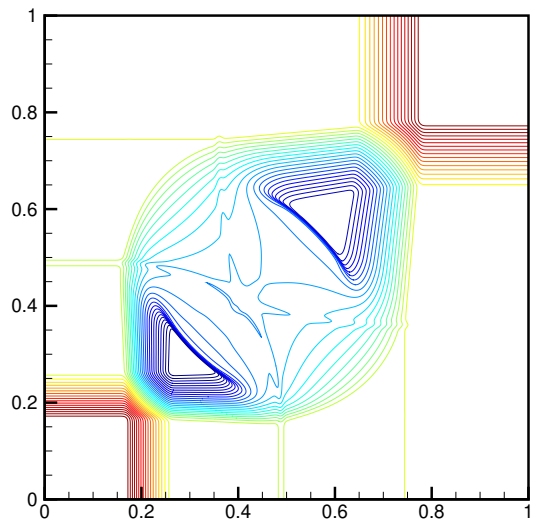

(c) UM-05 with $N=500(4 \mathrm{~m} 38 \mathrm{~s})$

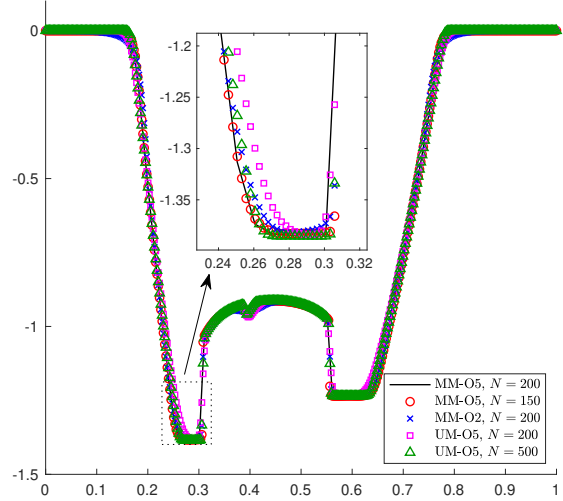

(f) $\ln \rho$ along $x_{2}=x_{1}$

Figure 6.6: Example 6.3. Adaptive mesh of MM-05 with $N=200,40$ equally spaced contour lines of $\ln \rho$, and the cut lines of $\ln \rho$ along $x_{2}=x_{1}$. CPU times are listed in parentheses.

problem are

$$
\left(\rho, v_{1}, v_{2}, p\right)= \begin{cases}(0.035145216124503,0,0,0.162931056509027), & x_{1}>0.5, x_{2}>0.5 \\ (0.1,0.7,0,1), & x_{1}<0.5, x_{2}>0.5 \\ (0.5,0,0,1), & x_{1}<0.5, x_{2}<0.5 \\ (0.1,0,0.7,1), & x_{1}>0.5, x_{2}<0.5\end{cases}
$$

where the left and bottom discontinuities are two contact discontinuities and the top and right are two shock waves.

The monitor function is the same as above. The adaptive mesh of MM-05 with $N=200$, the contours of the density logarithms $\ln \rho$ with 40 equally spaced lines, and $\ln \rho$ cut along $x_{2}=x_{1}$ at $t=0.4$ are shown in Figure 6.7. Similar to the last two examples, from Figure 6.7(d) and 6.7(e), one can see that MM-05 gives better results than MM-02 when using comparable CPU time, especially around the central "mushroom cloud", which forms after the interaction of the initial 
discontinuities. The solution obtained by MM-05 with $N=200$ is much better than UM-05 with $N=200$, see Figure 6.7(f), and agrees well with that of UM-05 with $N=600$, while the adaptive moving mesh scheme only takes $13.7 \%$ CPU time, verifying the high efficiency of our high-order accurate ES adaptive moving mesh schemes.

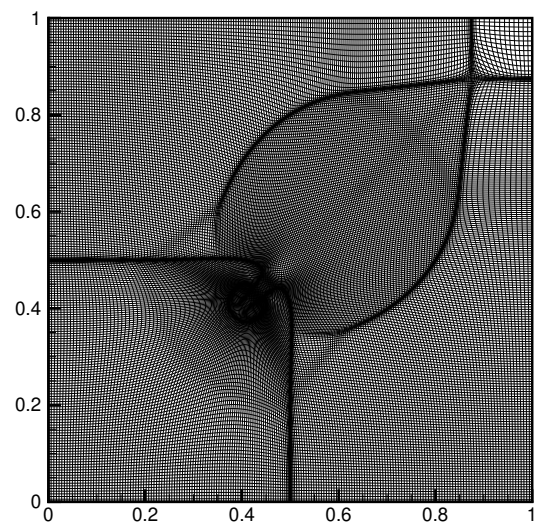

(a) MM-05 with $N=200$

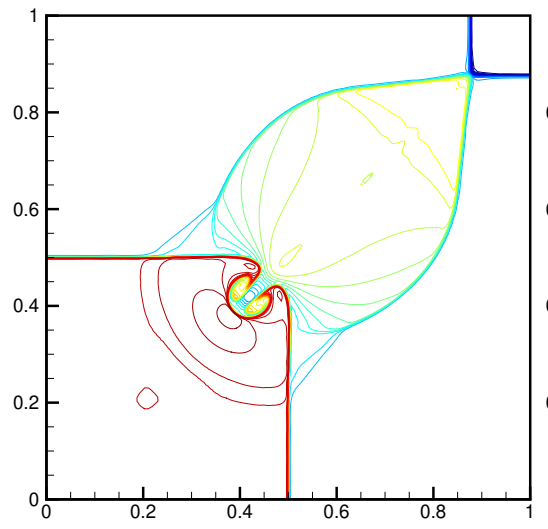

(d) MM-05 with $N=150(32 \mathrm{~s})$

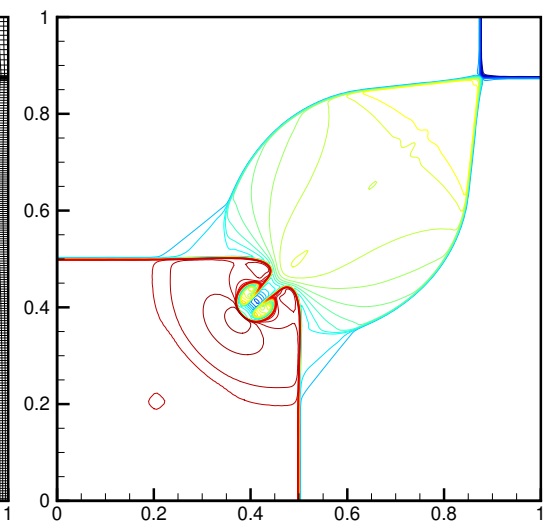

(b) MM-05 with $N=200(1 \mathrm{~m} 16 \mathrm{~s})$

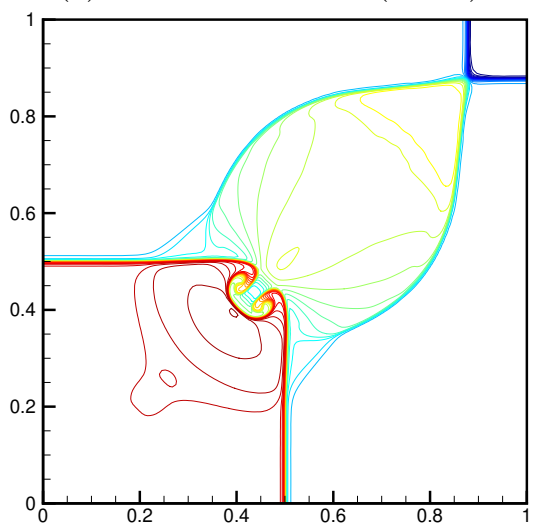

(e) MM-02 with $N=200(39 \mathrm{~s})$

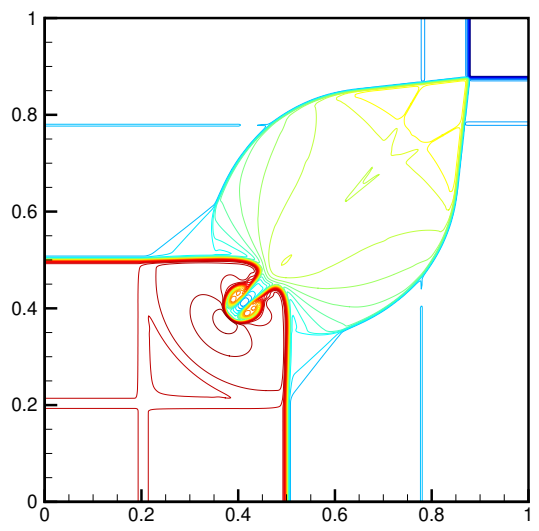

(c) UM-05 with $N=600(9 \mathrm{~m} 16 \mathrm{~s})$

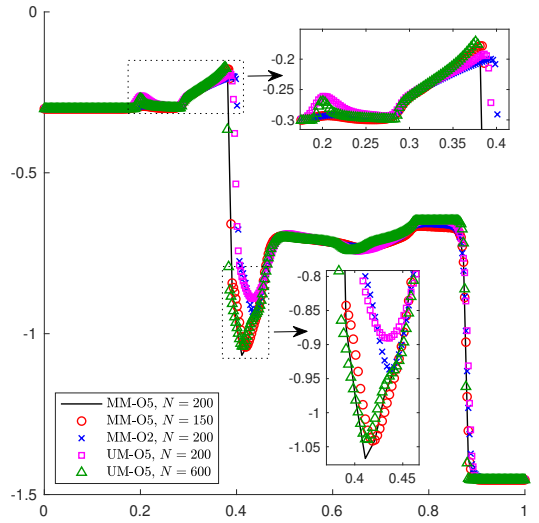

(f) $\ln \rho$ along $x_{2}=x_{1}$

Figure 6.7: Example 6.4. Adaptive mesh of MM-05 with $N=200,40$ equally spaced contour lines of $\ln \rho$, and cut lines of $\ln \rho$ along $x_{2}=x_{1}$. CPU times are listed in parentheses.

Example 6.5 (2D RMHD blast problem). It is a benchmark test problem for the RMHD, and the initial setup in $[3,16,47]$ is adopted. The physical domain is $[-6,6]^{2}$ with outflow boundary conditions, and divided into three parts at initial time. The inner part is the explosion zone with a radius of 0.8 , and $\rho=0.01, p=1$; and the outer part is the ambient medium with the radius larger than 1 , and $\rho=10^{-4}, p=5 \times 10^{-4}$; while the intermediate part is a linear taper applied to the density and the pressure from the radius 0.8 to 1 . The magnetic field is only initialized in the $x_{1}$-direction as $B_{1}=0.1$ and the adiabatic index $\Gamma=4 / 3$. This problem is solved by using the fifth-order ES adaptive moving mesh scheme with $N \times N$ meshes until $t=4$.

The monitor is the same as that in the last example except for $\alpha=800$. Figure 6.8 shows 
the adaptive mesh and 40 equally spaced contour lines obtained by using MM-05 with $150 \times 150$ mesh at $t=4$. One can see that the mesh points adaptively concentrate near the large gradient of $\ln \rho$ due to the choice of the monitor function, and increase the resolution of the shock waves. To compare the results of the fifth-order ES schemes on the adaptive moving mesh and the static uniform mesh, the cut lines of $p$ and $W$ are plotted in Figure 6.9. It is seen that the results obtained by using MM-05 with $N=150$ are much better than those of UM-05 with the same grid number, and comparable to those of UM-05 with $N=600$. From Table 6.1 , one can see that MM-05 is more efficient than UM-05, since the former takes only $7.26 \% \mathrm{CPU}$ time of the latter, highlighting the high efficiency of our high-order accurate ES adaptive moving mesh schemes.

Example 6.6 (2D RMHD shock-cloud interaction). It is about a strong shock wave interacts with a high density cloud [32]. The physical domain is $[-0.2,1.2] \times[0,1]$ with the inflow boundary condition specified on the left boundary, and the outflow boundary conditions on the other boundaries. A planar shock wave moves from $x_{1}=0.05$ to the right with the left and right states

$$
(\rho, \boldsymbol{v}, p, \boldsymbol{B})= \begin{cases}(3.86859,0.68,0,0,1.25115,0,0.84981,-0.84981), & x_{1}<0.05 \\ (1,0,0,0,0,0.16106,0.16106,0.05), & \text { otherwise }\end{cases}
$$

The circular cloud of radius 0.15 with a high density $\rho=30$ is centered at $(0.25,0.5)$. This problem is solved by using the fifth-order ES adaptive moving mesh scheme until $t=1.2$.

The monitor is the same as that in the last example. Figure 6.10 shows the $210 \times 150$ adaptive mesh obtain by MM-05, where the mesh points adaptively concentrate near the cloud. To give comparable results presented in [32], the numerical schlieren images generated by using $\phi_{1}=$ $\exp \left(-50|\nabla \ln \rho| /|\nabla \ln \rho|_{\max }\right)$ and $\phi_{2}=\exp \left(-50|\nabla| \boldsymbol{B}|| /\left.|\nabla| \boldsymbol{B}\right|_{\max }\right)$ are presented in Figures 6.116.12. The results obtained by MM-05 with $210 \times 150$ mesh are shown in the upper half parts, while UM-05 with $210 \times 150$ and $560 \times 400$ meshes are respectively shown in the lower half parts of the left and right plots, so that one can compare the results more clearly. Similar to the last example, MM-05 gives the comparable results to UM-05 with a finer mesh, while takes only $10.5 \%$ CPU time, see Table 6.1.

\begin{tabular}{c|c|c}
\hline Scheme & Example 6.5 & Example 6.6 \\
\hline MM-05 & $2 \mathrm{~m} 03 \mathrm{~s}(150 \times 150)$ & $4 \mathrm{~m} 16 \mathrm{~s}(210 \times 150)$ \\
UM-05 & $30 \mathrm{~s}(150 \times 150)$ & $2 \mathrm{~m} 05 \mathrm{~s}(210 \times 150)$ \\
UM-05 & $28 \mathrm{~m} 14 \mathrm{~s}(600 \times 600)$ & $40 \mathrm{~m} 28 \mathrm{~s}(560 \times 400)$ \\
\hline
\end{tabular}

Table 6.1: CPU times of Examples 6.5-6.6 (4 cores are used). 


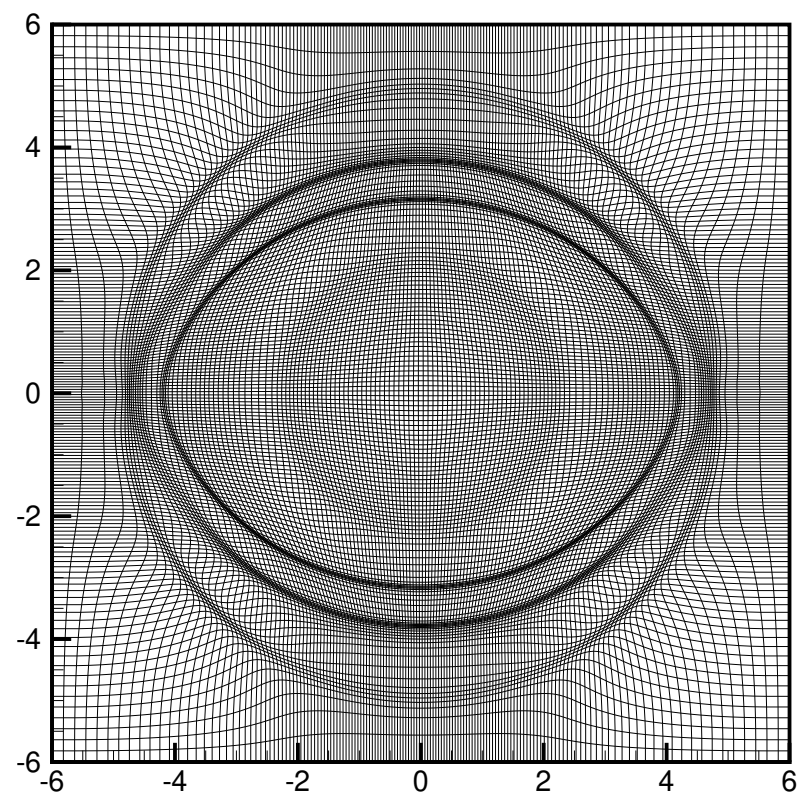

(a) Adaptive mesh

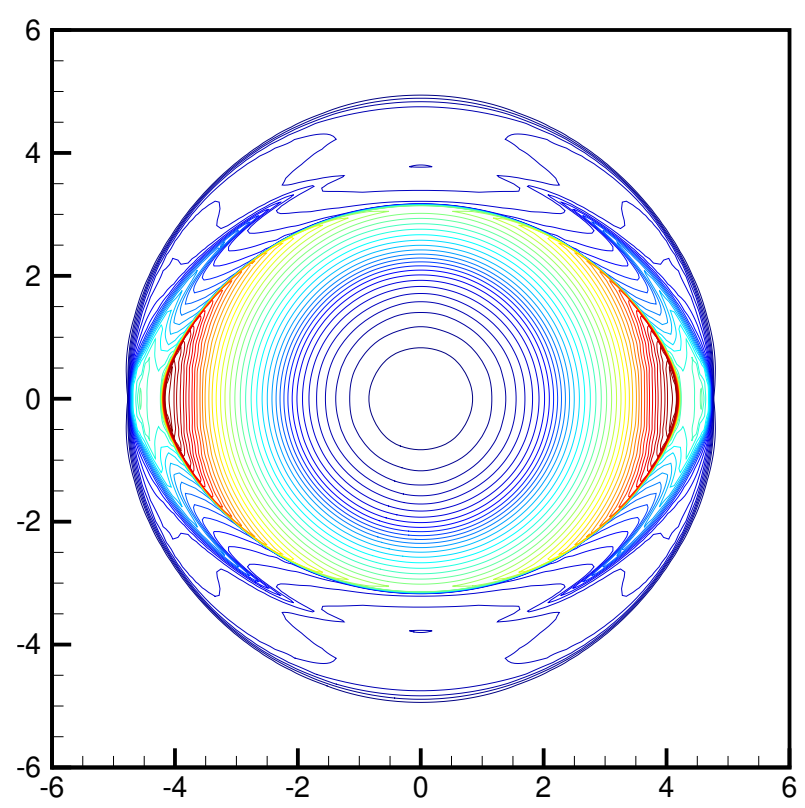

(c) $W$

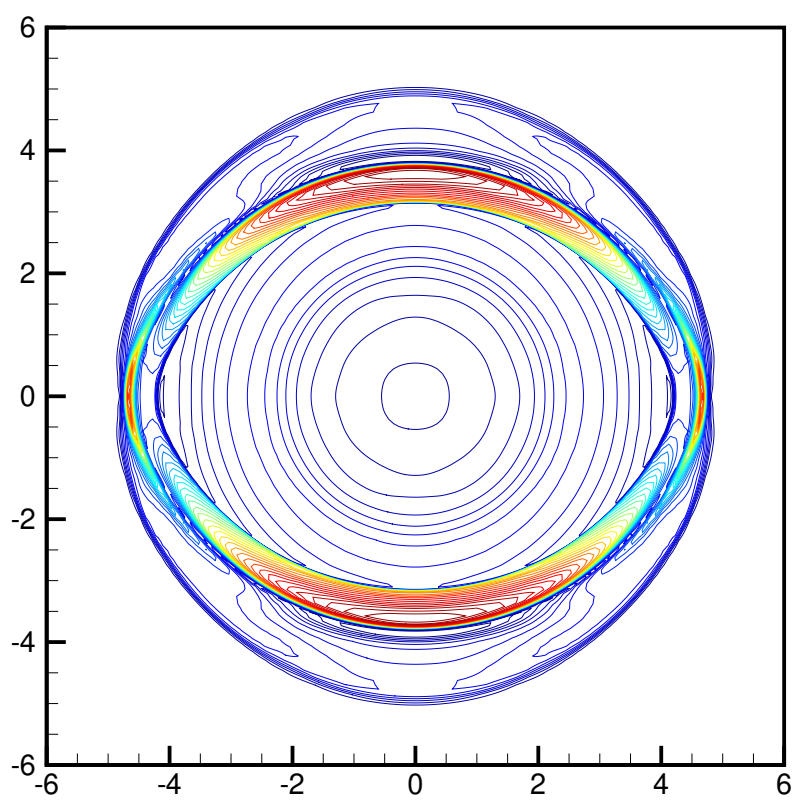

(b) $\rho$

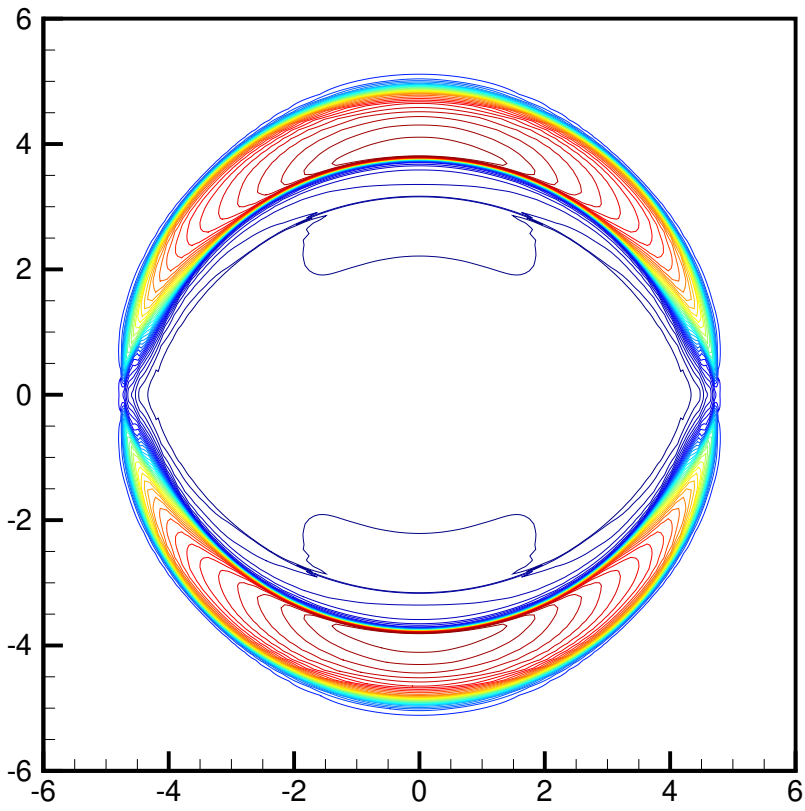

(d) $|\boldsymbol{B}|$

Figure 6.8: Example 6.5. Adaptive mesh and 40 equally spaced contour lines obtained by MM-05 with $150 \times 150$ mesh.

\section{2. $3 D$ tests}

Example 6.7 (3D RMHD isentropic vortex problem). It is given in [18] and used here to verify the accuracy of the 3D EC and ES moving mesh schemes. The analytical solutions at time $t$ and the spatial point $\left(x_{1}, x_{2}, x_{3}\right)$ in the physical domain $[-R, R] \times[-R, R] \times[-5 R, 5 R]$ with $R=5$ and 


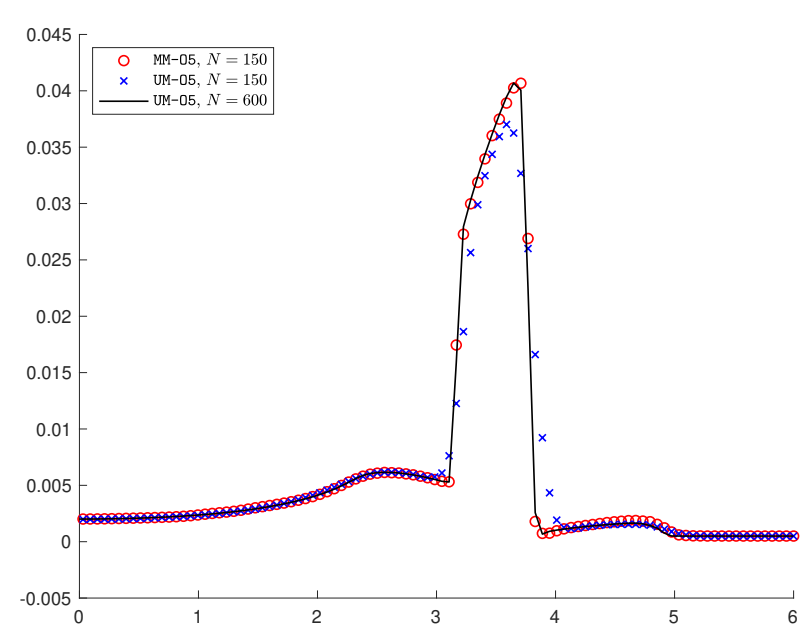

(a) $p$

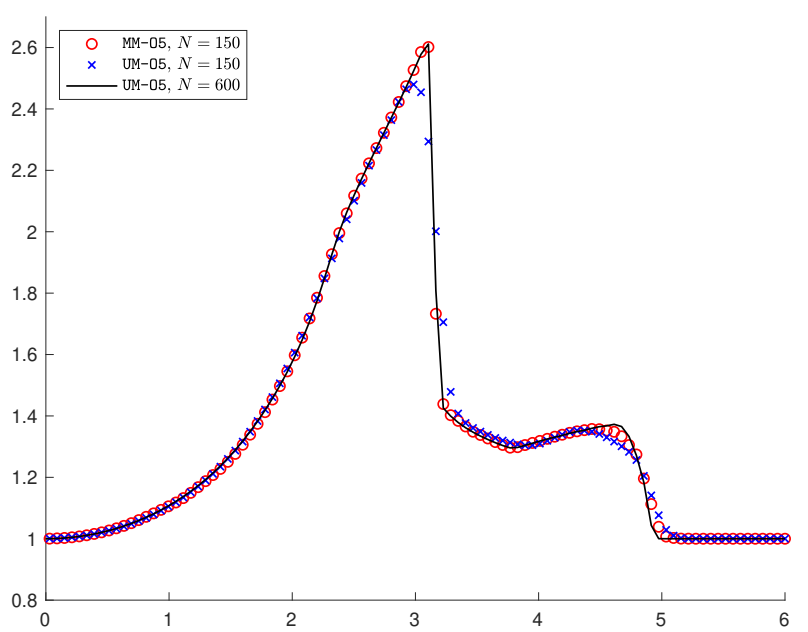

(b) $W$

Figure 6.9: Example 6.5. Cut lines of $p$ and $W$ along $x_{1}=0\left(x_{2} \in[0,6]\right)$.

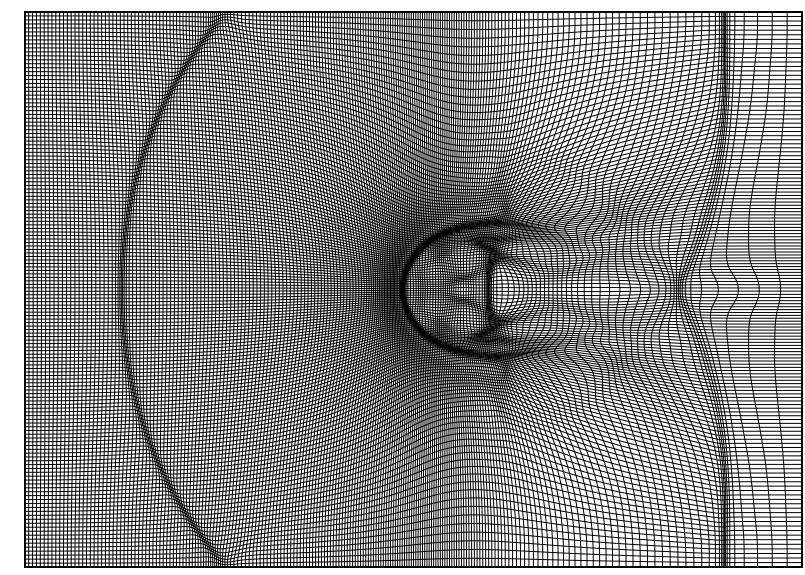

Figure 6.10: Example 6.6. $210 \times 150$ adaptive mesh obtained by MM-05 at $t=1.2$.

the periodic boundary conditions can be given by

$$
\begin{aligned}
\rho & =\left(1-\sigma \exp \left(1-r^{2}\right)\right)^{\frac{1}{\Gamma-1}}, p=\rho^{\Gamma}, \\
\boldsymbol{v} & =\frac{1}{6-3\left(\widetilde{v}_{1}+\widetilde{v}_{2}\right)}\left(4 \widetilde{v}_{1}+\widetilde{v}_{2}-3,4 \widetilde{v}_{2}+\widetilde{v}_{1}-3, \widetilde{v}_{1}+\widetilde{v}_{2}-3\right), \\
\boldsymbol{B} & =\frac{1}{3}\left(5 \widetilde{B}_{1}-\widetilde{B}_{2}, 5 \widetilde{B}_{2}-\widetilde{B}_{1},-\widetilde{B}_{1}-\widetilde{B}_{2}\right),
\end{aligned}
$$



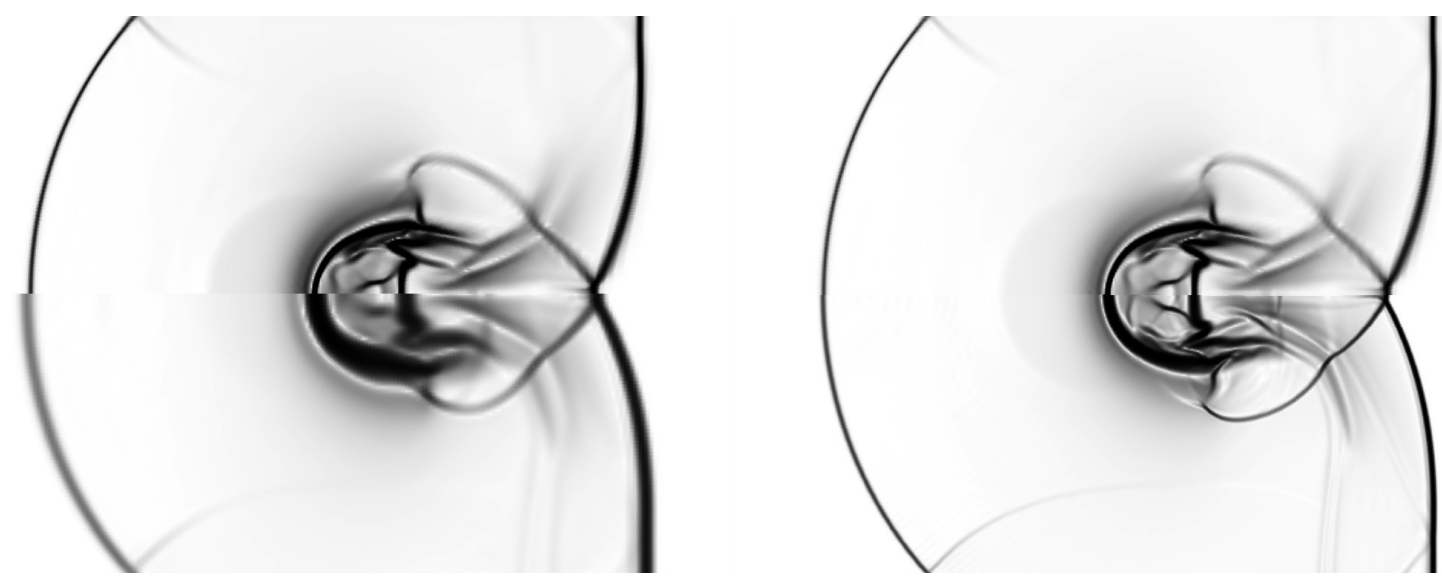

Figure 6.11: Example 6.6. Numerical schlieren images of $\phi_{1}$ at $t=1.2$. Left: MM-05 with $210 \times 150$ mesh (upper half) and UM-05 with $210 \times 150$ mesh (lower half). Right: MM-05 with $210 \times 150$ mesh (upper half) and UM-05 with $560 \times 400$ mesh (lower half).
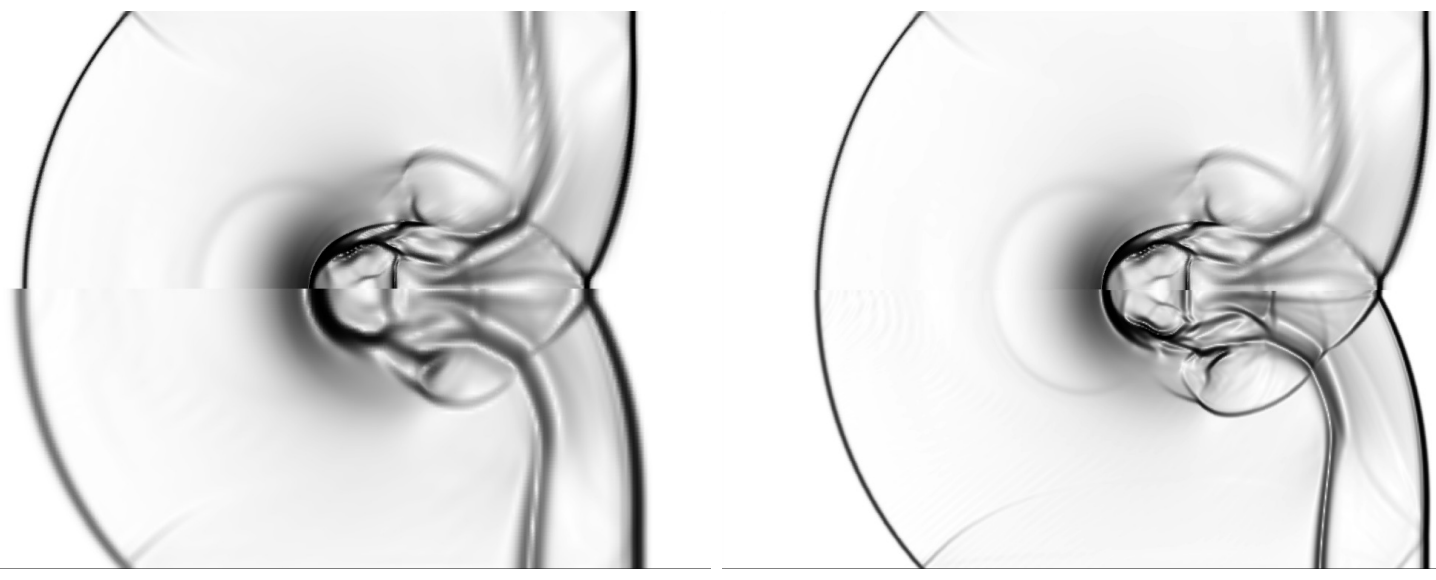

Figure 6.12: Same as Figure 6.11 except for $\phi_{2}$. 
where

$$
\begin{aligned}
& \Gamma=5 / 3, \sigma=0.2, B_{0}=0.05, r=\sqrt{\widetilde{x}_{1}^{2}+\widetilde{x}_{2}^{2}}, \\
& \left(\widetilde{x}_{1}, \widetilde{x}_{2}\right)=\left(40 / 3 k_{1}+10 / 3 k_{2}+\widehat{x}_{1}, 10 / 3 k_{1}+40 / 3 k_{2}+\widehat{x}_{2}\right),\left(\widetilde{x}_{1}, \widetilde{x}_{2}\right) \in \Omega_{0}, k_{1}, k_{2} \in \mathbb{Z}, \\
& \widehat{x}_{k}=x_{k}+\left(x_{1}+x_{2}+x_{3}\right) / 3+t, k=1,2,3, \\
& \left(\widetilde{v}_{1}, \widetilde{v}_{2}\right)=\left(-\widetilde{x}_{2}, \widetilde{x}_{1}\right) f, f=\sqrt{\frac{\kappa \exp \left(1-r^{2}\right)}{\kappa r^{2} \exp \left(1-r^{2}\right)+(\Gamma-1) \rho+\Gamma p}}, \kappa=2 \Gamma \sigma \rho+(\Gamma-1) B_{0}^{2}\left(2-r^{2}\right), \\
& \left(\widetilde{B}_{1}, \widetilde{B}_{2}\right)=B_{0} \exp \left(1-r^{2}\right)\left(-\widetilde{x}_{2}, \widetilde{x}_{1}\right) .
\end{aligned}
$$

The problem is solved until $t=0.1$ with a series of $N \times N \times 5 N$ meshes.

Similar to the $2 \mathrm{D}$ isentropic vortex problem, two mesh movements are used. The first is generated by using the adaptive moving mesh strategy in Section 5 based on the monitor being similar to the $2 \mathrm{D}$ case $(6.3)$, while the second is given by the following expressions

$$
\begin{aligned}
& \left(x_{1}\right)_{i}=\stackrel{\circ}{x}_{1}+0.2 \cos (\pi t / 4) \sin \left(3 \pi \stackrel{\circ}{x}_{2} / R\right) \sin \left(3 \pi \stackrel{\circ}{x}_{3} / 5 R\right), \\
& \left(x_{2}\right)_{i}=\stackrel{\circ}{x}_{2}+0.2 \cos (\pi t / 4) \sin \left(3 \pi \stackrel{\circ}{x}_{3} / 5 R\right) \sin \left(3 \pi \stackrel{\circ}{x}_{1} / R\right), \\
& \left(x_{3}\right)_{i}=\stackrel{\circ}{x}_{3}+0.2 \cos (\pi t / 4) \sin \left(3 \pi \stackrel{\circ}{x}_{1} / R\right) \sin \left(3 \pi \stackrel{\circ}{x}_{2} / R\right), \\
& \stackrel{\circ}{x}_{1}=2 i_{1} R /(N-1), \stackrel{\circ}{x}_{2}=2 i_{2} R /(N-1), i_{1}, i_{2}=0,1, \cdots, N-1, \\
& \stackrel{\circ}{x}_{3}=10 i_{3} R /(5 N-1), i_{3}=0,1, \cdots, 5 N-1 .
\end{aligned}
$$

Figure 6.13 plots the errors and convergence orders in $\rho$, from which one can see that MM-05 with the adaptive moving mesh gets fifth-order, while MM-06 with the moving mesh (6.4) achieves sixth-order accuracy. Figure 6.14 presents the time evolution of the discrete total entropy $\sum_{\boldsymbol{i}} J_{\boldsymbol{i}} \eta\left(\boldsymbol{U}_{\boldsymbol{i}}\right) / 5 / N^{3}$ obtained by MM-06 and MM-05 with $N=160$, verifying the EC and ES property of our schemes.
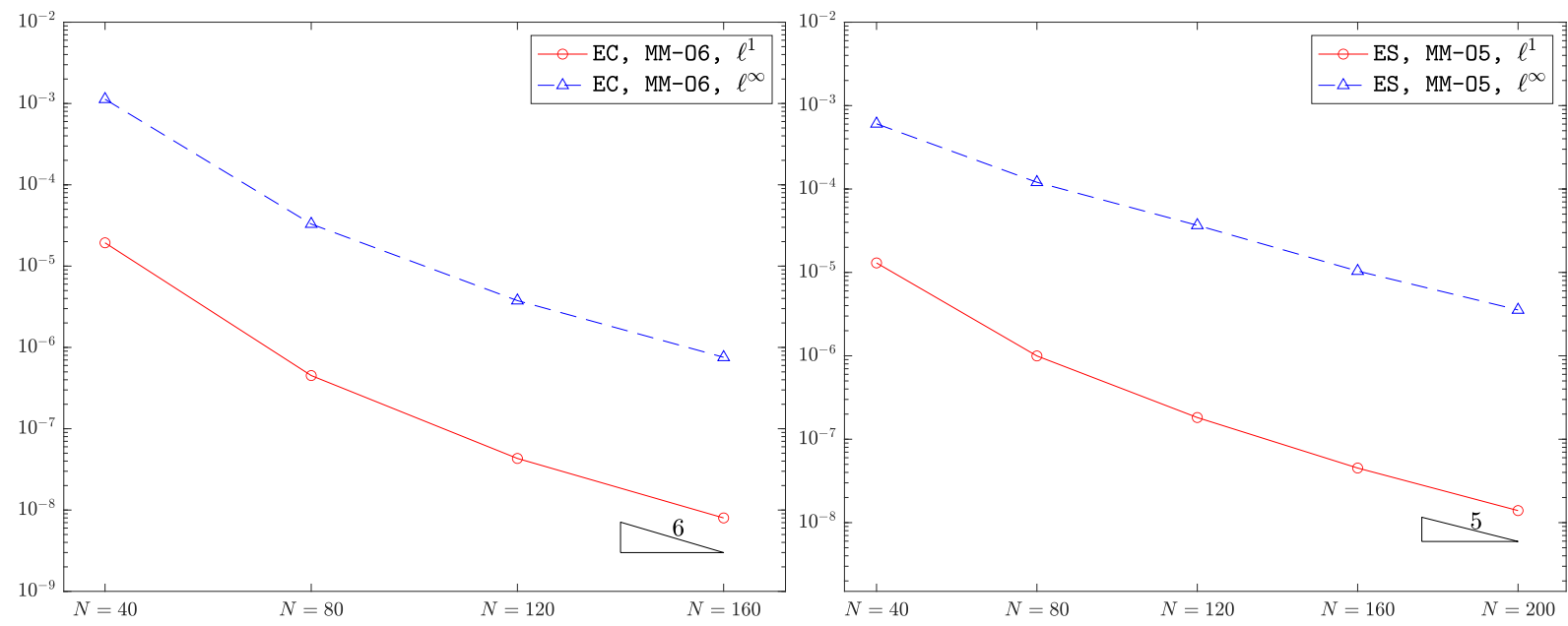

Figure 6.13: Example 6.7. Errors and convergence orders in $\rho$ at $t=0.1$. 


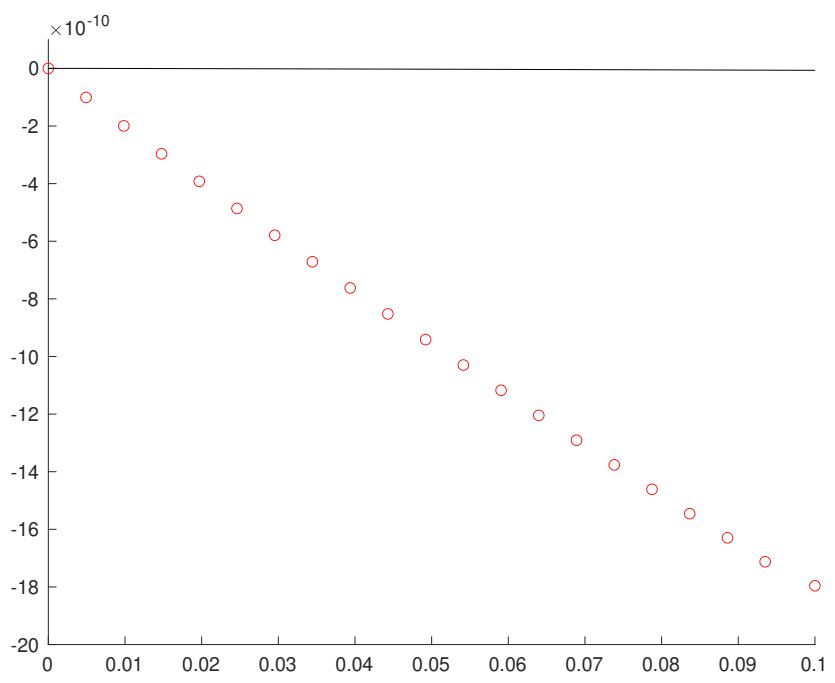

Figure 6.14: Example 6.7. Discrete total entropy obtained by EC and ES schemes with $N=160$.

Example 6.8 (3D RHD spherical symmetric Riemann problem). This problem has a reference solution so that it is suitable to serve as the first example to verify our 3D high-order accurate ES adaptive moving mesh schemes. The reference solution is obtained by using a second-order TVD scheme to solve the RHD equations in the $1 \mathrm{D}$ spherical coordinates. The initial data are

$$
(\rho, \boldsymbol{v}, p)= \begin{cases}(10,0,0,0,40 / 3), & r=\sqrt{x_{1}^{2}+x_{2}^{2}+x_{3}^{2}}<0.5 \\ \left(1,0,0,0,10^{-2}\right), & \text { otherwise }\end{cases}
$$

and $N \times N \times N$ meshes are used.

The monitor function is chosen as (5.3) with $\alpha=800$ and $\sigma=\ln \rho$. Figure 6.15 gives the $100 \times 100 \times 100$ adaptive mesh obtained by MM-05, and the comparison of $\rho$ along the volume diagonal connecting $(0,0,0)$ and $(1,1,1)$ at $t=0.4$. Table 6.2 lists the CPU times of different cases. It is obvious that all the schemes give correct solutions, and the mesh points adaptively concentrate near where the large gradient in $\ln \rho$ occurs, increasing the discontinuity resolution. MM-05 gives better results than MM-02 near the head and tail of the rarefaction wave, indicating that the present high-order accurate scheme outperforms the second-order scheme. The results of MM-05 with $N=100$ and UM-05 with $N=200$ are comparable, while the former costs $13.8 \% \mathrm{CPU}$ time, verifying the efficiency of our high-order accurate ES adaptive moving mesh scheme.

Example 6.9 (3D RHD shock-bubble interaction). This example considers a moving planar shock wave interacts with a light bubble within the physical domain $[0,325] \times[-45,45] \times[-45,45]$, which 


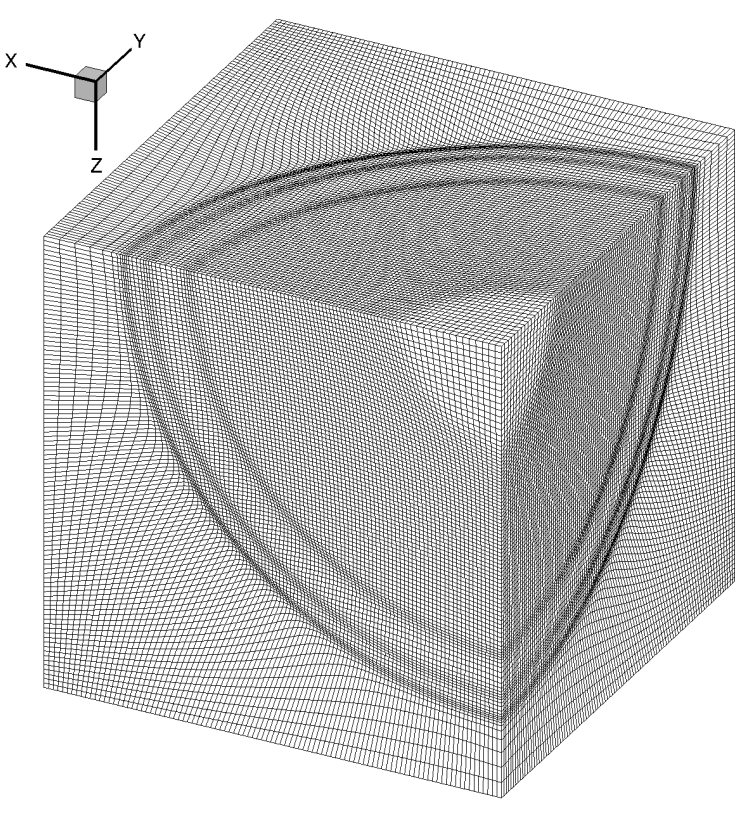

(a) Adaptive mesh of MM-05 with $N=100$

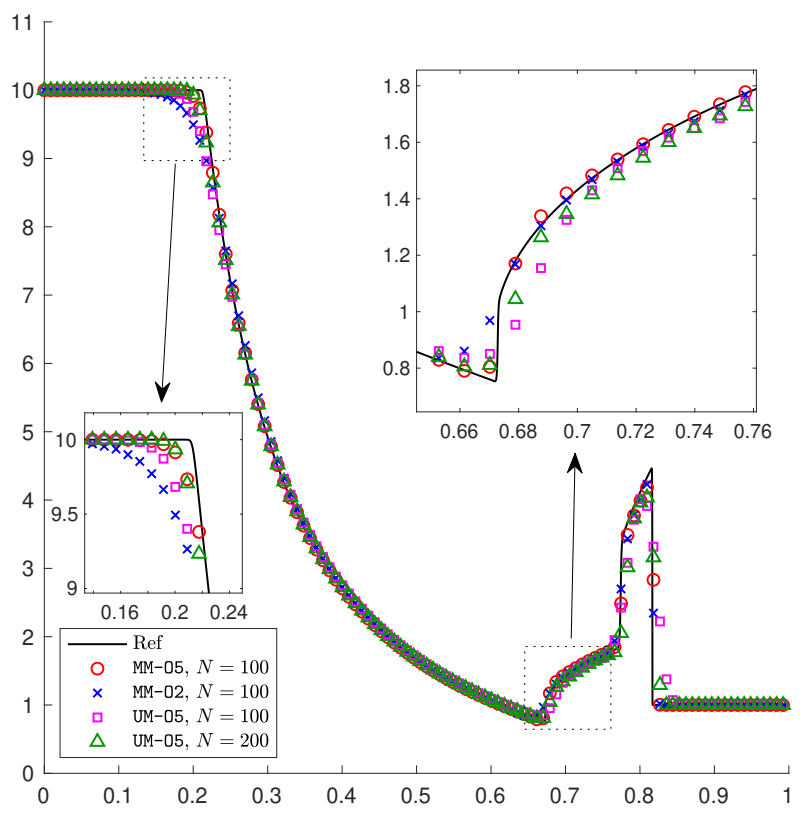

(b) $\rho$ along the line connecting $(0,0,0)$ and $(1,1,1)$

Figure 6.15: Example 6.8. Adaptive mesh and cut lines of $\rho$ at $t=0.4$.

\begin{tabular}{c|c|c|c}
\hline Scheme & Example 6.8 & Example 6.9 & Example 6.10 \\
\hline MM-05 & $5 \mathrm{~m} 40 \mathrm{~s}(100 \times 100 \times 100)$ & $2 \mathrm{~h} 14 \mathrm{~m} 44 \mathrm{~s}(325 \times 90 \times 90)$ & $3 \mathrm{~h} 9 \mathrm{~m} 57 \mathrm{~s}(210 \times 150 \times 150)$ \\
MM-02 & $2 \mathrm{~m} 51 \mathrm{~s}(100 \times 100 \times 100)$ & $1 \mathrm{~h} 10 \mathrm{~m} 29 \mathrm{~s}(325 \times 90 \times 90)$ & - \\
UM-05 & $3 \mathrm{~m} 08 \mathrm{~s}(100 \times 100 \times 100)$ & $51 \mathrm{~m} 18 \mathrm{~s}(325 \times 90 \times 90)$ & $2 \mathrm{~h} 8 \mathrm{~m} 44 \mathrm{~s}(210 \times 150 \times 150)$ \\
UM-05 & $41 \mathrm{~m} 08 \mathrm{~s}(200 \times 200 \times 200)$ & $12 \mathrm{~h} 34 \mathrm{~m} 43 \mathrm{~s}(650 \times 180 \times 180)$ & $34 \mathrm{~h} 46 \mathrm{~m} 49 \mathrm{~s}(420 \times 300 \times 300)$ \\
\hline
\end{tabular}

Table 6.2: CPU times of Examples 6.8-6.10 (32 cores are used).

is extended from the $2 \mathrm{D}$ case [31], and also used in [21]. The initial pre- and post-shock states are

$$
(\rho, \boldsymbol{v}, p)= \begin{cases}(1,0,0,0,0.05), & x_{1}<265 \\ (1.865225080631180,-0.196781107378299,0,0,0.15), & x_{1}>265\end{cases}
$$

and the state in the bubble is

$$
(\rho, \boldsymbol{v}, p)=(0.1358,0,0,0,0.05), \quad \sqrt{\left(x_{1}-215\right)^{2}+x_{2}^{2}+x_{3}^{2}} \leqslant 25 .
$$

The output times are $t=90,180,270,360,450$.

The monitor is the same as that in the last example. Figure 6.16 presents the iso-surfaces of $\rho=0.7$, the close-up of the adaptive mesh and two surface meshes near the bubble at $t=450$. One can see that the mesh points concentrate near the shock wave and the bubble according to the choice of the monitor function, which helps to obtain the sharp interfaces. Figure 6.17 gives the adaptive 
meshes and numerical schlieren images generated by $\phi=\exp \left(-10|\nabla \rho| /|\nabla \rho|_{\max }\right)$ on the slice $x_{2}=0$ at $t=90,180,270,360,450$ (from top to bottom). The results obtained by MM-05 with $325 \times 90 \times 90$ meshes are shown in the upper half parts in each row, while the adaptive meshes and numerical schlieren images obtained by MM-02 with $325 \times 90 \times 90$ meshes are shown in the left and middle lower half parts in each row, respectively, and those obtained by UM-05 with $650 \times 180 \times 180$ meshes are shown in the right lower half parts. Those plots clearly show the dynamics of the interaction between the shock wave and the bubble, and our high-order accurate ES adaptive moving mesh schemes well capture the sharp interfaces of the bubble at different output times. One can see that as time increases, the fifth-order scheme gives sharper interfaces than the second-order scheme, since the high-order accurate scheme has lower dissipation. From the CPU times listed in Table 6.2, MM-05 is more efficient than UM-05, because it takes only $17.8 \% \mathrm{CPU}$ time to give comparable results.

Example 6.10 (3D RMHD shock-cloud interaction). It is a 3D extension of Example 6.6. The physical domain is $[-0.2,1.2] \times[0,1] \times[0,1]$, and the circular cloud is modified as a spherical cloud of radius 0.15 centered at $(0.25,0.5,0.5)$ with invariant density. The initial data of the pre- and post-shock remain unchanged. This problem is solved by using the fifth-order ES adaptive moving mesh scheme until $t=1.2$.

The monitor is the same as the last example. The iso-surfaces of $\rho=1.52$, the close-up of the adaptive mesh and two surface meshes near the bubble at $t=1.2$ are given in Figure 6.18. The mesh points adaptively concentrate near the complicated structures formed after the interaction of the shock wave and the cloud, improving the nearby resolution. Figures 6.19-6.20 show the numerical schlieren images of $\phi_{1}$ and $\phi_{2}$ defined in Example 6.6 on the slice $x_{2}=0$. The results obtained by MM-05 with $210 \times 150 \times 150$ meshes are plotted in the upper half parts, while those obtained by UM-0 5 with $210 \times 150 \times 150$ and $420 \times 300 \times 300$ meshes are shown in the left and right lower half parts, respectively. One can see that MM-05 gives better results than UM-05 with the same grid number, and the former takes only $9.06 \% \mathrm{CPU}$ time to give comparable results when the latter uses finer mesh, which again shows the high efficiency of our high-order accurate ES adaptive moving mesh schemes.

\section{Conclusions}

This paper presented the high-order accurate ES adaptive moving mesh schemes for the 2D and 3D special RHD and RMHD equations. Our schemes were built on the ES finite difference approximation in the curvilinear coordinates, the discrete GCLs, and the adaptive mesh redistri- 

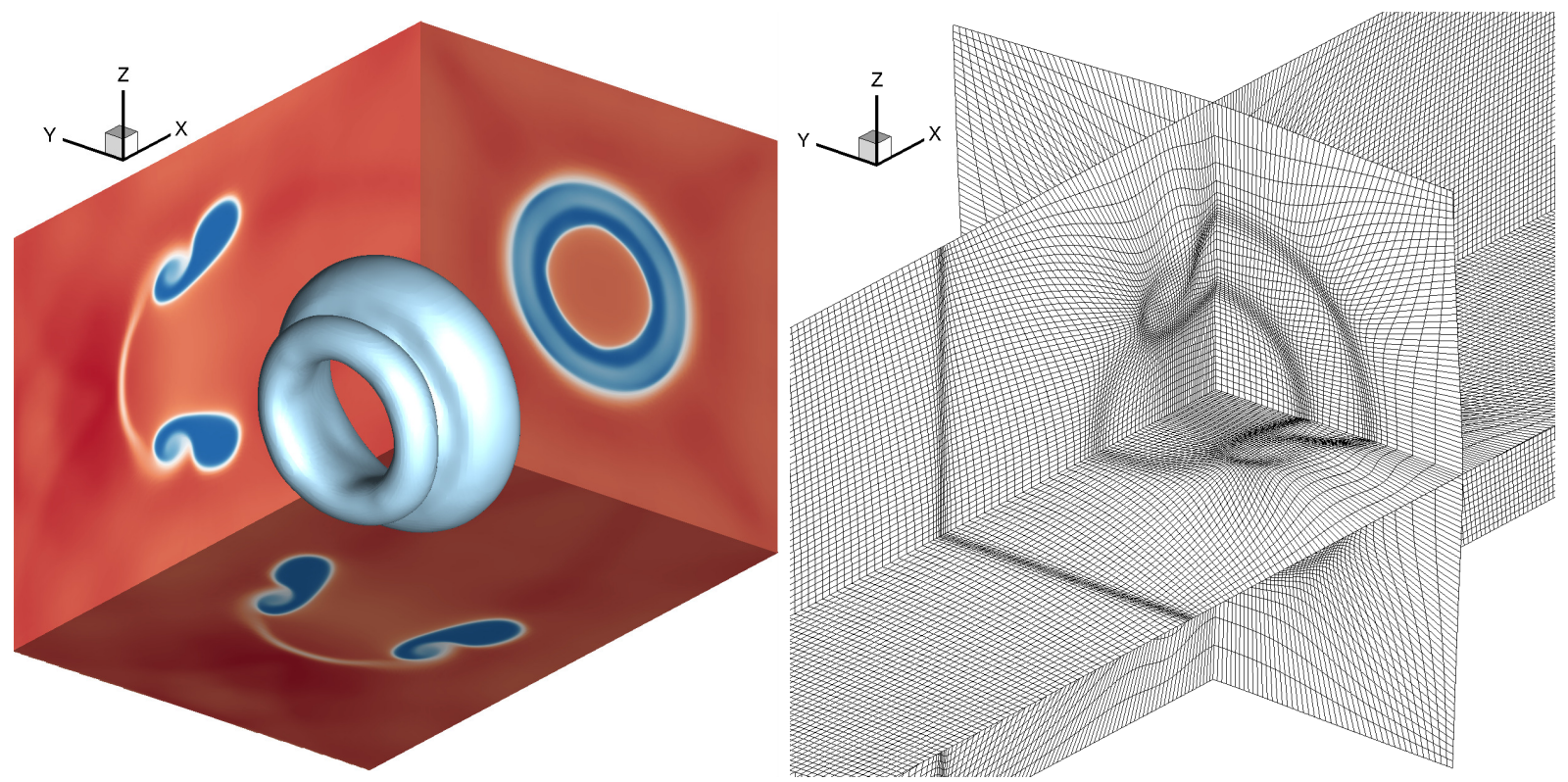

(a) Iso-surface of $\rho=0.7$ and three offset 2D slices taken at $x_{1}=125, x_{2}=0, x_{3}=0$

(b) Adaptive meshes on three surfaces of $i_{1}=125, i_{2}=$

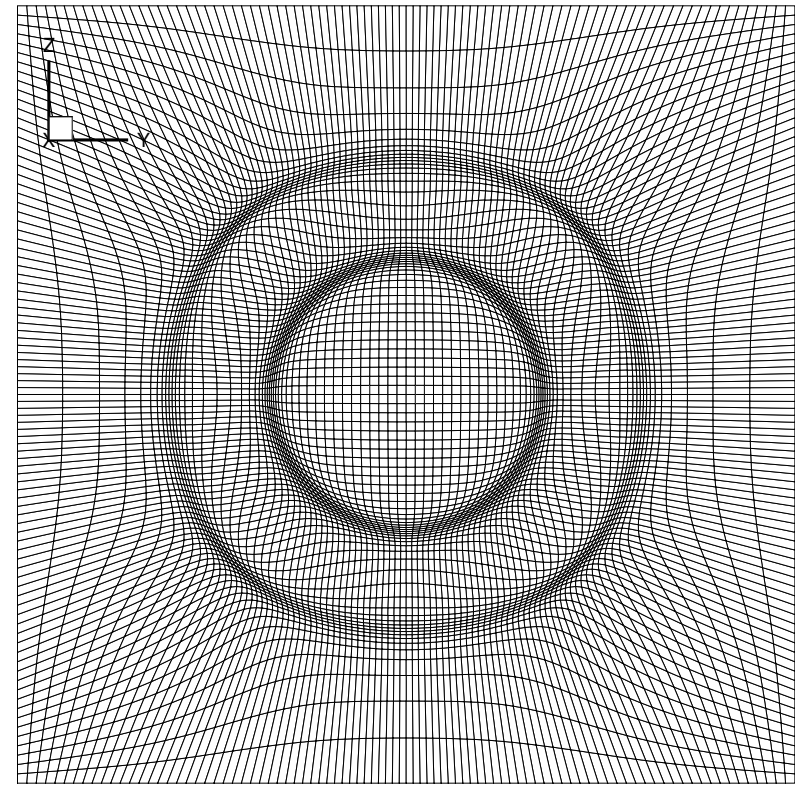

(c) Close-up of adaptive mesh on surface of $i_{1}=125$
$45, i_{3}=45$

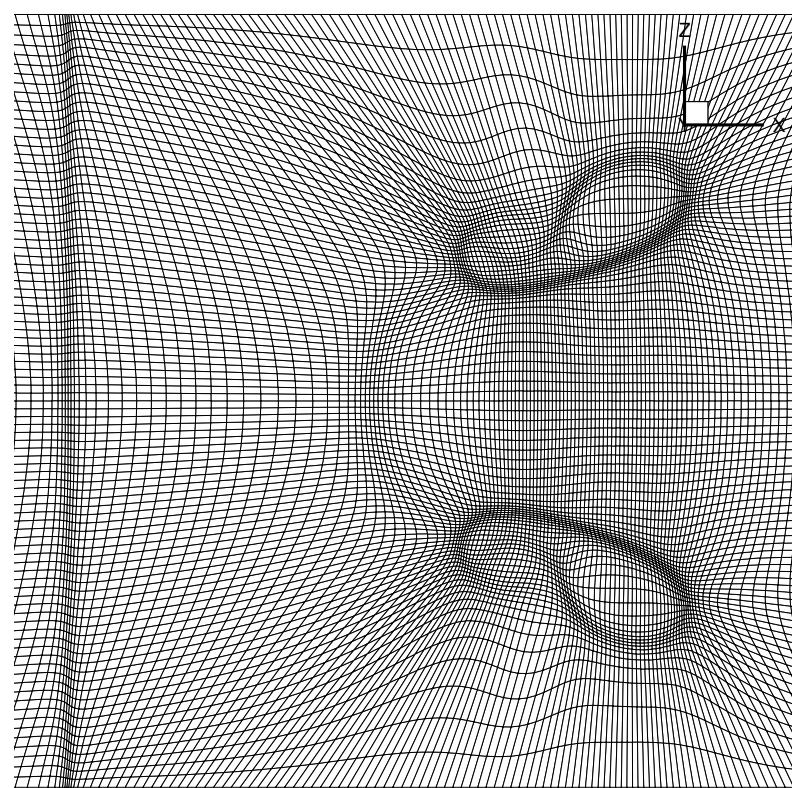

(d) Close-up of adaptive mesh on surface of $i_{2}=45$

Figure 6.16: Example 6.9. Adaptive meshes and $\rho$ at $t=450$.

bution built on the minimization of the mesh adaption functional, and consisted of the following main parts.

1. The two-point EC flux $\widetilde{\mathcal{F}_{k}}$ for the modified RMHD equations (involving the RHD equations) in the curvilinear coordinates for the given entropy pair was first derived, see (3.2), and then the high-order EC flux $\left(\widetilde{\mathcal{F}}_{k}\right)_{i, k, \pm \frac{1}{2}}^{2 p \text { th }}$ was proposed by using some linear combinations of the two- 

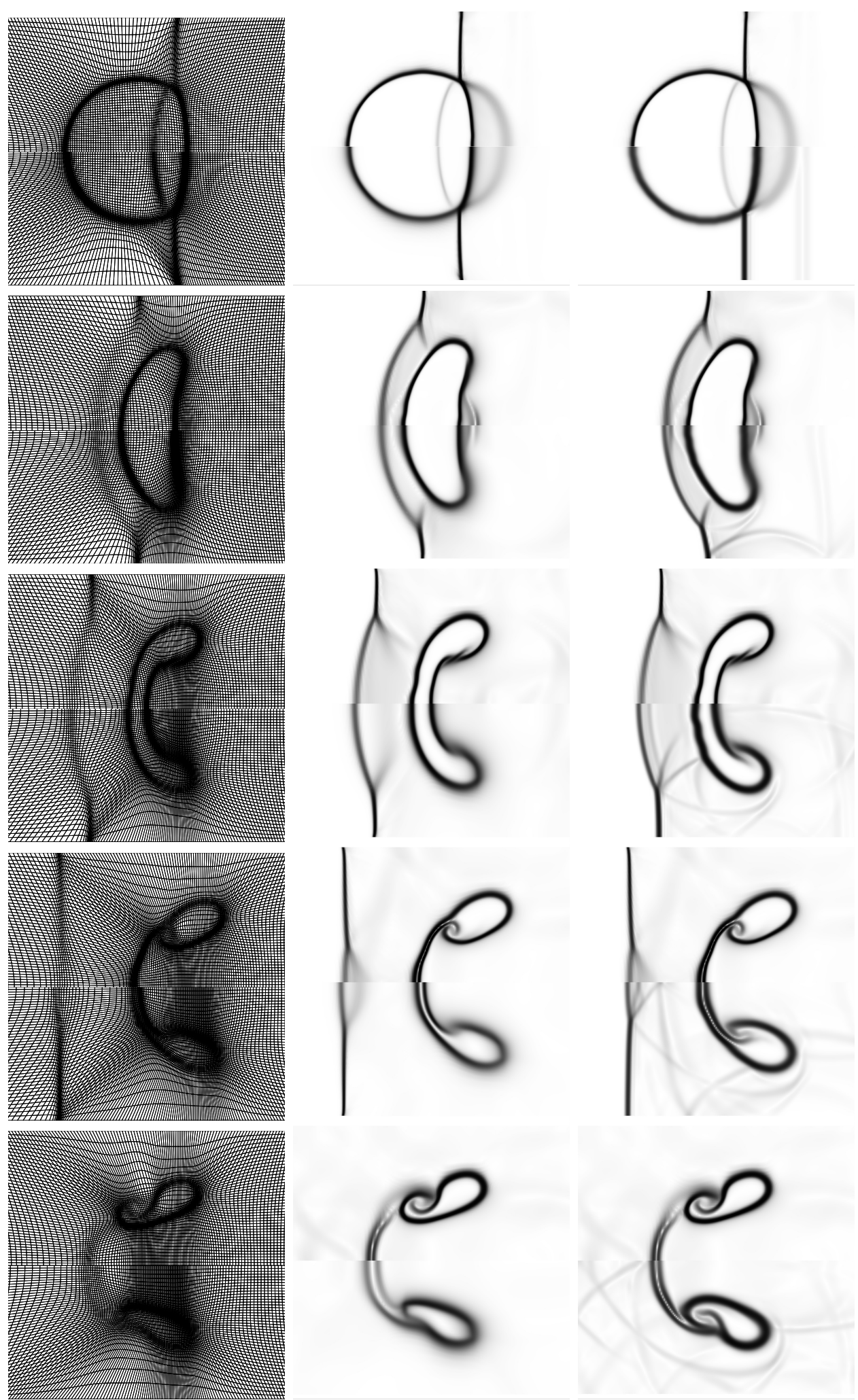

Figure 6.17: Example 6.9. From top to bottom: $t=90,180,270,360,450$. Left: adaptive meshes on surface of $i_{2}=45$ obtained with MM-05 (upper half) and MM-02 (lower half) with $325 \times 90 \times 90$ mesh. Middle: numerical schlieren images of $\phi$ on slice $x_{2}=0$ obtained with MM-05 (upper half) and MM-02 (lower half) with $325 \times 90 \times 90$ mesh. Right: numerical schlieren images of $\phi$ on the slice $x_{2}=0$, obtained with MM-05 (upper half) with $325 \times 90 \times 90$ mesh and UM-05 (lower half) with $650 \times 180 \times 180$ mesh. 41 

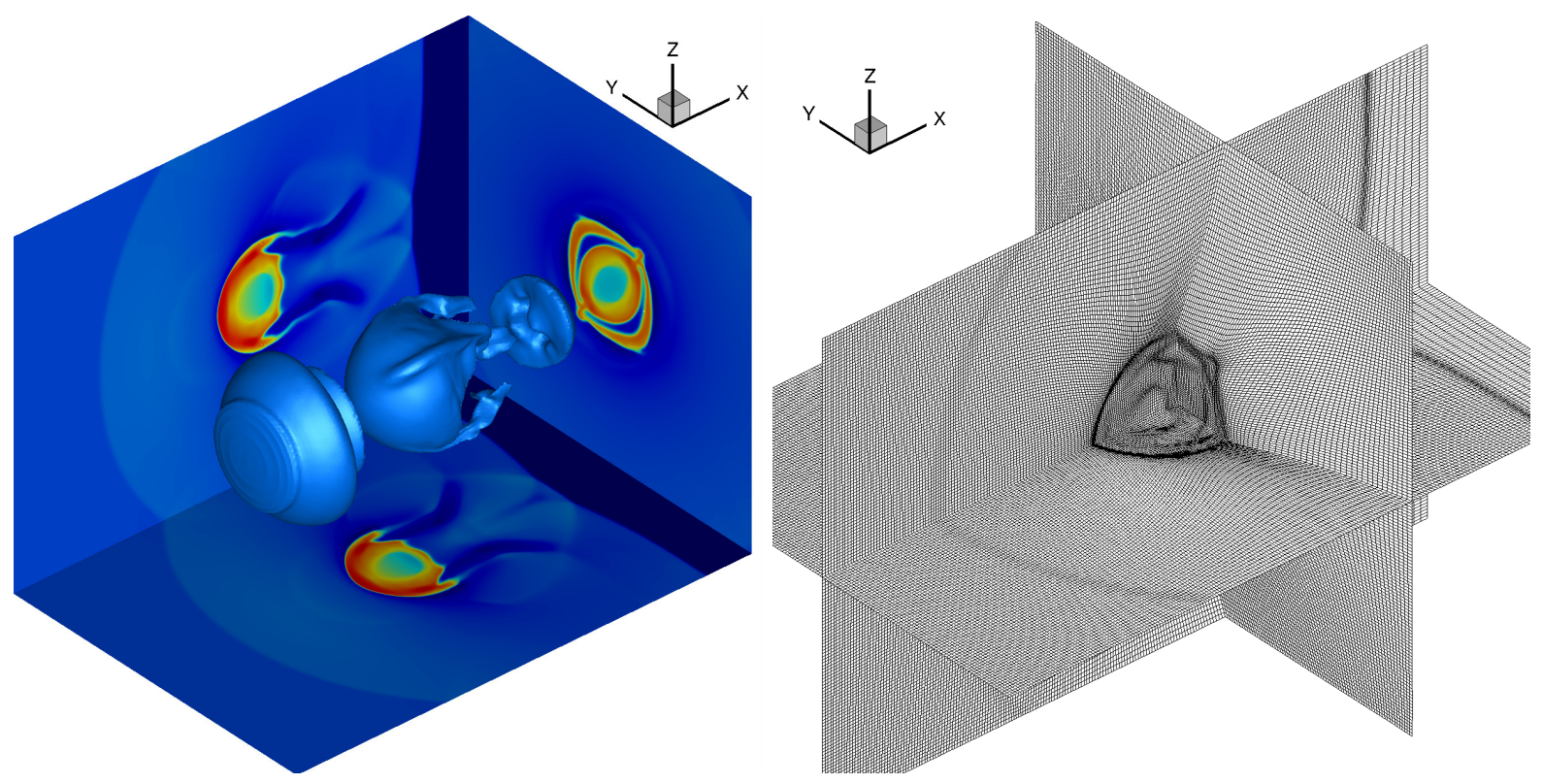

(a) Iso-surface of $\ln \rho=1.52$ and three offset $2 \mathrm{D}$ slices taken

(b) Adaptive meshes on three surfaces of $i_{1}=150, i_{2}=$ at $x_{1}=0.58, x_{2}=0.5, x_{3}=0.5$ $75, i_{3}=75$

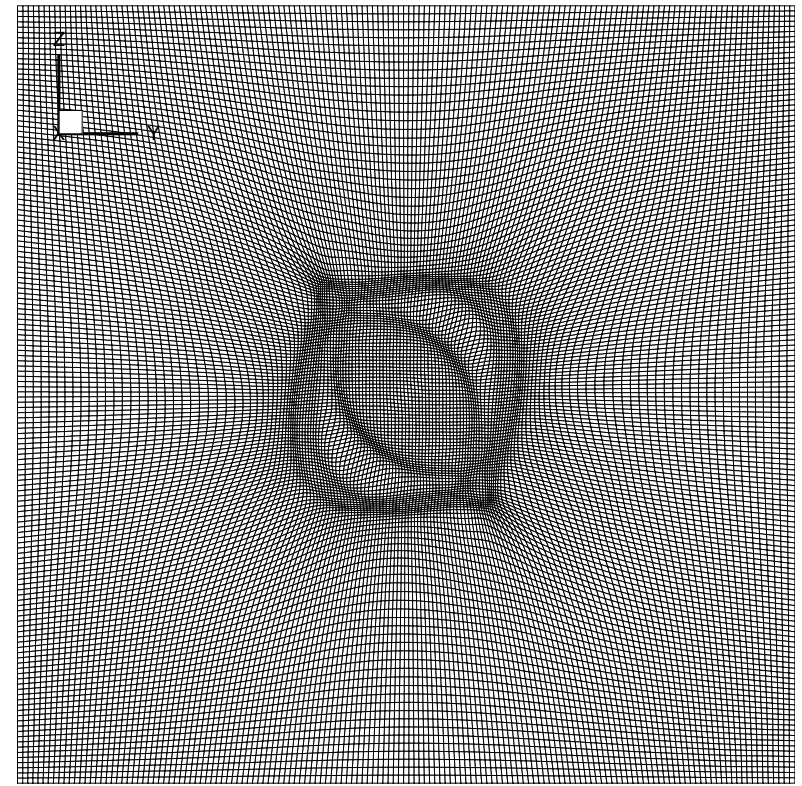

(c) Close-up of adaptive mesh on surface of $i_{1}=150$

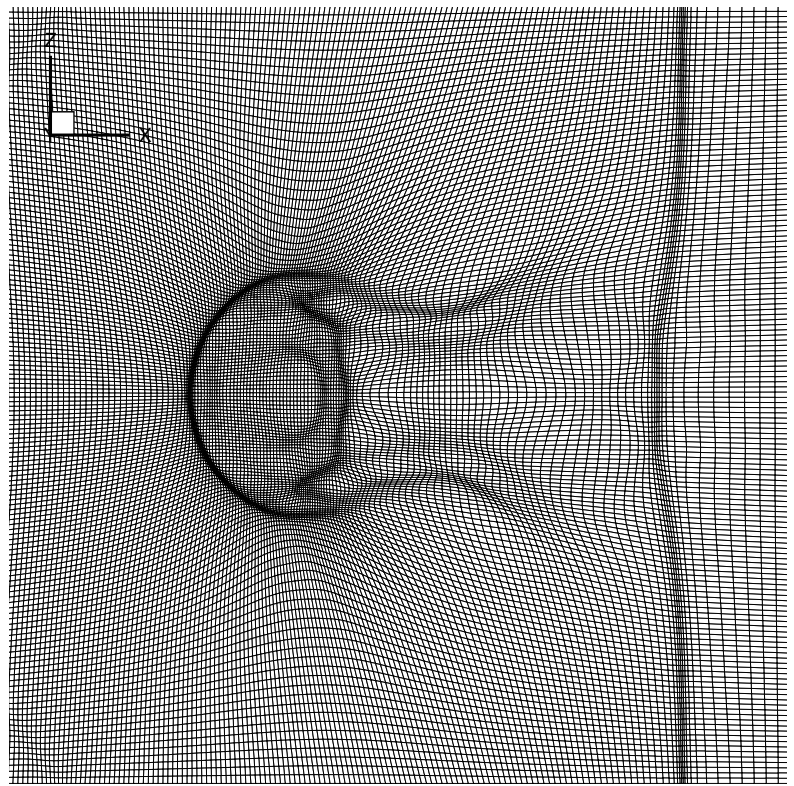

(d) Close-up of adaptive mesh on surface of $i_{2}=75$

Figure 6.18: Example 6.10. Adaptive meshes and $\ln \rho$ at $t=1.2$.

point EC flux $\widetilde{\mathcal{F}}_{k}$, so that the approximation of the flux derivatives in space was $2 p$ th-order accurate, which was an extension of the high-order accurate EC schemes in the Cartesian coordinates [38] to the curvilinear coordinates.

2. The $2 p$ th-order accurate approximations of the spatial derivatives in the source terms and 

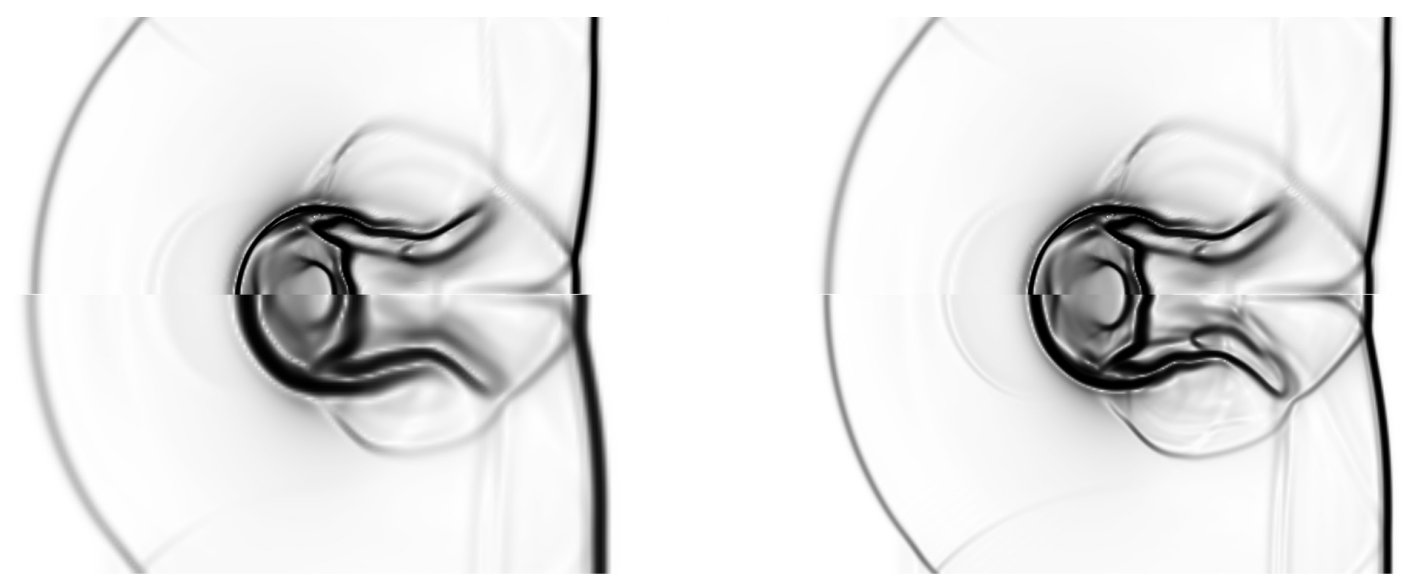

Figure 6.19: Example 6.10. Numerical schlieren images of $\phi_{1}$ at $t=1.2$. Left: MM-05 with $210 \times 150 \times 150$ mesh (upper half) and UM-05 with $210 \times 150 \times 150$ mesh (lower half). Right: MM-05 with $210 \times 150 \times 150$ mesh (upper half) and UM-05 with $420 \times 300 \times 300$ mesh (lower half).
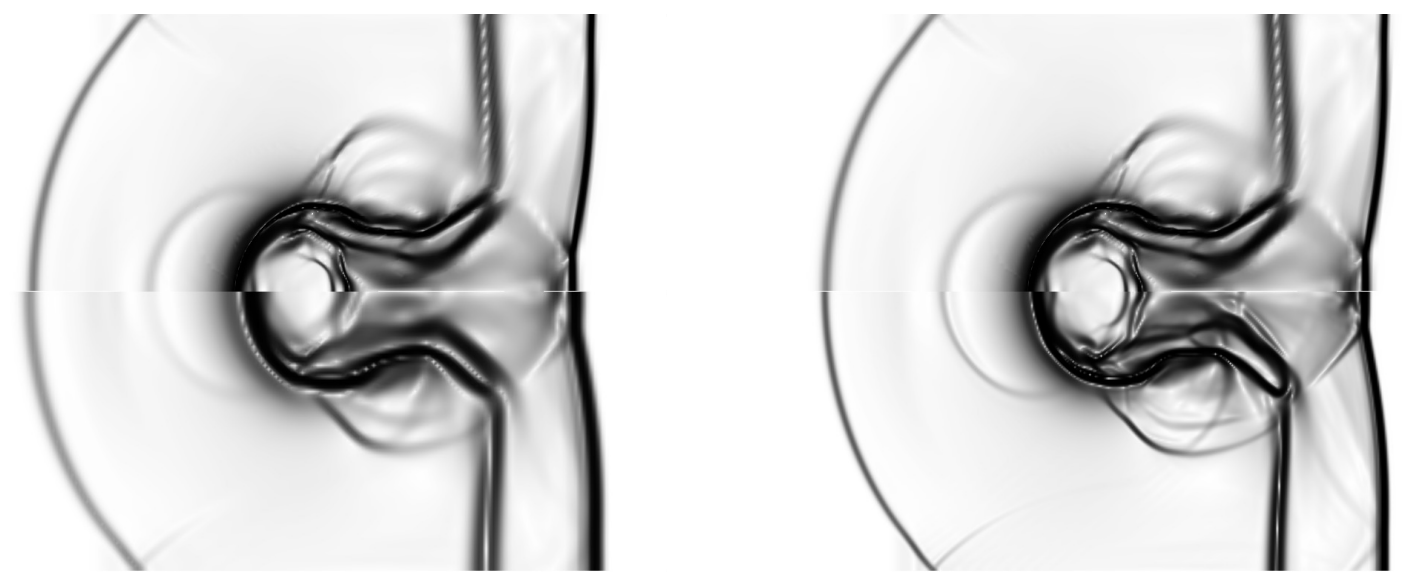

Figure 6.20: Same as Figure 6.19 except for $\phi_{2}$. 
the VCL were given by designing $\left(\widetilde{\mathcal{B}_{k}}\right)_{i, k, \pm \frac{1}{2}}^{2 p t h}$ and $\left(\widetilde{J \frac{\partial \xi_{k}}{\partial t}}\right)_{i, k, \pm \frac{1}{2}}^{2 p \text { th }}$ as the linear combination of corresponding 2nd-order case with the same coefficients as above. The discretization of the latter degenerated to the $2 p$ th-order accurate central difference.

3. The spatial metrics $\left(\widetilde{J \frac{\partial \xi_{k}}{\partial x_{j}}}\right)_{i}$ used in the above two parts were discretized by using the $2 p$ thorder central difference based on the conservative metrics method (CMM) [60], such that the SCLs held in the discrete level.

4. The semi-discrete schemes built on the above three parts, see (3.4)-(3.5), were proved to be $2 p$ th-order accurate in space and EC by mimicking the derivation of the continuous entropy identity in the curvilinear coordinates.

5. Some suitable high-order dissipation term utilizing WENO reconstruction in the scaled entropy variables was added to the EC flux to get the high-order accurate ES schemes satisfying the semi-discrete entropy inequality, in order to avoid the numerical oscillation produced by the EC scheme around the discontinuities.

6. The fully-discrete ES schemes were obtained by integrating the above semi-discrete ES schemes in time by using the third-order accurate explicit strong-stability preserving Runge-Kutta schemes, and proved to be free-stream preserving.

7. The mesh points were adaptively redistributed by solving the Euler-Lagrange equation of the mesh adaption functional on the computational mesh at each time step with the suitably chosen monitor functions.

Several 2D and 3D numerical results showed that the high-order accurate ES adaptive moving mesh schemes effectively captured the localized structures, such as the sharp transitions or discontinuities, and outperformed both their counterparts on the uniform mesh and the 2nd-order ES adaptive moving mesh schemes.

\section{Acknowledgments}

The authors were partially supported by the National Key R\&D Program of China, Project Number 2020YFA0712000, Science Challenge Project (No. TZ2016002), and High-performance Computing Platform of Peking University. 


\section{Appendix A. 1D EC schemes}

This Appendix presents the semi-discrete 1D EC schemes. Consider the case of $d=1$ and omit the subscripts " 1 " denoting the $\xi_{1}$-direction. The system (2.8) and the GCLs (2.9) reduce to

$$
\frac{\partial \mathcal{U}}{\partial \tau}+\frac{\partial \mathcal{F}}{\partial \xi}=-\Phi^{\prime}(\boldsymbol{V})^{\mathrm{T}} \frac{\partial B_{1}}{\partial \xi}
$$

and

$$
\begin{array}{ll}
\text { VCL: } & \frac{\partial J}{\partial \tau}+\frac{\partial}{\partial \xi}\left(\frac{\partial x}{\partial t}\right)=0, \\
\text { SCL: } & \frac{\partial}{\partial \xi}\left(J \frac{\partial \xi}{\partial x}\right) \equiv 0
\end{array}
$$

where

$$
J=\frac{\partial x}{\partial \xi}, \boldsymbol{U}=J \boldsymbol{U}, \mathcal{F}=\left(J \frac{\partial \xi}{\partial t} \boldsymbol{U}\right)+\boldsymbol{F}=\left(\frac{\partial x}{\partial t} \boldsymbol{U}\right)+\boldsymbol{F}
$$

It is easy to see that the SCL holds automatically in this case. If replacing $i$ with $i$, then the $2 p$ th-order EC schemes become

$$
\begin{aligned}
\frac{\mathrm{d}}{\mathrm{d} t} \mathcal{U}_{i} & =-\frac{1}{\Delta \xi}\left(\widetilde{\mathcal{F}}_{i+\frac{1}{2}}^{2 p \mathrm{th}}-\widetilde{\mathcal{F}}_{i-\frac{1}{2}}^{2 p \mathrm{th}}\right)-\Phi^{\prime}\left(\boldsymbol{V}_{i}\right)^{\mathrm{T}} \frac{1}{\Delta \xi}\left(\left(\widetilde{B_{1}}\right)_{i+\frac{1}{2}}^{2 p \mathrm{th}}-\left(\widetilde{B_{1}}\right)_{i-\frac{1}{2}}^{2 p \mathrm{th}}\right), \\
\frac{\mathrm{d}}{\mathrm{d} t} J_{i} & =-\frac{1}{\Delta \xi}\left(\left(\frac{\widetilde{\partial x}}{\partial t}\right)_{i+\frac{1}{2}}^{2 p \mathrm{th}}-\left(\frac{\widetilde{\partial x}}{\partial t}\right)_{i-\frac{1}{2}}^{2 p \mathrm{th}}\right),
\end{aligned}
$$

where

$$
\begin{aligned}
& \widetilde{\mathcal{F}}_{i+\frac{1}{2}}^{2 p \text { th }}=\sum_{n=1}^{p} \alpha_{p, n} \sum_{s=0}^{n-1}\left[\frac{1}{2}\left(\left(\frac{\partial x}{\partial t}\right)_{i-s}+\left(\frac{\partial x}{\partial t}\right)_{i-s+n}\right) \widetilde{\boldsymbol{U}}\left(\boldsymbol{U}_{i-s}, \boldsymbol{U}_{i-s+n}\right)+\widetilde{\boldsymbol{F}}\left(\boldsymbol{U}_{i-s}, \boldsymbol{U}_{i-s+n}\right)\right] \\
& \left(\widetilde{B_{1}}\right)_{i+\frac{1}{2}}^{2 p \text { th }}=\sum_{n=1}^{p} \alpha_{p, n} \sum_{s=0}^{n-1} \frac{1}{2}\left(\left(B_{1}\right)_{i-s}+\left(B_{1}\right)_{i-s+n}\right), \\
& \left(\frac{\widetilde{\partial x}}{\partial t}\right)_{i+\frac{1}{2}}^{2 p \text { th }}=\sum_{n=1}^{p} \alpha_{p, n} \sum_{s=0}^{n-1} \frac{1}{2}\left(\left(\frac{\partial x}{\partial t}\right)_{i-s}+\left(\frac{\partial x}{\partial t}\right)_{i-s+n}\right),
\end{aligned}
$$

and $\left.\left(\frac{\partial x}{\partial t}\right)\right|_{i}$ is the mesh velocity at $\xi_{i}$.

\section{Appendix B. 2D EC schemes}

This Appendix presents the semi-discrete 2D EC schemes. Consider the case of $d=2$, and replace $\left(\xi_{1}, \xi_{2}\right)$ and $\left(x_{1}, x_{2}\right)$ with $(\xi, \eta)$ and $(x, y)$, respectively. The system (2.8) and the GCLs (2.9) reduce to

$$
\frac{\partial \mathcal{U}}{\partial \tau}+\frac{\partial \mathcal{F}_{1}}{\partial \xi}+\frac{\partial \mathcal{F}_{2}}{\partial \eta}=-\Phi^{\prime}(\boldsymbol{V})^{\mathrm{T}}\left(\frac{\partial \mathcal{B}_{1}}{\partial \xi}+\frac{\partial \mathcal{B}_{2}}{\partial \eta}\right)
$$


and

$$
\begin{aligned}
\text { VCL: } & \frac{\partial J}{\partial \tau}+\frac{\partial}{\partial \xi}\left(J \frac{\partial \xi}{\partial t}\right)+\frac{\partial}{\partial \eta}\left(J \frac{\partial \eta}{\partial t}\right)=0, \\
\text { SCLs: } & \frac{\partial}{\partial \xi}\left(J \frac{\partial \xi}{\partial x}\right)+\frac{\partial}{\partial \eta}\left(J \frac{\partial \eta}{\partial x}\right)=0 \\
& \frac{\partial}{\partial \xi}\left(J \frac{\partial \xi}{\partial y}\right)+\frac{\partial}{\partial \eta}\left(J \frac{\partial \eta}{\partial y}\right)=0
\end{aligned}
$$

where

$$
\begin{aligned}
& J=\frac{\partial x}{\partial \xi} \frac{\partial y}{\partial \eta}-\frac{\partial x}{\partial \eta} \frac{\partial y}{\partial \xi}, \boldsymbol{U}=J \boldsymbol{U} \\
& \mathcal{F}_{1}=\left(J \frac{\partial \xi}{\partial t} \boldsymbol{U}\right)+\left(J \frac{\partial \xi}{\partial x}\right) \boldsymbol{F}_{1}+\left(J \frac{\partial \xi}{\partial y}\right) \boldsymbol{F}_{2}, \mathcal{B}_{1}=\left(J \frac{\partial \xi}{\partial x}\right) B_{1}+\left(J \frac{\partial \xi}{\partial y}\right) B_{2}, \\
& \mathcal{F}_{2}=\left(J \frac{\partial \eta}{\partial t} \boldsymbol{U}\right)+\left(J \frac{\partial \eta}{\partial x}\right) \boldsymbol{F}_{1}+\left(J \frac{\partial \eta}{\partial y}\right) \boldsymbol{F}_{2}, \mathcal{B}_{2}=\left(J \frac{\partial \eta}{\partial x}\right) B_{1}+\left(J \frac{\partial \eta}{\partial y}\right) B_{2} .
\end{aligned}
$$

If replacing $i$ with $\{i, j\}$, then the $2 p$ th-order EC schemes become

$$
\begin{aligned}
\frac{\mathrm{d}}{\mathrm{d} t} \mathcal{U}_{i, j}= & -\frac{1}{\Delta \xi}\left(\left(\widetilde{\mathcal{F}_{1}}\right)_{i+\frac{1}{2}, j}^{2 p \mathrm{th}}-\left(\widetilde{\mathcal{F}_{1}}\right)_{i-\frac{1}{2}, j}^{2 p \mathrm{th}}\right)-\frac{1}{\Delta \eta}\left(\left(\widetilde{\mathcal{F}_{2}}\right)_{i, j+\frac{1}{2}}^{2 p \mathrm{th}}-\left(\widetilde{\mathcal{F}_{2}}\right)_{i, j-\frac{1}{2}}^{2 p \mathrm{th}}\right) \\
& -\Phi^{\prime}\left(\boldsymbol{V}_{i, j}\right)^{\mathrm{T}} \frac{1}{\Delta \xi}\left(\left(\widetilde{\mathcal{B}_{1}}\right)_{i+\frac{1}{2}, j}^{2 p \mathrm{th}}-\left(\widetilde{\mathcal{B}_{1}}\right)_{i-\frac{1}{2}, j}^{2 p \text { th }}\right)-\Phi^{\prime}\left(\boldsymbol{V}_{i, j}\right)^{\mathrm{T}} \frac{1}{\Delta \eta}\left(\left(\widetilde{\mathcal{B}_{2}}\right)_{i, j+\frac{1}{2}}^{2 p \text { th }}-\left(\widetilde{\mathcal{B}_{2}}\right)_{i, j-\frac{1}{2}}^{2 p \mathrm{th}}\right), \\
\frac{\mathrm{d}}{\mathrm{d} t} J_{i, j}= & -\frac{1}{\Delta \xi}\left(\left(\widetilde{\partial \frac{\partial \xi}{\partial t}}\right)_{i+\frac{1}{2}, j}^{2 p \mathrm{th}}-(\widetilde{\partial \xi})_{i-\frac{1}{2}, j}^{2 p \mathrm{th}}\right)-\frac{1}{\Delta \eta}\left(\left(\widetilde{\frac{\partial \eta}{\partial t}}\right)_{i, j+\frac{1}{2}}^{2 p \text { th }}-\left(J \frac{\partial \eta}{\partial t}\right)_{i, j-\frac{1}{2}}^{2 p \text { th }}\right),
\end{aligned}
$$

where

$$
\begin{aligned}
& \left(\widetilde{\mathcal{F}}_{1}\right)_{i+\frac{1}{2}, j}^{2 p \text { th }}=\sum_{n=1}^{p} \alpha_{p, n} \sum_{s=0}^{n-1}\left[\frac{1}{2}\left(\left(J \frac{\partial \xi}{\partial t}\right)_{i-s, j}+\left(J \frac{\partial \xi}{\partial t}\right)_{i-s+n, j}\right) \widetilde{\boldsymbol{U}}\left(\boldsymbol{U}_{i-s, j}, \boldsymbol{U}_{i-s+n, j}\right)\right. \\
& +\frac{1}{2}\left(\left(J \frac{\partial \xi}{\partial x}\right)_{i-s, j}+\left(J \frac{\partial \xi}{\partial x}\right)_{i-s+n, j}\right) \widetilde{\boldsymbol{F}}_{1}\left(\boldsymbol{U}_{i-s, j}, \boldsymbol{U}_{i-s+n, j}\right) \\
& \left.+\frac{1}{2}\left(\left(J \frac{\partial \xi}{\partial y}\right)_{i-s, j}+\left(J \frac{\partial \xi}{\partial y}\right)_{i-s+n, j}\right) \widetilde{\boldsymbol{F}_{2}}\left(\boldsymbol{U}_{i-s, j}, \boldsymbol{U}_{i-s+n, j}\right)\right], \\
& \left(\widetilde{\mathcal{F}}_{2}\right)_{i, j+\frac{1}{2}}^{2 p \text { th }}=\sum_{n=1}^{p} \alpha_{p, n} \sum_{s=0}^{n-1}\left[\frac{1}{2}\left(\left(J \frac{\partial \eta}{\partial t}\right)_{i, j-s}+\left(J \frac{\partial \eta}{\partial t}\right)_{i, j-s+n}\right) \widetilde{\boldsymbol{U}}\left(\boldsymbol{U}_{i, j-s}, \boldsymbol{U}_{i, j-s+n}\right)\right. \\
& +\frac{1}{2}\left(\left(J \frac{\partial \eta}{\partial x}\right)_{i, j-s}+\left(J \frac{\partial \eta}{\partial x}\right)_{i, j-s+n}\right) \widetilde{\boldsymbol{F}}_{1}\left(\boldsymbol{U}_{i, j-s}, \boldsymbol{U}_{i, j-s+n}\right) \\
& \left.+\frac{1}{2}\left(\left(J \frac{\partial \eta}{\partial y}\right)_{i, j-s}+\left(J \frac{\partial \eta}{\partial y}\right)_{i, j-s+n}\right) \widetilde{\boldsymbol{F}}_{2}\left(\boldsymbol{U}_{i, j-s}, \boldsymbol{U}_{i, j-s+n}\right)\right], \\
& \left(\widetilde{\mathcal{B}_{1}}\right)_{i+\frac{1}{2}, j}^{2 p t h}=\sum_{n=1}^{p} \alpha_{p, n} \sum_{s=0}^{n-1}\left[\frac{1}{4}\left(\left(J \frac{\partial \xi}{\partial x}\right)_{i-s, j}+\left(J \frac{\partial \xi}{\partial x}\right)_{i-s+n, j}\right)\left(\left(B_{1}\right)_{i-s, j}+\left(B_{1}\right)_{i-s+n, j}\right)\right.
\end{aligned}
$$




$$
\begin{aligned}
& \left.+\frac{1}{4}\left(\left(J \frac{\partial \xi}{\partial y}\right)_{i-s, j}+\left(J \frac{\partial \xi}{\partial y}\right)_{i-s+n, j}\right)\left(\left(B_{2}\right)_{i-s, j}+\left(B_{2}\right)_{i-s+n, j}\right)\right] \\
& \left(\widetilde{\mathcal{B}_{2}}\right)_{i, j+\frac{1}{2}}^{2 p \text { th }}=\sum_{n=1}^{p} \alpha_{p, n} \sum_{s=0}^{n-1}\left[\frac{1}{4}\left(\left(J \frac{\partial \eta}{\partial x}\right)_{i, j-s}+\left(J \frac{\partial \eta}{\partial x}\right)_{i, j-s+n}\right)\left(\left(B_{1}\right)_{i, j-s}+\left(B_{1}\right)_{i, j-s+n}\right)\right. \\
& \left.+\frac{1}{4}\left(\left(J \frac{\partial \eta}{\partial y}\right)_{i, j-s}+\left(J \frac{\partial \eta}{\partial y}\right)_{i, j-s+n}\right)\left(\left(B_{2}\right)_{i, j-s}+\left(B_{2}\right)_{i, j-s+n}\right)\right] \\
& (\widetilde{\partial \xi})_{i+\frac{1}{2}, j}^{2 p \text { th }}=\sum_{n=1}^{p} \alpha_{p, n} \sum_{s=0}^{n-1} \frac{1}{2}\left(\left(J \frac{\partial \xi}{\partial t}\right)_{i-s, j}+\left(J \frac{\partial \xi}{\partial t}\right)_{i-s+n, j}\right) \\
& \left(\widetilde{\left.\partial \frac{\partial \eta}{\partial t}\right)_{i, j+\frac{1}{2}}^{2 p t h}}=\sum_{n=1}^{p} \alpha_{p, n} \sum_{s=0}^{n-1} \frac{1}{2}\left(\left(J \frac{\partial \eta}{\partial t}\right)_{i, j-s}+\left(J \frac{\partial \eta}{\partial t}\right)_{i, j-s+n}\right)\right. \\
& \left(J \frac{\partial \xi}{\partial t}\right)_{i, j}=-(\dot{x})_{i, j}\left(J \frac{\partial \xi}{\partial x}\right)_{i, j}-(\dot{y})_{i, j}\left(J \frac{\partial \xi}{\partial y}\right)_{i, j}, \\
& \left(J \frac{\partial \eta}{\partial t}\right)_{i, j}=-(\dot{x})_{i, j}\left(J \frac{\partial \eta}{\partial x}\right)_{i, j}-(\dot{y})_{i, j}\left(J \frac{\partial \eta}{\partial y}\right)_{i, j} \\
& \left(J \frac{\partial \xi}{\partial x}\right)_{i, j}=+\sum_{n=1}^{p} \frac{\alpha_{p, n}}{2}\left(y_{i, j+n}-y_{i, j-n}\right) \\
& \left(J \frac{\partial \xi}{\partial y}\right)_{i, j}=-\sum_{n=1}^{p} \frac{\alpha_{p, n}}{2}\left(x_{i, j+n}-x_{i, j-n}\right) \\
& \left(J \frac{\partial \eta}{\partial x}\right)_{i, j}=-\sum_{n=1}^{p} \frac{\alpha_{p, n}}{2}\left(y_{i+n, j}-y_{i-n, j}\right) \\
& \left(J \frac{\partial \eta}{\partial y}\right)_{i, j}=+\sum_{n=1}^{p} \frac{\alpha_{p, n}}{2}\left(x_{i+n, j}-x_{i-n, j}\right) .
\end{aligned}
$$

\section{References}

[1] A.M. Anile and S. Pennisi, On the mathematical structure of test relativistic magnetofluiddynamic, Ann. Inst. Henri Poincaré, 46 (1987).

[2] L. Antón, J.A. Miralles, J.M. Martí, J.M. Ibáñez, M.A. Aloy, and P. Mimica, Relativistic magnetohydrodynamics: Renormalized eigenvectors and full wave decomposition Riemann solver, Astrophys. Journal, Suppl. Ser., 188 (2010), 1-31.

[3] D.S. Balsara and J. Kim, A subluminal relativistic magnetohydrodynamics scheme with ADER-WENO predictor and multidimensional Riemann solver-based corrector, J. Comput. Phys., 312 (2016), 357-384.

[4] D. Bhoriya and H. Kumar, Entropy-stable schemes for relativistic hydrodynamics equations, Z. Angew. Math. Phys., 71 (2020), 1-29. 
[5] B. Biswas and R.K. Dubey, Low dissipative entropy stable schemes using third order WENO and TVD reconstructions, Adv. Comput. Math., 44 (2018), 1153-1181.

[6] F. Bouchut, C. Bourdarias, and B. Perthame, A MUSCL method satisfying all the numerical entropy inequalities, Math. Comp., 65 (1996), 1439-1461.

[7] W.M. Cao, W.Z. Huang, and R.D. Russell, A study of monitor functions for two-dimensional adaptive mesh generation, SIAM J. Sci. Comput., 20 (1999), 1978-1994.

[8] M.H. Carpenter, T.C. Fisher, E.J. Nielsen, and S.H. Frankel, Entropy stable spectral collocation schemes for the Navier-Stokes equations: Discontinuous interfaces, SIAM J. Sci. Comput., 36 (2014), B835-B867.

[9] J. Chan, On discretely entropy conservative and entropy stable discontinuous Galerkin methods, J. Comput. Phys., 362 (2018), 346-374.

[10] T.H. Chen and C.W. Shu, Entropy stable high order discontinuous Galerkin methods with suitable quadrature rules for hyperbolic conservation, J. Comput. Phys., 345 (2017), 427-461.

[11] T.H. Chen and C.W. Shu, Review of entropy stable discontinuous Galerkin methods for systems of conservation laws on unstructured simplex meshes, CSIAM Trans. Appl. Math., 1 (2020), $1-52$.

[12] Y.P. Chen, Y.Y. Kuang, and H.Z. Tang, Second-order accurate genuine BGK schemes for the ultra-relativistic flow simulations, J. Comput. Phys., 349 (2017), 300-327.

[13] Y.P. Chen, Y.Y. Kuang, and H.Z. Tang, Second-order accurate BGK schemes for the special relativistic hydrodynamics with the synge equation of state, J. Comput. Phys., 442 (2021), 110438.

[14] M.G. Crandall and A. Majda, Monotone difference approximations for scalar conservation laws, Math. Comp., 34 (1980), 1-21.

[15] L. Del Zanna, N. Bucciantini, and P. Londrillo, An efficient shock-capturing central-type scheme for multi-dimensional relativistic flows, I: Hydrodynamics, Astron. Astrophys., 3 (2002), 1177-1186.

[16] L. Del Zanna, N. Bucciantini, and P. Londrillo, An efficient shock-capturing central-type scheme for multidimensional relativistic flows, II. Magnetohydrodynamics, Astron. Astrophys., 400 (2003), 397-413. 
[17] A. Dolezal and S.S.M. Wong, Relativistic hydrodynamics and essentially non-oscillatory shock capturing schemes, J. Comput. Phys., 120 (1995), 266-277.

[18] J. Duan and H. Tang, An analytical solution of the isentropic vortex problem in the special relativistic magnetohydrodynamics, arXiv 210\%.01966, (2021).

[19] J.M. Duan and H.Z. Tang, High-order accurate entropy stable finite difference schemes for oneand two-dimensional special relativistic hydrodynamics, Adv. Appl. Math. Mech., 12 (2020), $1-29$.

[20] J.M. Duan and H.Z. Tang, High-order accurate entropy stable nodal discontinuous Galerkin schemes for the ideal special relativistic magnetohydrodynamics, J. Comput. Phys., 421 (2020), 109731.

[21] J.M. Duan and H.Z. Tang, Entropy stable adaptive moving mesh schemes for 2D and 3D special relativistic hydrodynamics, J. Comput. Phys., 426 (2021), 109949.

[22] F. Eulderink and G. Mellema, General relativistic hydrodynamics with a Roe solver, Astron. Astrophys. Suppl. Ser., 110 (1994), 34.

[23] T.C. Fisher and M.H. Carpenter, High-order entropy stable finite difference schemes for nonlinear conservation laws: Finite domains, J. Comput. Phys., 252 (2013), 518-557.

[24] U.S. Fjordholm, S. Mishra, and E. Tadmor, Arbitrarily high-order accurate entropy stable essentially non-oscillatory schemes for systems of conservation laws, SIAM J. Numer. Anal., 50 (2012), 544-573.

[25] J.A. Font, Numerical hydrodynamics and magnetohydrodynamics in general relativity, Living Rev. Relativ., 11 (2008), 7.

[26] G.J. Gassner, A skew-symmetric discontinuous Galerkin spectral element discretization and its relation to SBP-SAT finite difference methods, SIAM J. Sci. Comput., 35 (2013), 1233-1253.

[27] G.J. Gassner and A.R. Winters, A novel robust strategy for discontinuous Galerkin methods in computational fluid mechanics: Why? When? What? Where?, Front. Phys., 8 (2021), 500690.

[28] S.K. Godunov, Symmetric form of the equations of magnetohydrodynamics, Numer. Meth. Mech. Cont. Medium, 1 (1972), 26-34.

[29] J.Q. Han and H.Z. Tang, An adaptive moving mesh method for two-dimensional ideal magnetohydrodynamics, J. Comput. Phys., 220 (2007), 791-812. 
[30] A. Harten, J.M. Hyman, and P.D. Lax, On finite-difference approximations and entropy conditions for shocks, Comm. Pure Appl. Math., 29 (1976), 297-322.

[31] P. He and H.Z. Tang, An adaptive moving mesh method for two-dimensional relativistic hydrodynamics, Commun. Comput. Phys., 11 (2012), 114-146.

[32] P. He and H.Z. Tang, An adaptive moving mesh method for two-dimensional relativistic magnetohydrodynamics, Comput. Fluids, 60 (2012), 1-20.

[33] A. Hiltebrand and S. Mishra, Entropy stable shock capturing space-time discontinuous Galerkin schemes for systems of conservation laws, Numer. Math., 126 (2014), 103-151.

[34] T.J. Hughes, L. Franca, and M. Mallet, A new finite element formulation for computational fluid dynamics: I. Symmetric forms of the compressible Euler and Navier-Stokes equations and the second law of thermodynamics, Comput. Methods Appl. Mech. Engrg., 54 (1986), 223-234.

[35] F. Ismail and P.L. Roe, Affordable, entropy-consistent Euler flux functions II: Entropy production at shocks, J. Comput. Phys., 228 (2009), 5410-5436.

[36] G.S. Jiang and C.W. Shu, Efficient implementation of weighted ENO schemes, J. Comput. Phys., 126 (1996), 202-228.

[37] A.V. Koldoba, O.A. Kuznetsov, and G.V. Ustyugova, An approximate Riemann solver for relativistic magnetohydrodynamics, Mon. Not. R. Astron. Soc., 333 (2002), 932-942.

[38] P.G. LeFloch, J.M. Mercier, and C. Rohde, Fully discrete entropy conservative schemes of arbitraty order, SIAM J. Numer. Anal., 40 (2002), 1968-1992.

[39] D. Ling, J.M. Duan, and H.Z. Tang, Physical-constraints-preserving Lagrangian finite volume schemes for one- and two-dimensional special relativistic hydrodynamics, J. Comput. Phys., 396 (2019), 507-543.

[40] D. Ling and H.Z. Tang, A physical-constraints-preserving genuinely multidimensional hll scheme for the special relativistic hydrodynamics, arXiv: 2011.00906, (2020).

[41] J.M. Martí and E. Müller, Extension of the piecewise parabolic method to one-dimensional relativistic hydrodynamics, J. Comput. Phys., 123 (1996), 1-14.

[42] J.M. Martí and E. Müller, Numerical hydrodynamics in special relativity, Living Rev. Relativ., 6 (2003), 7. 
[43] J.M. Martí and E. Müller, Grid-based methods in relativistic hydrodynamics and magnetohydrodynamics, Living Rev. Comput. Astrophys., 1 (2015), 3.

[44] M.M. May and R.H. White, Hydrodynamic calculations of general-relativistic collapse, Phys. Rev., 141 (1966), 1232-1241.

[45] M.M. May and R.H. White, Stellar dynamics and gravitational collapse, in B. Alder, S. Fernbach, and M. Rotenberg, editors, Methods Comput. Phys., vol. 7, New York: Academic (1967), $219-258$.

[46] A. Mignone and G. Bodo, An HLLC Riemman solver for relativistic flows - I. Hydrodynamics, Mon. Not. R. Astron. Soc., 136 (2005), 126-136.

[47] A. Mignone and G. Bodo, An HLLC Riemann solver for relativistic flows - II. Magnetohydrodynamics, Mon. Not. R. Astron. Soc., 368 (2006), 1040-1054.

[48] A. Mignone, G. Bodo, S. Massaglia, T. Matsakos, O. Tesileanu, C. Zanni, and A. Ferrari, PLUTO: A numerical code for computational astrophysics, Astrophys. J. Suppl. Ser., 170 (2007), 228-242.

[49] A. Mignone, T. Plewa, and G. Bodo, The piecewise parabolic method for multidimensional relativistic fluid dynamics, Astron. Astrophys. Suppl. Ser., 160 (2005), 199-219.

[50] A. Mignone, M. Ugliano, and G. Bodo, A five-wave Harten-Lax-van Leer Riemann solver for relativistic magnetohydrodynamics, Mon. Not. R. Astron. Soc., 393 (2009), 1141-1156.

[51] S. Osher, Riemann solvers, the entropy condition, and difference approximations, SIAM J. Numer. Anal., 21 (1984), 217-235.

[52] S. Osher and E. Tadmor, On the convergence of difference approximations to scalar conservation laws, Math. Comp., 50 (1988), 19-51.

[53] K.G. Powell, An approximate riemann solver for magnetohydrodynamics (that works in more than one dimension), ICASE 94-24, (1994).

[54] L. Rezzolla and O. Zanotti, Relativistic Hydrodynamics, Oxford University Press (2013).

[55] V. Schneider, U. Katscher, D.H. Rischke, B. Waldhauser, J.A. Maruhn, and C.D. Munz, New algorithms for ultra-relativistic numerical hydrodynamics, J. Comput. Phys., 105 (1993), 92107. 
[56] E. Tadmor, The numerical viscosity of entropy stable schemes for systems of conservation laws, I, Math. Comp., 49 (1987), 91-103.

[57] E. Tadmor, Entropy stability theory for difference approximations of nonlinear conservation laws and related time-dependent problems, Acta Numer., 12 (2003), 451-512.

[58] H.Z. Tang, A moving mesh method for the Euler flow calculations using a directional monitor function, Commun. Comput. Phys., 1 (2006), 656-676.

[59] H.Z. Tang, T. Tang, and P.W. Zhang, An adaptive mesh redistribution method for nonlinear Hamilton-Jacobi equations in two- and three-dimensions, J. Comput. Phys., 188 (2003), 543572 .

[60] P.D. Thomas and C.K. Lombard, Geometric conservation law and its application to flow computations on moving grids, AIAA J., 17 (1979), 1030-1037.

[61] J.R. Wilson, Numerical study of fluid flow in a kerr space, Astrophys. J., 173 (1972), 431-438.

[62] A.M. Winslow, Numerical solution of the quasilinear Poisson equation in a nonuniform triangle mesh, J. Comput. Phys., 1 (1967), 149-172.

[63] K.L. Wu, Design of provably physical-constraint-preserving methods for general relativistic hydrodynamics, Phys. Rev. D, 95 (2017), 103001.

[64] K.L. Wu and C.W. Shu, Entropy symmetrization and high-order accurate entropy stable numerical schemes for relativistic MHD equations, SIAM J. Sci. Comput., 42 (2020), A2230A2261.

[65] K.L. Wu and H.Z. Tang, High-order accurate physical-constraints-preserving finite difference WENO schemes for special relativistic hydrodynamics, J. Comput. Phys., 298 (2015), 539-564.

[66] K.L. Wu and H.Z. Tang, A direct Eulerian GRP scheme for spherically symmetric general relativistic hydrodynamics, SIAM J. Sci. Comput., 38 (2016), B458-B489.

[67] K.L. Wu and H.Z. Tang, Physical-constraint-preserving central discontinuous Galerkin methods for special relativistic hydrodynamics with a general equation of state, Astrophys. J. Suppl. Ser., 228 (2016), 3.

[68] K.L. Wu and H.Z. Tang, Admissible state and physical-constraints-preserving schemes for relativistic magnetohydrodynamic equations, Math. Models Methods Appl. Sci., 27 (2017), 1871-1928. 
[69] K.L. Wu and H.Z. Tang, On physical-constraints-preserving schemes for special relativistic magnetohydrodynamics with a general equation of state, Z. Angew. Math. Phys., 69 (2018), $1-24$.

[70] K.L. Wu, Z.C. Yang, and H.Z. Tang, A third-order accurate direct Eulerian GRP scheme for one-dimensional relativistic hydrodynamics, East Asian J. Appl. Math., 4 (2014), 95-131.

[71] Z.C. Yang, P. He, and H.Z. Tang, A direct Eulerian GRP scheme for relativistic hydrodynamics: One-dimensional case, J. Comput. Phys., 230 (2011), 7964-7987.

[72] Z.C. Yang and H.Z. Tang, A direct Eulerian GRP scheme for relativistic hydrodynamics: Two-dimensional case, J. Comput. Phys., 231 (2012), 2116-2139.

[73] Y.H. Yuan and H.Z. Tang, Two-stage fourth-order accurate time discretizations for 1D and 2D special relativistic hydrodynamics, J. Comput. Math., 38 (2020), 768-796.

[74] H. Zhang, M. Reggio, J.Y. Trépanier, and R. Camarero, Discrete form of the GCL for moving meshes and its implementation in CFD schemes, Comput. ES Fluids, 22 (1993), 9-23.

[75] J. Zhao and H.Z. Tang, Runge-Kutta discontinuous Galerkin methods with WENO limiter for the special relativistic hydrodynamics, J. Comput. Phys., 242 (2013), 138-168.

[76] J. Zhao and H.Z. Tang, Runge-Kutta discontinuous Galerkin methods for the special relativistic magnetohydrodynamics, J. Comput. Phys., 343 (2017), 33-72. 